EDITORIAL BOARD

ANTONIO CARCATERRA

ERIC A. CARLEN

FRANCESCO DELL'ISOLA

RAFFAELE ESPOSITO

ALBERT FANNJIANG

Gilles A. FranCFORT

PiERANGELO MARCATI

JEAN-JACQUES MARIGO

PETER A. MARKOWICH

MARTIN OSTOJA-STARZEWSKI

PIERRE SEPPECHER

DAVID J. STEIGMANN

PAUl STEINMANN

PierRe M. SuQueT

MANAGING EDITORS

MICOL AMAR

EMILIO BARCHIESI

ANGELA MADEO

MARTIN OSTOJA-STARZEWSKI

ADVISORY BOARD

ADNAN AKAY

Holm AltenbaCH

MICOL AMAR

HARM ASKES

TEODOR ATANACKOVIĆ

VICTOR BERDICHEVSKY

GUY BOUCHITTÉ

ANDREA BRAIDES

ROBERTO CAMASSA

MAURO CARFORE

ERIC DARVE

FELIX DARVE

ANNA DE MASI

Gianpietro Del Piero

EMMANUELE Di BENEDETTO

VICTOR A. EREMEYEV

BERNOLD FIEDLER

IRENE M. GAMBA

DAVID Y. GAO

Sergey GaVRILyuk

Timothy J. HEALEY

DOMINIQUe JEULiN

ROGER E. KHAYAT

Corrado LATTANZIO

ROBERT P. LIPTON

ANGELO LUONGO

ANGELA MADEO

JUAN J. MANFREDI

CARLO MARCHIORO ANIL MisRa

ROBERTO NATALINI PATRIZIO NEFF

Thomas J. PENCE

ANDREY PIATNITSKI

ERRICO PRESUTTI

MARIO Pulvirenti

LUCIO RUSSO

Miguel A. F. SAnjuaN

PATRICK SElVAdurai

MiroslaV ŠILHAVÝ

GUIDO SWEERS

ANTOINETTE TORDESILLAS

LEV TRUSKINOVSKY

JUAN J. L. VELÁZQUEZ

VINCENZO VESPRI

ANGELO VULPIANI msp.org/memocs

Università di Roma "La Sapienza", Italia

Rutgers University, USA

(CO-CHAIR) Università di Roma "La Sapienza", Italia

(TREASURER) Università dell'Aquila, Italia

University of California at Davis, USA

(CO-CHAIR) Université Paris-Nord, France

Università dell' Aquila, Italy

École Polytechnique, France

DAMTP Cambridge, UK, and University of Vienna, Austria

(CHAIR MANAGING EDITOR) Univ. of Illinois at Urbana-Champaign, USA

Université du Sud Toulon-Var, France

University of California at Berkeley, USA

Universität Erlangen-Nürnberg, Germany

LMA CNRS Marseille, France

Università di Roma "La Sapienza”, Italia

Università degli Studi dell'Aquila, Italy

Université de Lyon-INSA (Institut National des Sciences Appliquées), France

(CHAIR MANAGING EDITOR) Univ. of Illinois at Urbana-Champaign, USA

Carnegie Mellon University, USA, and Bilkent University, Turkey

Otto-von-Guericke-Universität Magdeburg, Germany

Università di Roma "La Sapienza", Italia

University of Sheffield, UK

University of Novi Sad, Serbia

Wayne State University, USA

Université du Sud Toulon-Var, France

Università di Roma Tor Vergata, Italia

University of North Carolina at Chapel Hill, USA

Università di Pavia, Italia

Stanford University, USA

Institut Polytechnique de Grenoble, France

Università dell'Aquila, Italia

Università di Ferrara and International Research Center MEMOCS, Italia

Vanderbilt University, USA

Gdansk University of Technology, Poland

Freie Universität Berlin, Germany

University of Texas at Austin, USA

Federation University and Australian National University, Australia

Université Aix-Marseille, France

Cornell University, USA

École des Mines, France

University of Western Ontario, Canada

Università dell' Aquila, Italy

Louisiana State University, USA

Università dell'Aquila, Italia

Université de Lyon-INSA (Institut National des Sciences Appliquées), France

University of Pittsburgh, USA

Università di Roma "La Sapienza", Italia

University of Kansas, USA

Istituto per le Applicazioni del Calcolo "M. Picone", Italy

Universität Duisburg-Essen, Germany

Michigan State University, USA

Narvik University College, Norway, Russia

Università di Roma Tor Vergata, Italy

Università di Roma "La Sapienza", Italia

Università di Roma "Tor Vergata", Italia

Universidad Rey Juan Carlos, Madrid, Spain

McGill University, Canada

Academy of Sciences of the Czech Republic

Universität zu Köln, Germany

University of Melbourne, Australia

École Polytechnique, France

Bonn University, Germany

Università di Firenze, Italia

Università di Roma La Sapienza, Italia

MEMOCS (ISSN 2325-3444 electronic, 2326-7186 printed) is a journal of the International Research Center for the Mathematics and Mechanics of Complex Systems at the Università dell'Aquila, Italy.

Cover image: "Tangle” by @ John Horigan; produced using the Context Free program (contextfreeart.org).

\section{PUBLISHED BY}

\section{I. mathematical sciences publishers} nonprofit scientific publishing http://msp.org/ 


\title{
GENOTYPE-DEPENDENT VIRUS DISTRIBUTION AND COMPETITION OF VIRUS STRAINS
}

\author{
Nikolai Bessonov, Gennady A. Bocharov, \\ CRISTINA LEON, Vladimir POPOV AND Vitaly VolPERT
}

\begin{abstract}
Virus density distribution as a function of genotype considered as a continuous variable and of time is studied with a nonlocal reaction-diffusion equation taking into account virus competition for the host cells and its elimination by the immune response and by the genotype-dependent mortality. The existence of virus strains, that is, of positive stable stationary solutions decaying at infinity, is determined by the admissible intervals in the genotype space where the genotype-dependent mortality is less than the virus reproduction rate, and by the immune response under some appropriate assumptions on the immune response function characterizing virus elimination by immune cells. The competition of virus strains is studied, first, without immune response and then with the immune response. In the absence of immune response, the strain dynamics is different in a short time scale where they converge to some intermediate slowly evolving solutions depending on the initial conditions, and in a long time scale where their distribution converges to a stationary solution. Immune response can essentially influence the strain dynamics either stabilizing them or eliminating one of the strains. An antiviral treatment can also influence the competition of virus strains, and it can lead to the emergence of resistant strains, which were absent before the treatment because of the competition with susceptible strains.
\end{abstract}

\section{Introduction}

A fundamental feature of many RNA virus infections of major public concern (e.g., human immunodeficiency virus type I (HIV) and hepatitis C virus (HCV)) is an error-prone replication [Domingo and Perales 2018]. The high genetic variability of HIV and HCV and selection of the most adapted mutants determine the ability of the virus population to escape the immune response and develop resistance to the antiviral therapy [Coffin and Swanstrom 2013; Gaudieri et al. 2009]. The variability of the viruses is considered to be one of the key factors in the pathogenesis of the respective infectious disease. Variation of the genetic structure of

Communicated by Francesco dell'Isola.

MSC2010: 35K57, 92C30.

Keywords: virus density distribution, genotype, nonlocal interaction, competition of strains. 
the viral population is the result of the interaction of the replication, mutation, recombination, immune-mediated elimination, and drug-dependent suppression of the virus replication. An important step towards a mechanistic understanding of the evolution of the heterogeneous virus populations is provided by the models which consider explicitly the infection of target cells, interaction with the immune system, and drug-dependent blockade of the virus replication.

To describe and analyze the dynamics of genetic heterogeneity of evolving virus populations, the concept of quasispecies provides a general framework to deal with an ensemble of genomes [Eigen 1971; Biebricher and Eigen 2006]. A more formal approach to studying the evolution of quasispecies is based on considering the mutation-selection processes acting on the virus strains according to their fitness values. The respective deterministic models are formulated using systems of ODEs. The standard form of the quasispecies model in mathematical virology is the set of ODE equations [Nowak and May 2000]

$$
\frac{d \boldsymbol{v}}{d t}=\boldsymbol{W} \boldsymbol{v}-d(\boldsymbol{v}) \boldsymbol{v}, \quad \boldsymbol{W}=\left(\begin{array}{cccc}
a_{1} Q_{11} & a_{2} Q_{21} & \cdots & a_{n} Q_{n 1} \\
a_{1} Q_{12} & a_{2} Q_{22} & \cdots & a_{n} Q_{n 2} \\
\vdots & \vdots & \ddots & \vdots \\
a_{1} Q_{1 n} & a_{2} Q_{2 n} & \cdots & a_{n} Q_{n n}
\end{array}\right), d(\boldsymbol{v})=\frac{\sum_{i=1}^{n} a_{i} v_{i}}{\sum_{i=1}^{n} v_{i}}
$$

Here, the vector $v$ characterizes the abundance of genomes composing the population, $\boldsymbol{v}=\left\{v_{1}, v_{1}, \ldots, v_{n}\right\}, a_{i}$ stand for the replication rates of $i$-th genome (quasispecies), $i=1, \ldots, n$, and $\boldsymbol{Q}=\left(Q_{i j}\right)$ is the mutation matrix. The last term describes the competition of the genomes for survival. The model considers the balance of production and elimination of the quasispecies and as such bears no specific link to real processes underpinning the collective dynamics of the genomes. Another framework is provided by stochastic models taking the form of genetic algorithms [Bocharov et al. 2005; Vijay et al. 2008]. The distributed parameter approach with respect to the mutant frequency as a continuous variable was proposed in [Rouzine et al. 2001] using the forward Kolmogorov equation.

Some general regularities underlying the evolution of viral quasispecies (equivalently, the ensembles of virus strains) have been elucidated empirically. The viruses can escape immune control by generating mutations within the peptide epitopes, and the epitope inducing the strongest $\mathrm{T}$ cell response is subject to the strongest selective pressure [McMichael and Carrington 2019]. The dynamics of drug-resistant mutants depends on a number of virus replication parameters, such as the availability and the spectrum of target cells, the epistatic interactions between specific mutations, etc. [Martínez et al. 2011]. However, a deeper insight into the impact of virus population properties and its sensitivity to drugs and the immune responses requires the development of mathematical models with an explicit description of 
the interplay between the above processes in producing the survival advantages of specific virus strains, characterized in general as the fitness values.

The fitness value can be estimated in vitro under certain special conditions [Martínez et al. 2011]. However, its quantification for real infections remains a challenge [Ganusov et al. 2011] as it results from a complex system of factors, such as virus production in target cells, host-dependent immune responses, and drug efficacy. We have recently developed a novel mathematical framework for predicting and quantifying the virus diversity evolution during infection of a host organism [Bessonov et al. 2020].

In this study we examine the properties of the formulated mathematical model to shed new light on the collective behavior of virus genome ensembles (strains) in relation to the parameters of mutation, replication, interaction with the immune system, and the susceptibility to the antiviral drugs. We consider the equation

$$
\frac{\partial u}{\partial t}=D \frac{\partial^{2} u}{\partial x^{2}}+a u(1-b I(u))-u f(u)-\sigma(x) u,
$$

describing the evolution of virus density depending on the genotype $x$ considered as a continuous variable and on time $t$. The first term in the right-hand side of this equation characterizes virus mutation and the second term its reproduction; the next term specifies virus elimination by immune response, and the last term its death. We now describe each of these terms in more detail.

- Assuming there is a sequence of reversible mutations with consecutive genotypes $x_{i}$, we can write the equation for the density $u_{i}$ of virus with genotype $x_{i}$ :

$$
\frac{d u_{i}}{d t}=\mu\left(u_{i-1}-u_{i}\right)+\mu\left(u_{i+1}-u_{i}\right)
$$

where $\mu$ is the frequency of mutations. This equation represents a discretization of the diffusion equation with the diffusion coefficient proportional to $\mu$. Virus mutation described by the diffusion operator was previously considered in [Kimura 1964; Sasaki 1994]. In a more general case, one should take into account a more complex mutation pattern (see, e.g., [Martínez et al. 2011]).

- The virus multiplication term is proportional to the virus density $u$ and to the quantity of uninfected host cells $(1-b I(u))$. Here 1 is a dimensionless total number of cells, and $b I(u)$ is the number of infected cells, which is proportional to the total virus quantity $I(u)=\int_{-\infty}^{\infty} u(x, t) d x$ (see Section 4 for more detail).

- Virus elimination by immune cells is proportional to the virus density and to the quantity of immune cells $c$. The latter is supposed to be a function of virus density. The function $f(u)$ is sufficiently smooth with $f(u)>0$ for $u>0$. It grows for sufficiently small $u$, since the immune response is stimulated by 
antigens, and it can be down-regulated for sufficiently large $u$, since the high viral load infection can suppress immune response (via exhaustion mechanisms). This term can contain time delay taking into account clonal expansion of immune cells [Bocharov et al. 2018], but we do not consider it in this work.

- The last term in the right-hand side of (1-2) describes virus natural death and its elimination by some antiviral treatment. Let us note that the death rate can depend on virus genotype $x$.

We consider virus strain as density distribution concentrated around some genotype value. Mathematically speaking, it is a positive solution of (1-2) decaying at infinity. We will determine conditions of the existence of such solutions to delineate the rules characterizing the competition of different strains and their response to treatment. In particular, we will see how the elimination of some strains by treatment can lead to the emergence of the strains resistant to treatment. We will begin the analysis of the existence and competition of virus strains due to the genotypedependent mortality in the absence of immune response (Section 2), and we will continue with the investigation of the influence of immune response (Section 3). We discuss the modeling approach and the results in Section 4. Some technical calculations and proofs are placed in Appendices A, B, and C in order to simplify the reading of the paper.

\section{Localized solutions in the absence of immune response}

We begin the analysis of (1-2) in the case without immune response, $f(u) \equiv 0$. We will present conditions on the death function $\sigma(x)$ providing the existence of localized positive solutions describing virus strains. After that, we will study the competition of two strains.

\section{A. Existence of stationary solutions.}

Model problem. Consider the equation

$$
D u^{\prime \prime}+u(1-I(u))-\sigma(x) u=0
$$

on the whole axis, where $I(u)=\int_{-\infty}^{\infty} u(x) d x, \sigma(x)=\sigma_{0}>1$ for $|x| \geq x_{0}$, and $\sigma(x)=0$ for $|x|<x_{0}$, for $x_{0}$ some positive number. We look for a positive bounded solution of this equation. Clearly, it can exist only if $I(u)<1$. Set

$$
1-I(u)=k^{2} .
$$

Then (2-1) can be written as

$$
D u^{\prime \prime}+k^{2} u=0, \quad|x|<x_{0}, \quad D u^{\prime \prime}+k^{2} u-\sigma_{0} u=0, \quad|x| \geq x_{0} .
$$


Therefore,

$$
u(x)=c_{1} \cos (\mu x), \quad|x|<x_{0}, \quad u(x)=c_{2} e^{ \pm \lambda x}, \quad|x| \geq x_{0},
$$

where $c_{1}$ and $c_{2}$ are some positive constants, $\mu=k / \sqrt{D}$, and $\lambda=\sqrt{\sigma_{0}-k^{2}} / \sqrt{D}$ $\left(k^{2}<\sigma_{0}\right)$. From the continuity of the solution and of its first derivative at $x= \pm x_{0}$ we obtain the equalities

$$
c_{1} \cos \left(\mu x_{0}\right)=c_{2} e^{-\lambda x_{0}}, \quad c_{1} \mu \sin \left(\mu x_{0}\right)=c_{2} \lambda e^{-\lambda x_{0}} .
$$

Dividing the second equation by the first, we get the equation with respect to $k$ :

$$
\sqrt{\sigma_{0}-k^{2}}=k \tan \left(k x_{0} / \sqrt{D}\right) .
$$

We can now determine the integral $I(u)$ :

$$
I(u)=\int_{-\infty}^{\infty} u(x) d x=\frac{2 c_{1}}{\mu} \sin \left(\mu x_{0}\right)+\frac{2 c_{2}}{\lambda} e^{-\lambda x_{0}} .
$$

Taking into account the first relation in (2-4), we have

$$
I(u)=2 c_{1}\left(\frac{1}{\mu} \sin \left(\mu x_{0}\right)+\frac{1}{\lambda} \cos \left(\mu x_{0}\right)\right) .
$$

The coefficient $c_{1}$ can be determined from (2-2):

$$
c_{1}=\left(1-k^{2}\right) /(2 h(k)), \quad h(k)=\frac{1}{\mu} \sin \left(\mu x_{0}\right)+\frac{1}{\lambda} \cos \left(\mu x_{0}\right),
$$

and $c_{2}=c_{1} e^{\lambda x_{0}} \cos \left(\mu x_{0}\right)$.

Let us recall that we are looking for a solution $k<1$ of (2-5). Such solution exists if $x_{0} / \sqrt{D}$ is greater than the critical value

$$
\xi^{*}=\frac{1}{k} \arctan \sqrt{\frac{\sigma_{0}}{k^{2}}-1},
$$

and it does not exist if $x_{0} / \sqrt{D}<\xi^{*}$. For $x_{0}$ large enough, there are multiple solutions satisfying this condition. We can now formulate the following result.

Theorem 2.1. Let $\sigma(x)=\sigma_{0}>1$ for $|x| \geq x_{0}$, and $\sigma(x)=0$ for $|x|<x_{0}$, where $x_{0}$ is some positive number. Then (2-1) has a positive solution decaying at infinity for $x_{0} / \sqrt{D}>\xi^{*}$, and such solution does not exist for $x_{0} / \sqrt{D} \leq \xi^{*}$. Here $\xi^{*}$ is given by expression (2-6).

Generalization of the existence result. The previous theorem is based on the explicit construction of a solution for a piecewise-constant function $\sigma(x)$. The existence result can be generalized for some class of functions using a more sophisticated mathematical method based on the topological degree and a priori estimates of solutions (Leray-Schauder method). 

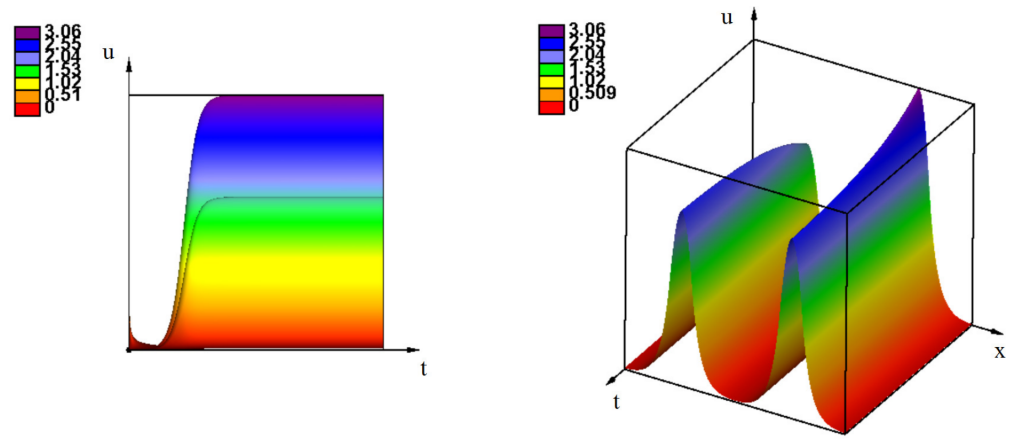

Figure 1. Solution $u(x, t)$ of (1-2) in numerical simulations. Left: projection of solution on the $(t, u)$-plane for $t=60$. Right: 3D solution for $t=10^{5}$. The values of parameters are $L=1, a=b=1$, $D=0.001, \sigma(x)=0$ for $0.2<x<0.3$ and $0.7<x<0.8$ and $=1$ otherwise, and initial condition $=0.1$ for $0.5<x<0.52$.

Theorem 2.2. Suppose that $\sigma(x)$ is a sufficiently smooth bounded function such that $\sigma(x)=0$ for $|x| \leq x_{0}$ and $\sigma(x) \geq \sigma_{0} \geq 1$ for $|x| \geq x_{1}$, where $x_{1}>x_{0}>\pi / 2$. Then (2-1) has a positive solution decaying at infinity.

The proof of this theorem is given in Appendix C.

2B. Two admissible intervals. We showed in the previous subsection that a localized positive solution of (2-1) exists for a sufficiently large admissible interval or for a small mutation rate (diffusion coefficient). This localized solution corresponds to a virus strain. In order to study the competition of two strains for the host cells, we will now consider the death rate function $\sigma(x)$ with two admissible intervals, $\sigma(x)=0$ for $x_{1} \leq|x| \leq x_{2}$ and $\sigma=\sigma_{0}>0$ otherwise. Here $x_{2}>x_{1}>0$.

The analytical solution of (1-2) with such function $\sigma(x)$ is quite complex, and it is presented in Appendix A. It is shown that existence and multiplicity of solutions can be formulated in terms of the parameter $h=\left(x_{2}-x_{1}\right) / \sqrt{D}$ characterizing the length of the admissible interval normalized by the diffusion coefficient. There exists a positive solution decaying at infinity if $h>h_{c}$ for some critical value $h_{c}$, and such solution does not exist if $h<h_{c}$. Moreover, it is shown that there are two branches of solutions; one of them is a symmetric (even) function, while another one is asymmetric. In order to study the stability of these solutions, we carry out numerical simulations of the initial boundary value problem for (1-2) on a bounded interval $0<x<L$ with periodic boundary conditions. We set $f(u) \equiv 0$, and $I(u)=\int_{0}^{L} u(x, t) d x$.

An example of numerical simulations is shown in Figures 1 and 2. Behavior of solutions is characterized by a fast convergence to an intermediate solution and 

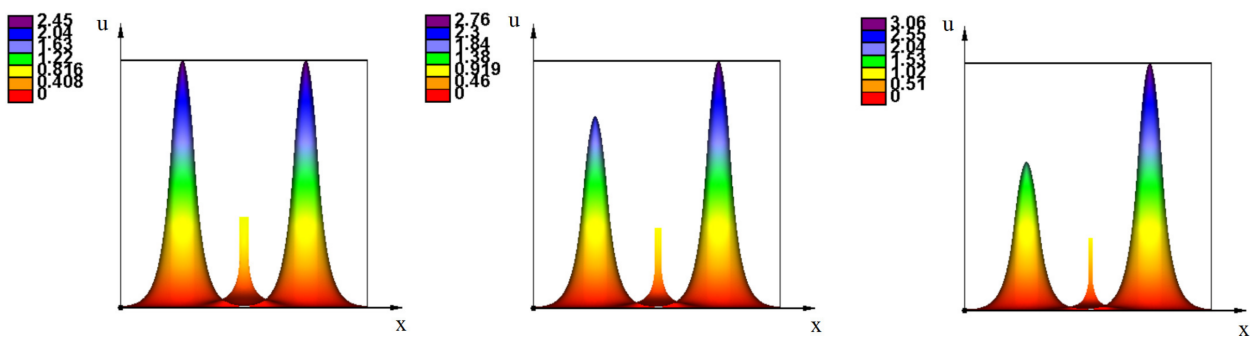

Figure 2. Solution $u(x, t)$ of (1-2) in numerical simulations. The intermediate stationary solution (projection on the $(x, u)$-plane) is shown for different initial conditions equal to 0.1 for $0.48<x<52$ (left), $0.49<x<0.52$ (middle), and $0.50<x<0.52$ (right). A small peak at the center of the interval shows the initial condition. The values of parameters are $L=1, a=b=1, D=0.001$, and $\sigma(x)=0$ for $0.2<x<0.3$ and $0.7<x<0.8$ and $=1$ otherwise.

then by a slow convergence to a stationary solution (Figure 1). The intermediate solution resembles two pulses with the maxima located at the centers of the admissible intervals. Since the initial condition is not symmetric with respect to the center of the interval, this solution is not symmetric either, and the ratio between the pulses' maxima depends on the initial condition (Figure 2). The characteristic time $T_{1}$ of the convergence to this solution is on the order of 10 (dimensionless units). After reaching their intermediate values, in this time scale they remain constant. The intermediate solution converges to the stationary solution in a longer time scale determined by the value of the diffusion coefficient (Figure 1, right). For $D=0.001$ considered in this example, it is of the order $10^{5}$, that is, four orders of magnitude larger. The stationary solution resembles two pulses symmetric with respect to the center of the interval. Thus, in terms of dynamical systems, we have a fast manifold with convergence to the intermediate solution and a slow manifold with convergence to the stationary solution. Though the stationary solution is globally asymptotically stable, dynamics of solutions in the realistic time scale can be determined by the intermediate solution.

The convergence time exponentially grows with the decrease of $D$ and becomes so large for $D<0.001$ that the stationary solution may not be reached (Appendix A, Figure 9). In this case, the dynamics of the solution is determined by the intermediate solution, which depends on the initial condition and on the parameters.

Thus, we have an unusual and counterintuitive situation where instead of a unique (for given parameters) globally stable stationary solution, we should consider a continuous family of intermediate solutions. We will discuss below biological implications of this result. 


\section{The influence of immune response}

3A. Virus reproduction and the effect of antiviral immune response. In order to study the influence of immune response on virus distribution in the space of genotypes, we begin with the case without natural genotype-dependent virus death, $\sigma(x) \equiv 0$. In this case, the equation for the virus density distribution is

$$
\frac{\partial u}{\partial t}=D \frac{\partial^{2} u}{\partial x^{2}}+a u(1-b I(u))-u f(u) .
$$

The immune response function is increasing for $u$ sufficiently small and decreasing for $u$ sufficiently large. We begin with some model examples.

Constant immune response. Set $f(u) \equiv c$, where $c$ is a positive constant. Integrating (3-1), we get the equation with respect to integral $I(u)(t)$ considered as a function of time:

$$
\frac{d I}{d t}=a I(1-c / a-b I) .
$$

If $c \geq a$, then $I(t) \rightarrow 0$ as $t \rightarrow \infty$. If $c<a$, then $I(t) \rightarrow(1-c / a) / b$. In both cases, $\sup _{x} u(x) \rightarrow 0$ as $t \rightarrow \infty$. Let us discuss this convergence in the case where $c<a$. The stationary solution $u=0$ of (3-1) is unstable in this case. Indeed, the corresponding spectral problem has a part of the spectrum in the right halfplane. However, we affirm that the solution of this equation converges to zero in the uniform norm. This result seems counterintuitive, and it should be proved.

Proposition 3.1. Let $u_{0}(x)$ be a bounded integrable function. The solution of (3-1) on the whole axis with the initial condition $u(x, 0)=u_{0}(x)$ uniformly converges to 0 as $t \rightarrow \infty$.

Proof. Without loss of generality we can set $a=b=1$ and $c=0$. It follows from (3-2) that $|1-I(t)| \leq k_{1} e^{-t}$, where $k_{1}$ is a positive constant. Therefore, solution $u(x, t)$ of (3-1) can be estimated from above by the solution $u_{1}(x, t)$ of the equation

$$
\frac{\partial u}{\partial t}=D \frac{\partial^{2} u}{\partial x^{2}}+k_{1} e^{-t} u
$$

We will show that the solution of this equation with a bounded initial condition decaying at infinity uniformly converges to 0 . If the initial condition $u(x, 0)$ does not depend on $x$ and it equals some constant $k_{2}$, then its solution does not depend on $x$ either, and it satisfies the equation

$$
\frac{d v}{d t}=k_{1} e^{-t} v, \quad v(0)=k_{2} .
$$

We find $v(t)=k_{2} e^{k_{1}} e^{-k_{1} e^{-t}}$. Hence,

$$
|v(t)| \leq k_{2}, \quad t \geq 0,
$$

and a similar estimate holds for the solution $u_{1}(x, t)$ of (3-3). 
Consider a solution $u_{2}(x, t)$ of (3-3) with a bounded even positive integrable initial condition decaying at infinity. Then the solution is also an even positive function with a maximum at $x=0$. It is bounded by virtue of estimate (3-5).

We will prove that $u_{2}(0, t)$ converges to 0 as $t \rightarrow \infty$. Suppose that this is not the case. Then there exists $\epsilon>0$ such that

$$
u_{2}(0, t) \geq \epsilon
$$

for all $t$ sufficiently large. Indeed, if this is not true, then $u_{2}(0, t)$ converges to 0 along a sequence $t=t_{n}$. By virtue of the semigroup property of the solution and estimate (3-5), we conclude that $u_{2}(0, t)$ converges to 0 for all $t \rightarrow \infty$. This contradiction proves (3-6). Next, since the last term in the right-hand side of (3-3) converges to 0 as $t \rightarrow \infty$, then $u_{2}(x, t) \geq \epsilon / 2$ for $|x| \leq N(t)$, where $N(t) \rightarrow \infty$ as $t \rightarrow \infty$. Hence, $J(t)=\int_{-\infty}^{\infty} u_{2}(x, t) d x \rightarrow \infty$ as $t \rightarrow \infty$.

Integrating (3-3) with respect to $x$ from $-\infty$ to $\infty$, we obtain the equation for $J(t)$ :

$$
\frac{d J}{d t}=k_{1} e^{-t} J
$$

As above, we verify that its solution remains bounded. This contradiction proves the convergence $u_{2}(0, t) \rightarrow 0$. Since the maximum of this solution is reached at $x=0$, then $u_{2}(x, t)$ uniformly converges to 0 as $t \rightarrow \infty$.

It remains to note that any positive bounded integrable initial condition for (3-3) can be estimated from above by an even function satisfying the conditions above. Therefore, the solution with this initial condition uniformly converges to 0 .

It follows from this proposition that the solution of (3-1) with a constant immune response uniformly converges to 0 . If $c \geq a$, then $I(u)$ also vanishes for large time, while for $c<a$, it converges to a positive constant. This means that in the first case infection is completely eliminated, while in the second case, the total virus quantity remains constant. Furthermore, they do not form a localized solution in the space of genotypes corresponding to a virus strain but they diffuse in the genotype space covering a growing genotype range.

Increasing immune response. Clonal expansion of immune cells is stimulated by the antigen. Therefore, function $f(u)$ is increasing, at least for not very large values of $u$ for which excess of the virus can lead to the exhaustion of immune response. If $b=0$, then (3-1) is a conventional reaction-diffusion equation in the monostable case, and its solutions are described by reaction-diffusion waves.

Proposition 3.2. Suppose that $f(u)$ is a smooth growing function, $f(u)>0$ for $u>0$. Then (3-1) does not have a positive stationary solution with the zero limits at infinity. 
Proof. Suppose that (3-1) has a positive stationary solution $w(x)$ with the zero limits at infinity. Then it satisfies the problem

$$
w^{\prime \prime}+w(1-I(w)-f(w))=0, \quad w( \pm \infty)=0,
$$

where we set, without loss of generality, $D=a=b=1$. Then it has a maximum at some point $x=x_{m}, w_{m}=w\left(x_{m}\right)$. Let us verify that

$$
I(w)+f\left(w_{m}\right)<1 .
$$

Indeed, if $I(w)+f\left(w_{m}\right)>1$, then we obtain a contradiction in signs in (3-7) at $x=x_{m}$. If $I(w)+f\left(w_{m}\right)=1$, then by virtue of the uniqueness of solution, $w(x) \equiv w_{m}$, and the conditions at infinity cannot be satisfied.

Since $f(w)$ is an increasing function, it follows from (3-8) that the inequality $I(w)+f(w(x))<1$ holds for all $x \in \mathbb{R}$. Consider the equation $w^{\prime \prime}+w(k-f(w))=0$, where $k=1-I(w)>0$. Since $k-f(0)>0$, then the equation $w^{\prime \prime}+w(k-f(0))=0$, linearized about $w=0$, does not have positive solutions vanishing at infinity. This contradiction proves the proposition.

This proposition affirms that (3-1) does not have a positive stationary solution. Similar to Proposition 3.1, we can expect that the solution of the Cauchy problem uniformly converges to zero. This statement is not yet proved, and it represents an open question for future analysis.

Decreasing immune response. Since virus can kill immune cells and downregulate immune response (e.g., HIV), we consider here a decreasing function $f(u)$. In the case of a bounded interval $0<x<L$ and the integral $I(u)=\int_{0}^{L} u(x, t) d x,(3-1)$ has a constant stationary solution. It can lose its stability, resulting in the emergence of pulses (Figure 3). The bifurcation of pulses can be studied by the conventional stability and bifurcation analysis. This analysis is not applicable in the case of the whole axis.

Thus, the case of decreasing immune response is principally different in comparison with a constant or an increasing immune response. Depending on parameters, there can exist localized positive solutions corresponding to a virus strain.

We consider a model example where the existence of solutions can be proved.

Proposition 3.3. Let $f(u)=p-u$. Then there exist positive values $p_{1}, p_{2}, p_{1}<p_{2}$, such that (3-1) has a positive stationary solution decaying at infinity for $p_{1}<p<p_{2}$, and it does not have positive a solution for $0<p<p_{1}$ and $p>p_{2}$.

Proof. We look for a positive solution of problem (3-7). Set $k=1-I(w)$. Since $0<I(w)<1$, then $0<k<1$. Then problem (3-7) can be written as

$$
w^{\prime \prime}+w(k-f(w))=0, \quad w( \pm \infty)=0 .
$$



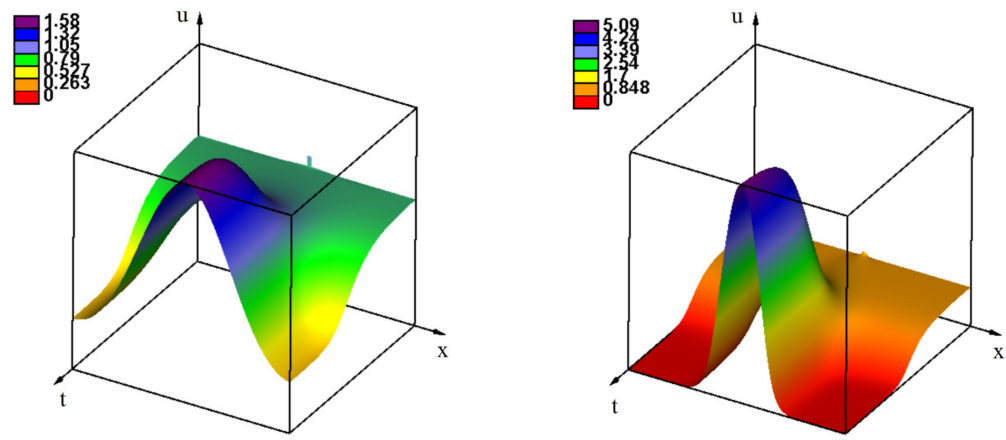

Figure 3. Convergence to the stationary solutions of (1-2) for $f(u)=k_{2} e^{-k_{3} u}$ and different values of the diffusion coefficient: $D=0.0015$ (left) and $D=0.0001$ (right). The values of other parameters are $L=1, a=b=1, k_{2}=0.2$, and $k_{3}=1$.

Existence of solution of this problem can be studied analytically. Suppose that such solution exists, and denote it by $w_{k}(x)$, where the subscript $k$ shows its dependence on the parameter $k$. Then we obtain the following equation with respect to $k$ :

$$
1-\int_{-\infty}^{\infty} w_{k}(x) d x=k
$$

Existence of its solution determines the existence of solution of problem (3-7). For $f(w)=b-w,(3-9)$ becomes

$$
w^{\prime \prime}-p w+w^{2}=0,
$$

where $p=b-k$. Set $w(x)=p v(\sqrt{p} x)$. Then $v(y)$ satisfies the equation

$$
v^{\prime \prime}-v+v^{2}=0 .
$$

It has a positive solution $v_{0}(y)$ such that $v_{0}( \pm \infty)=0$. Hence, $w_{k}(x)=p v_{0}(\sqrt{p} x)$, and from (3-10) we obtain

$$
I_{0} \sqrt{b-k}=1-k
$$

where $I_{0}=\int_{-\infty}^{\infty} v_{0}(y) d y$. Assertion of the proposition follows from the analysis of this equation.

The method of solutions presented here can be generalized for the functions $f(u)=b-u^{n}, n>1$.

3B. Interaction of genotype-dependent virus mortality and immune response. We can now study the interaction of immune response with the genotype-dependent virus mortality. If the function $\sigma(x)$ has two admissible intervals, then we showed 

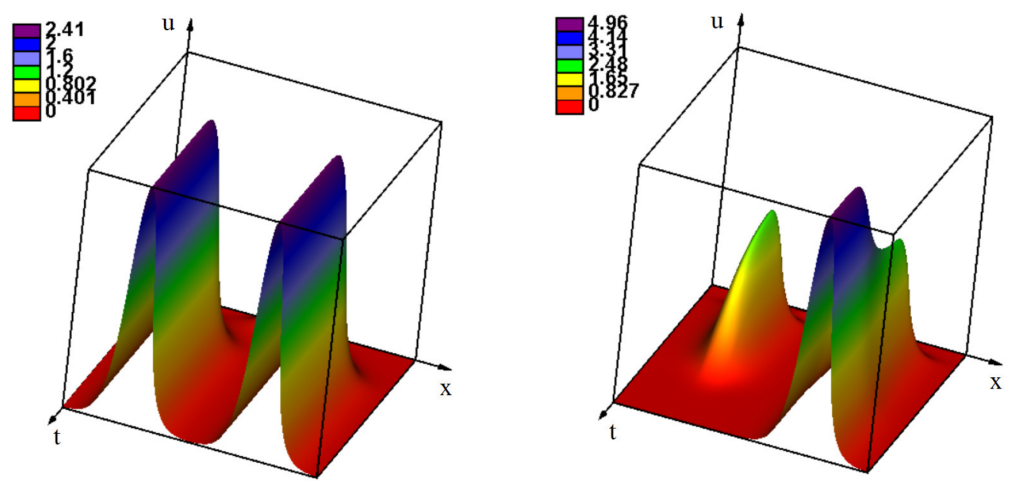

Figure 4. Numerical simulations of (1-2). In the case of symmetric initial condition, there is a bimodal virus density distribution with equal peaks (left). A small asymmetry in the initial condition leads to the disappearance of one peak and to the increase of the other one (right). The values of parameters are $L=1, a=b=1$, $D=0.001, \sigma(x)=0$ for $0.2<x<0.3$ and $0.7<x<0.8$ and $=1$ otherwise, initial condition $=0.1$ for $0.48<x<0.52$ (left) and $0.481<x<0.52$ (right), $f(u)=k_{2} e^{-k_{3} u}, k_{2}=0.2$, and $k_{3}=1$.

in Section 2B that two strains coexist. Their dynamics is described by intermediate solutions slowly convergent to the symmetric bimodal distribution. Immune response influences these dynamics, and this influence depends on the immune response function $f(u)$.

Increasing immune response. We begin the analysis of the influence of immune response on competing virus strains with the case of an increasing function $f(u)$. We consider for certainty a linear function, $f(u)=k_{1} u$. In this case, even if the initial condition is not symmetric, the solution rapidly converges to a symmetric distribution with equal peaks in the admissible intervals. The intermediate solutions observed before are not detected here.

Decreasing immune response. In the case of a decreasing function, $f(u)=k_{2} e^{-k_{3} u}$, the bimodal solution is symmetric in the case of a symmetric initial condition. However, a small asymmetry of the initial condition leads to the disappearance of one strain and to the increase of another one (Figure 4). Thus, the symmetric solution exists but is unstable.

Bell-shaped immune response. Consider the immune response function $f(u)=$ $k_{1} u e^{-k_{3} u}$ growing for small $u$ and decaying for large $u$. In the case of two admissible intervals, the solution can converge to a unimodal or to a bimodal distribution depending on the values of parameters (Figure 5). If $k_{3}$ is sufficiently small, then the growing branch of this function determines the behavior of solutions, and there 


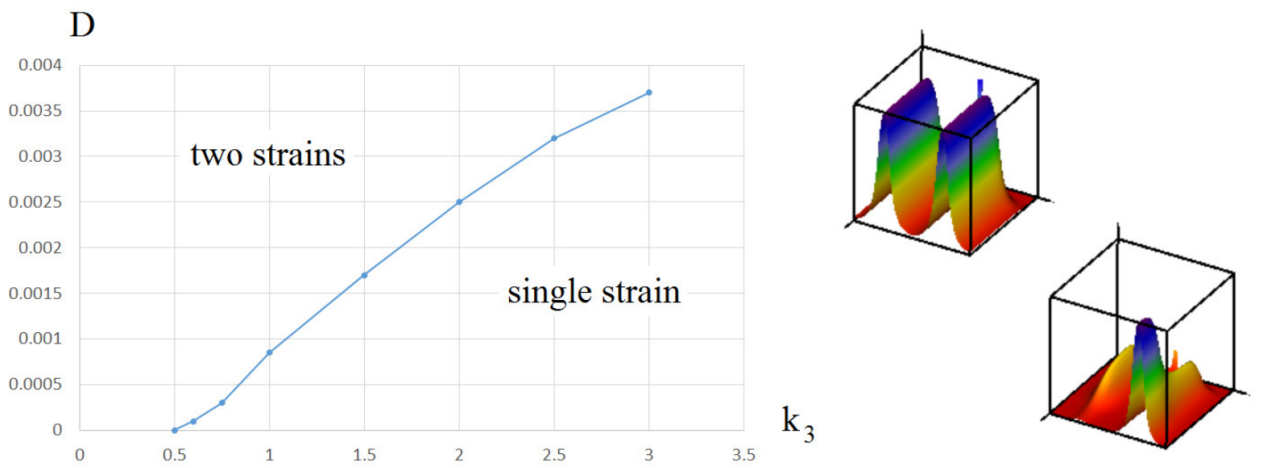

Figure 5. Numerical simulations of (1-2) in the case of bellshaped function $f(u)$ and two admissible intervals of the function $\sigma(x)$. Depending on the values of parameters, there are two persistent strains, or one of them vanishes. The values of parameters are $L=1, a=b=1, \sigma(x)=0$ for $0.2<x<0.3$ and $0.7<x<0.8$ and $=1$ otherwise, initial condition $=0.9$ for $0.481<x<0.52$, $f(u)=k_{1} u e^{-k_{3} u}$, and $k_{1}=1$. Two examples of the simulations at the right are carried out with $D=0.001, k_{3}=1$, and $k_{3}=1.2$.

are two persistent strains. If $k_{3}$ is large enough, then the decaying branch becomes dominating, and only one strain survives.

3C. The influence of treatment. In the case of two admissible intervals of the genotype-dependent mortality function $\sigma(x)$, there are two persistent strains rapidly converging to intermediate asymptotics depending on initial condition (Section 2B). Immune response can either preserve both strains or eliminate one (Section 3B).

We will now analyze how the competition of virus strains is influenced by a genotype-dependent virus treatment. We suppose that the function $\sigma$ depends on time,

$$
\sigma(x, t)= \begin{cases}\sigma_{0}(x), & 0 \leq t \leq t_{0} \\ \sigma_{1}(x), & t>t_{0} .\end{cases}
$$

Here $\sigma_{0}(x)$ is the original mortality rate, $t_{0}$ is the moment of time when treatment is applied, and $\sigma_{1}(x)$ is the mortality rate for which the effect of treatment is taken into account. In particular, treatment can eliminate one of the admissible intervals and influence the corresponding strain.

Let us illustrate the influence of treatment on the dynamics of virus strains by a simple case without immune response, $f(u) \equiv 0$. Consider two admissible intervals, $I_{1}=[0.2,0.3]$ and $I_{2}=[0.7,0.8]$, where $\sigma_{0}(x)=0$, and $\sigma_{0}(x)=1$ outside of these two intervals. The emergence of virus strains depends on the initial condition. If it is localized at the center of the interval $(0.49<x<0.51)$, then two equal strains 

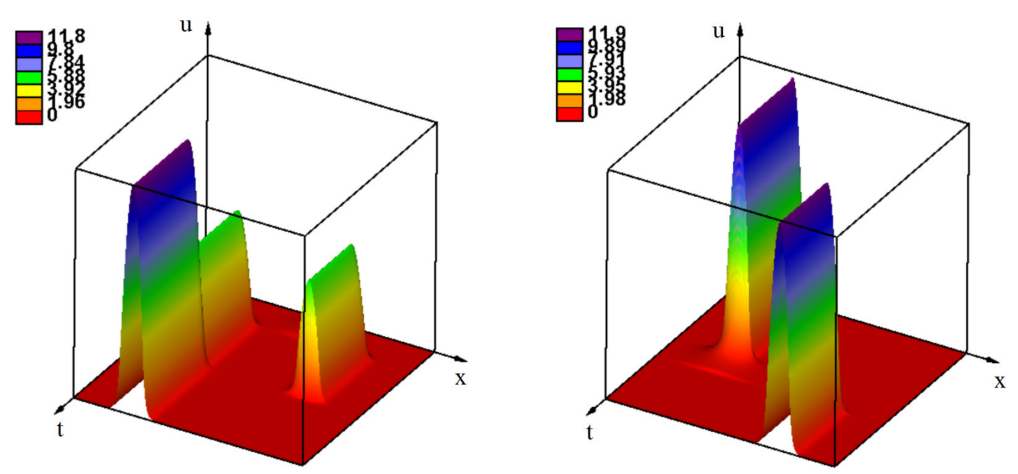

Figure 6. Numerical simulations of (1-2) without immune response and with a time-dependent mortality rate $\sigma$. In the case of two equal strains, treatment eliminates one of them and reinforces another one (left). In the case of a single strain, treatment eliminates it leading to the emergence of another strain (right). The values of parameters are $L=1, a=b=1, D=0.0001, \sigma(x)=0$ for $0.2<x<0.3$ and $0.7<x<0.8$ and $=1$ otherwise, initial condition $=0.1$ for $0.49<x<0.51$ (left) and $0.24<x<0.26$ (right), and $f(u) \equiv 0$.

emerge in the corresponding admissible intervals (Figure 6, left). At some moment of time $t=t_{0}$ we change the mortality rate to the function $\sigma_{1}(x)$ such that it equals 0 only in the first admissible interval, and it equals 1 in the second interval. Then the second strain rapidly disappears while the first strain grows. The total viral load (the integral of solution) does not change.

In the second case, the support of the initial condition is localized inside the first admissible interval, $0.24 \leq x \leq 0.26$. Only one strain emerges while another one is absent (cf. Section 2B). Applying treatment, we eliminate the first virus strain. After some time, the second strain appears (Figure 6, right). It could not appear before treatment because of the competition between the strains. Thus, an antiviral treatment can lead to the emergence of new strains. Moreover, the new strain is resistant to treatment since treatment acts on the first admissible interval but not on the second one.

\section{Discussion}

Virus mutation represents a big challenge for biomedical research and clinical medicine. There are hundreds of HIV mutants which can replace each other in the process of treatment. Resistant strains can emerge due to their natural evolution or due to antiviral treatment. On the other hand, virus evolution is an interesting 
object of theoretical studies. It has some features in common with the evolution of biological species, but it is faster and explicit in the sense that the virus itself is a relatively simple object, and its environment is also reduced to the host organism. Immune response of the host organism is very complex, but in the first approximation its consideration can be reduced to the multiplication of immune cells as a reaction to the antigen and to the elimination of the antigen.

Model. There are two main approaches to model virus mutations, discrete and continuous. In the discrete approach, there is a finite number of strains interacting with each other due to mutations (fluxes) and, possibly, due to the competition for host cells [Nowak and May 2000]. The corresponding ODE models resemble the models of competition of species in population dynamics. The advantage of such models is that they can be biologically realistic since virus strains and mutation characteristics can be taken from biological data. Furthermore, such models are relatively simple and easy to study in the case of two or three strains. However, they become very cumbersome for a large number of strains (equations), and their detailed analysis is literally impossible.

In continuous models, virus density distribution is considered as a function of genotype interpreted as a continuous variable [Kimura 1964; Sasaki 1994]. Though it is more difficult in this approach to describe a complex graph of virus strain connections by mutations, it is more appropriate to the investigation of the dynamics of this distribution. In this work we further develop this approach taking into account virus competition for host cells, immune response, and genotype-dependent mortality either natural or due to the antiviral treatment. This model is represented by the nonlocal reaction-diffusion equation (1-2), where the nonlocal term determines the virus multiplication rate. Indeed, the rate of cell infection is proportional to the virus density $u$ with the logistic limitation term $(1-b I(u))$ depending on the total virus quantity. It is similar to carrying capacity in population dynamics, and it determines the limitation on the total infection supported by the organism. Hence, the concentration of infected cells $C_{i}$ is described by the equation

$$
\frac{d C_{i}}{d t}=k u(1-b I(u))-\gamma C_{i},
$$

where the last term on the right-hand side characterizes death of infected cells. In the quasistationary approximation where the rates of cell infection and death are sufficiently high, we get $C_{i}=k / \gamma u(1-b I(u))$. Thus, the virus multiplication term, which is proportional to the concentration of infected cells $C_{i}$, can be written as $\operatorname{au}(1-I(u))$ (see (1-2)).

Let us note that there are two possible time delays in the model, one of them in the virus multiplication term and another one in the immune response term due to the clonal expansion of immune cells. Time delay in the immune response 
is taken into account in the reaction-diffusion models with time delay [Bessonov et al. 2020; Bocharov et al. 2016; Trofimchuk and Volpert 2018]. In this work we consider either stationary solutions of the corresponding equations or their longtime dynamics. Moreover, the characteristic diffusion time related to mutations is much longer than the characteristic time of virus multiplication or cell proliferation. In this case, the influence of time delay can be neglected.

Virus strains. From the modeling point of view, a virus strain can be represented by a density distribution concentrated around some genotype $x_{0}$ and rapidly decaying as the genotype $x$ goes away from $x_{0}$. A persistent virus strain corresponds to a positive stable stationary solution $u_{0}(x)$ with a maximum at some $x=x_{0}$. Existence of such solutions is not a priori known, and one of the objectives of our modeling study is to establish the conditions of the existence and stability of such solutions.

In our previous work [Bessonov et al. 2020], we showed that there are two mechanisms leading to the existence of stable stationary solutions of (1-2). One of them is determined by the admissible intervals where the virus mortality rate is low. Another one is related to the immune response. It is important to note here that the immune response function should have a decreasing branch. Otherwise, virus strains considered as positive stationary solutions of (1-2) decaying at infinity do not exist. For the existence of strains, admissible intervals should be sufficiently large and the virus mutation rate (diffusion coefficient) sufficiently small.

Competition of strains. The main goal of this work is to study the competition of virus strains emerging in two different admissible intervals. In the case without immune response, the behavior of strains should be considered in two time scales, fast and slow. In the fast time scale, they rapidly converge to some intermediate stationary solutions. The characteristic convergence time is on the order of 10 dimensionless time units. In the time scale of $10^{2}-10^{3}$ units, they do not practically change. The relative abundance of the two strains depends on the initial viral load (initial condition). Thus, there is a continuous family of intermediate stationary solutions determined by the initial condition.

In a slow time scale on the order of $10^{5}$ units, both strains become equal to each other. It should be noted that the characteristic fast and slow scales strongly depend on the diffusion coefficient. The values presented in our study are obtained for $D=10^{-3}$. The order of magnitude of the slow time scale rapidly grows with the decrease of the diffusion coefficient, and for $D=10^{-4}$ the limitation on the computational time does not allow us to reach it.

From the biomedical point of view, the existence of a family of intermediate solutions can bear important implications. In a short time scale, one needs to treat the strains determined by the initial viral load and not by their asymptotics for large time. 
We have shown that the mode of immune response strongly influences the behavior of solutions. A growing response function $f(u)$ eliminates the long scale dynamics, and the passage to the equal strains becomes fast. A decreasing response function eliminates one of the two competing strains. Finally, a bell-shaped function can have both effects depending on which of its two branches is dominating.

Limitations and perspectives. Equation (1-2) is derived under the assumption of consecutive mutations (see (1-3)). It can be considered as a small selection approximation of a more general model [Saakian et al. 2008] with a symmetric fitness function. The model considered in this work does not take into account complex intracellular regulation of virus reproduction and of immune response, the participation of different cells in the immune response, and some other aspects of virus-host interaction. On the other hand, this simplification allows us to reveal some general qualitative properties of virus evolution which might be impossible to predict in a more detailed model.

Overall, the presented modeling approach opens up interesting perspectives and allows various developments including time delay, other nonlocal terms, twodimensional problems, and so on.

\section{Appendix A: Stationary solution for two admissible intervals}

Consider the equation

$$
D u^{\prime \prime}+u(1-I(u))-\sigma(x) u=0
$$

on the whole axis, where $\sigma(x)=0$ for $x_{1} \leq|x| \leq x_{2}$ and $\sigma(x)=\sigma_{0}$ for $|x|<x_{1}$ and $|x|>x_{2}$. Here $\sigma_{0}, x_{1}$, and $x_{2}$ are some positive numbers, $x_{1}<x_{2}$. We will search for a nonzero bounded solution of this equation with zero limits at infinity.

Set

$$
1-I(u)=k^{2} .
$$

If $I(u) \geq 1$, then $u=0$ is the only bounded solution of (A-1). Hence, $0<I(u)<1$, and $k^{2}<1$. Then (A-1) can be written as

$$
\begin{array}{cl}
D u^{\prime \prime}+\left(k^{2}-\sigma_{0}\right) u & =0, \quad|x|<x_{1}, \quad|x|>x_{2}, \\
D u^{\prime \prime}+k^{2} u & =0, \quad x_{1} \leq|x| \leq x_{2} .
\end{array}
$$

Assuming that $k^{2}<\sigma_{0}$, we will look for its solution in the form

$$
u(x)=\left\{\begin{array}{lr}
c_{1} e^{\lambda x}, & x<-x_{2}, \\
c_{2} \cos (\mu x)+c_{3} \sin (\mu x), & -x_{2} \leq x \leq-x_{1}, \\
c_{4} e^{\lambda x}+c_{5} e^{-\lambda x}, & -x_{1}<x<x_{1}, \\
c_{6} \cos (\mu x)+c_{7} \sin (\mu x), & x_{1} \leq x \leq x_{2}, \\
c_{8} e^{-\lambda x}, & x>x_{2},
\end{array}\right.
$$


where

$$
\lambda=\sqrt{\sigma_{0}-k^{2}} / \sqrt{D}, \quad \mu=k / \sqrt{D} .
$$

From the continuity of the solution and of its first derivative,

$$
u\left( \pm x_{i}-0\right)=u\left( \pm x_{i}+0\right), \quad u^{\prime}\left( \pm x_{i}-0\right)=u^{\prime}\left( \pm x_{i}+0\right), \quad i=1,2,
$$

we get the equations

$$
\begin{aligned}
c_{1} e^{-\lambda x_{2}} & =c_{2} \cos \left(\mu x_{2}\right)-c_{3} \sin \left(\mu x_{2}\right), \\
c_{1} \lambda e^{-\lambda x_{2}} & =c_{2} \mu \sin \left(\mu x_{2}\right)+c_{3} \mu \cos \left(\mu x_{2}\right), \\
c_{4} e^{-\lambda x_{1}}+c_{5} e^{\lambda x_{1}} & =c_{2} \cos \left(\mu x_{1}\right)-c_{3} \sin \left(\mu x_{1}\right), \\
c_{4} \lambda e^{-\lambda x_{1}}-c_{5} \lambda e^{\lambda x_{1}} & =c_{2} \mu \sin \left(\mu x_{1}\right)+c_{3} \mu \cos \left(\mu x_{1}\right), \\
c_{4} e^{\lambda x_{1}}+c_{5} e^{-\lambda x_{1}} & =c_{6} \cos \left(\mu x_{1}\right)+c_{7} \sin \left(\mu x_{1}\right), \\
c_{4} \lambda e^{\lambda x_{1}}-c_{5} \lambda e^{-\lambda x_{1}} & =-c_{6} \mu \sin \left(\mu x_{1}\right)+c_{7} \mu \cos \left(\mu x_{1}\right), \\
c_{8} e^{-\lambda x_{2}} & =c_{6} \cos \left(\mu x_{2}\right)+c_{7} \sin \left(\mu x_{2}\right), \\
-c_{8} \lambda e^{-\lambda x_{2}} & =-c_{6} \mu \sin \left(\mu x_{2}\right)+c_{7} \mu \cos \left(\mu x_{2}\right) .
\end{aligned}
$$

Since we are looking for a nonzero solution, then the determinant of this system should be equal zero. This condition gives an equation with respect to $k$. The additional condition (A-2) will allow us to determine the coefficients $c_{i}$ and the solution. From (A-5) and (A-6), we get

$$
c_{2}=f_{2}(\lambda, \mu) c_{1}, \quad c_{3}=f_{3}(\lambda, \mu) c_{1},
$$

where

$$
\begin{aligned}
& f_{2}(\lambda, \mu)=e^{-\lambda x_{2}}\left(\mu \cos \left(\mu x_{2}\right)+\lambda \sin \left(\mu x_{2}\right)\right) / \mu, \\
& f_{3}(\lambda, \mu)=e^{-\lambda x_{2}}\left(-\mu \sin \left(\mu x_{2}\right)+\lambda \cos \left(\mu x_{2}\right)\right) / \mu .
\end{aligned}
$$

From (A-7) and (A-8),

$$
c_{4}=f_{4}(\lambda, \mu) c_{1}, \quad c_{5}=f_{5}(\lambda, \mu) c_{1},
$$

where

$$
\begin{aligned}
f_{4}(\lambda, \mu) & =\left(\left(\lambda \cos \left(\mu x_{1}\right)+\mu \sin \left(\mu x_{1}\right)\right) f_{2}+\left(-\lambda \sin \left(\mu x_{1}\right)+\mu \cos \left(\mu x_{1}\right)\right) f_{3}\right) e^{\lambda x_{1}} /(2 \lambda) \\
& =\left(2 \lambda \mu \cos \left(\mu\left(x_{2}-x_{1}\right)\right)+\left(\lambda^{2}-\mu^{2}\right) \sin \left(\mu\left(x_{2}-x_{1}\right)\right)\right) e^{\lambda\left(x_{1}-x_{2}\right)} /(2 \lambda \mu), \\
f_{5}(\lambda, \mu) & =\left(\left(\lambda \cos \left(\mu x_{1}\right)-\mu \sin \left(\mu x_{1}\right)\right) f_{2}-\left(\lambda \sin \left(\mu x_{1}\right)+\mu \cos \left(\mu x_{1}\right)\right) f_{3}\right) e^{-\lambda x_{1}} /(2 \lambda) \\
& =\left(\lambda^{2}+\mu^{2}\right) \sin \left(\mu\left(x_{2}-x_{1}\right)\right) e^{-\lambda\left(x_{1}+x_{2}\right)} /(2 \lambda \mu) .
\end{aligned}
$$

From (A-9) and (A-10), we get

$$
c_{6}=f_{6}(\lambda, \mu) c_{1}, \quad c_{7}=f_{7}(\lambda, \mu) c_{1},
$$


where

$$
\begin{aligned}
& f_{6}(\lambda, \mu)=\left(\left(f_{4} e^{\lambda x_{1}}+f_{5} e^{-\lambda x_{1}}\right) \mu \cos \left(\mu x_{1}\right)-\left(f_{4} e^{\lambda x_{1}}-f_{5} e^{-\lambda x_{1}}\right) \lambda \sin \left(\mu x_{1}\right)\right) / \mu \\
& \quad=f_{4}\left(\mu \cos \left(\mu x_{1}\right)-\lambda \sin \left(\mu x_{1}\right)\right) e^{\lambda x_{1}} / \mu+f_{5}\left(\mu \cos \left(\mu x_{1}\right)+\lambda \sin \left(\mu x_{1}\right)\right) e^{-\lambda x_{1}} / \mu, \\
& f_{7}(\lambda, \mu)=\left(\left(f_{4} e^{\lambda x_{1}}+f_{5} e^{-\lambda x_{1}}\right) \mu \sin \left(\mu x_{1}\right)+\left(f_{4} e^{\lambda x_{1}}-f_{5} e^{-\lambda x_{1}}\right) \lambda \cos \left(\mu x_{1}\right)\right) / \mu \\
& \quad=f_{4}\left(\mu \sin \left(\mu x_{1}\right)+\lambda \cos \left(\mu x_{1}\right)\right) e^{\lambda x_{1}} / \mu+f_{5}\left(\mu \sin \left(\mu x_{1}\right)-\lambda \cos \left(\mu x_{1}\right)\right) e^{-\lambda x_{1}} / \mu .
\end{aligned}
$$

From (A-11) and (A-12),

$$
c_{6} \lambda \cos \left(\mu x_{2}\right)+c_{7} \lambda \sin \left(\mu x_{2}\right)=c_{6} \mu \sin \left(\mu x_{2}\right)-c_{7} \mu \cos \left(\mu x_{2}\right) .
$$

Taking into account (A-15), we obtain

$$
\begin{aligned}
f_{4}\left(2 \lambda \mu \cos \left(\mu\left(x_{2}-x_{1}\right)\right)+\left(\lambda^{2}-\mu^{2}\right) \sin \left(\mu\left(x_{2}-x_{1}\right)\right)\right) e^{\lambda x_{1}} & \\
& =f_{5}\left(\lambda^{2}+\mu^{2}\right) \sin \left(\mu\left(x_{2}-x_{1}\right)\right) e^{-\lambda x_{1}} .
\end{aligned}
$$

Substituting the expressions for $f_{4}, f_{5}$, we obtain $f_{4}^{2}=f_{5}^{2}$, or

$2 \lambda \mu \cos \left(\mu\left(x_{2}-x_{1}\right)\right)+\left(\lambda^{2}-\mu^{2}\right) \sin \left(\mu\left(x_{2}-x_{1}\right)\right)= \pm\left(\lambda^{2}+\mu^{2}\right) \sin \left(\mu\left(x_{2}-x_{1}\right)\right) e^{-2 \lambda x_{1}}$.

Hence,

$$
\tan \left(\mu\left(x_{2}-x_{1}\right)\right)=\frac{2 \lambda \mu}{\mu^{2}-\lambda^{2} \pm\left(\mu^{2}+\lambda^{2}\right) e^{-2 \lambda x_{1}}} .
$$

This equality can be considered an equation with respect to $k$ :

$$
\tan \left(k\left(x_{2}-x_{1}\right) / \sqrt{D}\right)=\frac{2 k \sqrt{\sigma_{0}-k^{2}}}{2 k^{2}-\sigma_{0} \pm \sigma_{0} e^{-2 \sqrt{\sigma_{0}-k^{2}} x_{1} / \sqrt{D}}} .
$$

Let us note that the sign + in this equation corresponds to the symmetric solution and - to an asymmetric solution (see Figure 7). For $x_{1}=0$ we obtain the same equation as for the single admissible interval.

In order to describe the behavior of solutions of this equation under the variation of parameters, let us denote $h=\left(x_{2}-x_{1}\right) / \sqrt{D}$. We will consider $h$ as an independent parameter and will vary it for the other parameters $\sigma_{0}$ and $x_{1} / \sqrt{D}$ fixed. If $h$ is small enough, then (A-17) does not have solutions with $|k|<1$. If $h$ is greater than some critical value $h_{c}^{1}$, then there is a solution $|k|<1$ of the equation with sign + . In the interval $k_{c}^{1}<k<k_{c}^{2}$ for some other critical value $k_{c}^{2}$, there is only one solution. The second solution, corresponding to the equation with - appears for $k>k_{c}^{2}$ (Figure 8). The branch of solutions of the equation with - disappears for some $k_{c}^{3}$ because the right-hand side of this equation becomes infinite. On the other hand, another branch of tangent in the left-hand side of this equation provides a solution with a negative $k$. Next, the first branch of solutions for the equation with + disappears for some $k_{c}^{4}>k_{c}^{3}$, and it also reappears for $k<0$. 

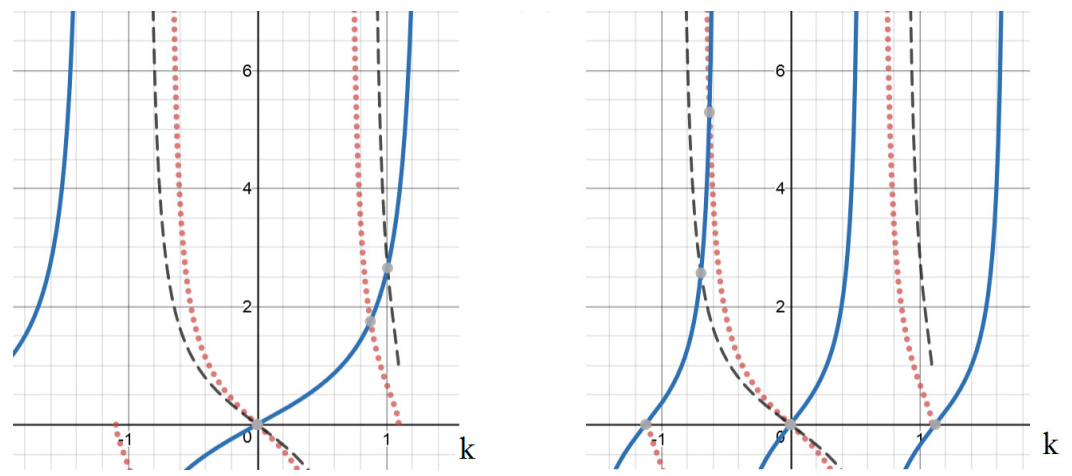

Figure 7. Graphical solution of (A-17) for the values of parameters $\sigma=1.2, x_{1} / \sqrt{D}=1,\left(x_{2}-x_{1}\right) / \sqrt{D}=1.2$ (left), and $=2.8$ (right). The function on the left-hand side of this equation is shown by a solid line, and the right-hand side with + is shown by a dotted line and with - by a dashed line.

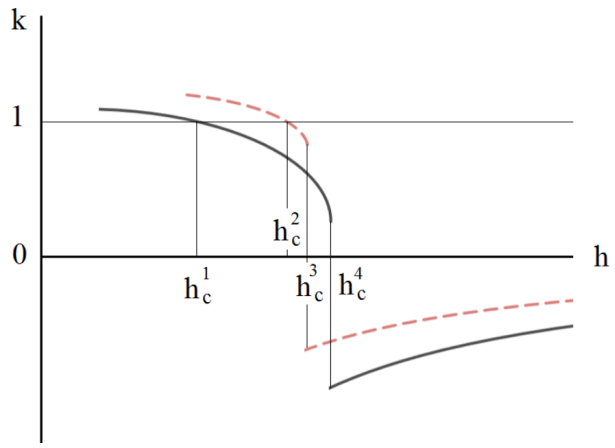

Figure 8. Schematic representation of the bifurcation diagram. The solid line corresponds to the solution of (A-17) with + and the dashed line to the solution of the equation with sign - .

Thus, this equation can have zero, one, or two solutions depending on $h$. Further increase of $h$ brings other branches of tangent and the number of solutions grows. However, we are interested only in positive and stable solutions. We expect that the branch of solutions of the equation with + is stable for small $h$, and with - for large $h$. There can exist an interval of bistability for $h_{c}^{3}<h<h_{c}^{4}$.

We can now find the integral $I(u)$. In the symmetric case,

$$
\begin{aligned}
I(u) & =2 \int_{-\infty}^{0} u(x) d x \\
& =2\left[\frac{c_{1}}{\lambda} e^{-\lambda x_{2}}+\frac{1}{\mu}\left(c_{2} \sin (\mu x)-c_{3} \cos (\mu x)\right)_{-x_{2}}^{x_{1}}+\frac{c_{4}}{\lambda}\left(e^{\lambda x}-e^{-\lambda x}\right)_{-x_{1}}^{0}\right] .
\end{aligned}
$$


$\mathrm{u}_{\max }$

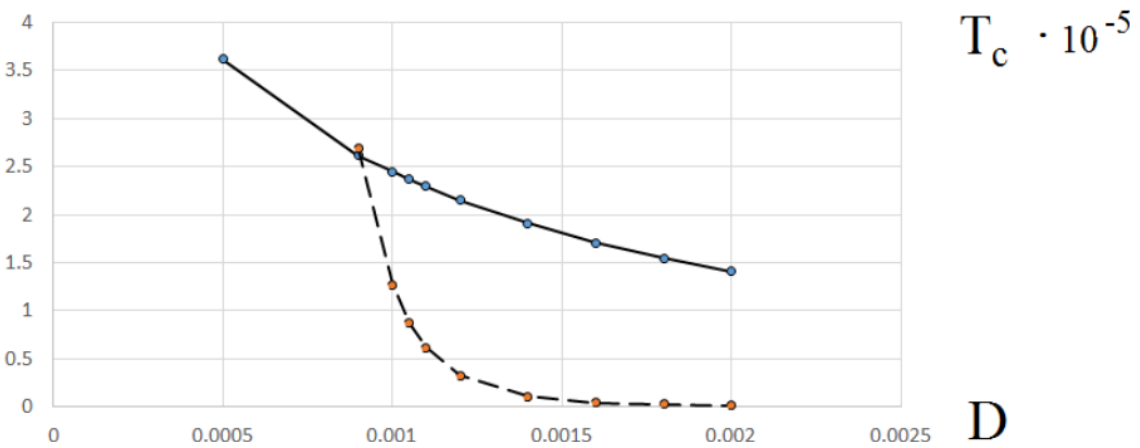

Figure 9. The maximal value of the stationary solution (solid line) and characteristic convergence time to the stationary solution (dashed line). The values of parameters are $L=1, a=b=1$, $D=0.001, \sigma(x)=0$ for $0.2<x<0.3$ and $0.7<x<0.8$ and $=1$ otherwise, and initial condition $=0.1$ for $0.49<x<0.52$.

From expressions (A-13), (A-14), and (A-15) and equality (A-2), we find $c_{1}$. Therefore, we can determine the coefficients $c_{2}, c_{3}, c_{4}$ and the solution $u(x)$. It can be done similarly in the nonsymmetric case.

Figure 9 shows the dependence of the stationary solution on the diffusion coefficient and of the convergence time to the stationary solution. We determine the convergence time $T_{c}$ as the time when the difference $\left|u_{m}^{1}-u_{m}^{2}\right|$ of the two maxima $u_{m}^{1}$ and $u_{m}^{2}$ of the pulses becomes less than 0.01 . The convergence time rapidly increases as the diffusion coefficient decreases, and the simulation time becomes too large for $D<0.001$.

\section{Appendix B: Numerical implementation}

In numerical simulations we consider the equation

$$
\frac{\partial u}{\partial t}=D \frac{\partial^{2} u}{\partial x^{2}}+a u(1-b I(u))-u f(u)-\sigma(x) u
$$

on a bounded interval $0<x<L$ with two different realizations:

- Periodic boundary conditions, and the integral is given by the formula

$$
I(u)=\frac{1}{2 N} \int_{x-N}^{x+N} u(x, t) d x .
$$

If the limits of the integral go beyond the interval $[0, L]$, then the function $u(x, t)$ is continued by periodicity. 
- Neumann boundary conditions, and the integral is given by the formula

$$
I(u)=\int_{0}^{L} u(x, t) d x .
$$

The numerical results presented in the work have been obtained with the first method. We have verified that the second method gives similar results.

\section{Appendix C: Proof of Theorem 2.2}

Consider the equation

$$
u^{\prime \prime}+u(1-I(u))-\sigma(x) u=0
$$

on the whole axis, where $I(u)=\int_{-\infty}^{\infty} u(x) d x$ and $\sigma(x)$ is a bounded nonnegative sufficiently smooth function. We look for positive solutions of this equations with zero limits at infinity. We will apply here the topological degree method. We begin with a priori estimates of solutions.

Lemma C.1. Let $u(x)$ be a positive solution of $(\mathrm{C}-1), u( \pm \infty)=0$. Then $I(u)<1$.

The proof of the lemma follows directly from the maximum principle. Indeed, if $I(u) \geq 1$, then $u(x)$ is a solution of the equation $u^{\prime \prime}+q(x) u=0$ with $q(x) \leq 0$ and $q(x) \not \equiv 0$. Therefore, $u(x)$ cannot have positive maximum or negative minimum. Hence, $u(x) \equiv 0$.

Lemma C.2. Suppose that $\sigma(x)=\sigma_{0}>1$ for $|x| \geq x_{1}$ with some positive $\sigma_{0}$ and $x_{1}$. Then $u\left(x_{1}\right)<\sqrt{\sigma}_{0} / 2$.

Proof. For $x \geq x_{1}$, (C-1) gives $u^{\prime \prime}-a u=0$, where $a=\sigma_{0}-(1-I(u))<\sigma_{0}, a>0$. Then

Hence,

$$
u(x)=u\left(x_{1}\right) e^{-\sqrt{a}\left(x-x_{1}\right)}, \quad \int_{x_{1}}^{\infty} u(x) d x=\frac{u\left(x_{1}\right)}{\sqrt{a}}>\frac{u\left(x_{1}\right)}{\sqrt{\sigma_{0}}} .
$$

$$
1>I(u)>2 \int_{x_{1}}^{\infty} u(x) d x>\frac{2 u\left(x_{1}\right)}{\sqrt{\sigma_{0}}} .
$$

This inequality proves the lemma.

Lemma C.3. Suppose that $\sigma(x)$ is a continuous function and $\sup _{x} \sigma(x) \leq M$. Then a positive solution $u(x)$ admits an estimate which depends only on $M$.

Proof. The solution $u(x)$ of (C-1) satisfies the boundary problem

$$
v^{\prime \prime}+b(x) v=0, \quad v\left( \pm x_{1}\right)=u\left( \pm x_{1}\right),
$$

on the interval $-x_{1} \leq x \leq x_{1}$. Here $b(x)=1-I(u)-\sigma(x)$ is a bounded continuous function, $|b(x)| \leq M+1 \equiv m$. According to the previous lemma, the boundary 
values of the solution are bounded. Therefore, it is sufficient to estimate a maximum of the solution inside the interval. Suppose that the function $v(x)$ has a global maximum at some point $x_{0} \in\left[-x_{1}, x_{1}\right]$. Then

$$
\left|v^{\prime}(x)\right|=\left|\int_{x_{0}}^{x} v^{\prime \prime}(y) d y\right| \leq m v\left(x_{0}\right)\left|x-x_{0}\right| .
$$

Hence,

$$
v(x)=v\left(x_{0}\right)+\int_{x_{0}}^{x} v^{\prime}(y) d x \geq v\left(x_{0}\right)-\frac{1}{2} m v\left(x_{0}\right)\left(x-x_{0}\right)^{2}=v\left(x_{0}\right) g(x),
$$

where $g(x)=\frac{1}{2}-m\left(x-x_{0}\right)^{2}$. Denote by $\Omega$ the interval in $\left[-x_{1}, x_{1}\right]$ where this function is positive. Then $\int_{\Omega} g(x) d x \geq \kappa>0$, where the constant depends only on $M$ and possibly on $x_{1}$. Hence, $1>I(v)>\kappa v\left(x_{0}\right)$. This estimate proves the lemma.

We will use the topological degree theory to prove the existence of solutions [Volpert 2014]. Lemma C.3 above provides a priori estimates of solutions. Consider the operator

$$
A_{\theta}(u)=u^{\prime \prime}+u(1-I(u))-\sigma_{\theta}(x) u,
$$

acting from the Hölder space $C^{2+\alpha}(\mathbb{R})$ into the space $C^{\alpha}(\mathbb{R})$. Here $0<\alpha<1$ and $\theta \in[0,1]$ is a parameter. We will suppose for simplicity that $\sigma_{\theta}(x)$ is an infinitely differentiable function with respect to $x$ and $\theta$. Other conditions will be specified later.

Denote by $L_{\theta}$ the operator obtained by linearization of the operator $A_{\theta}(u)$ about $u=0$ :

$$
L_{\theta} v=v^{\prime \prime}+v-\sigma_{\theta}(x) v .
$$

Lemma C.4. Suppose that the principal eigenvalue of the operator $L_{\theta}$ is positive for $\theta_{0} \leq \theta \leq \theta_{1}$ and for some $\theta_{0}, \theta_{1}$. Then there exists $\epsilon>0$ such that $u_{m}=$ $\sup _{x} u(x) \geq \epsilon$ for any positive solution of the equation $A_{\theta}(u)=0, \theta_{0} \leq \theta \leq \theta_{1}$.

Proof. Suppose that the assertion of the lemma does not hold and there is a sequence of solutions $u_{k}(x)$ for $\theta=\theta_{k}$ such that $u_{m_{k}} \rightarrow 0$. Without loss of generality we can assume that $\theta_{k} \rightarrow \theta_{*}$ for some $\theta_{*} \in\left[\theta_{0}, \theta_{1}\right]$. Then

$$
0=A_{\theta_{k}}\left(u_{k}\right)=A_{\theta_{k}}(0)+L_{\theta_{k}} u_{k}+o\left(\left\|u_{k}\right\|\right)=L_{\theta_{k}} u_{k}+o\left(\left\|u_{k}\right\|\right) .
$$

Set $v_{k}=u_{k} /\left\|u_{k}\right\|$. Then $L_{\theta_{k}} v_{k}=o(1)$. Since $L_{\theta_{k}}$ is proper with respect to $v$ and $\theta$, the sequence $v_{k}$ is compact and we can choose a convergent subsequence $v_{k} \rightarrow v_{0}$. Hence, $L_{\theta_{*}} v_{0}=0$. Since the functions $u_{k}(x)$ are positive, then $v_{0}(x)>0$ for all $x$. Therefore, the operator $L_{\theta_{*}}$ has a zero eigenvalue with a positive eigenfunction. However, the only positive eigenfunction corresponds to the principal eigenvalue. We obtain a contradiction with the assumption that the principal eigenvalue of the operator $L_{\theta^{*}}$ is positive. 
Theorem C.5. Suppose $\sigma(x)=\sigma_{0}>1$ for $|x| \geq x_{1}$ with some positive $\sigma_{0}$ and $x_{1}$, and the principal eigenvalue of the problem

$$
u^{\prime \prime}+u-\sigma(x) u=\lambda u
$$

is positive. Then (C-1) has a positive solution converging to 0 at infinity.

Proof. Set $\sigma_{\theta}(x)=(1-\theta) \sigma(x)+\theta \sigma_{0}$. Since $\sigma_{0}>1$, then the operator $L_{1}$ has the spectrum in the left half-plane. Let us note that the essential spectrum $S_{e}\left(L_{\theta}\right)$ of the operator $L_{\theta}$ does not depend on $\theta$, and $\operatorname{Re} S_{e}\left(L_{\theta}\right) \leq-\delta<0$ for some positive $\delta$. Denote the principal eigenvalue of this operator, that is, the eigenvalue with the maximal real part, by $\lambda_{0}(\theta)$. According to the assumption of the theorem $\lambda_{0}(0)>0$. It is a monotonically decreasing function of $\theta \in[0,1]$, and there exists such $\theta_{0} \in[0,1]$ that

$$
\lambda_{0}\left(\theta_{0}\right)=0, \quad \lambda_{0}(\theta)>0 \text { for } 0<\theta \leq \theta_{0}, \quad \lambda_{0}(\theta)<0 \text { for } \theta_{0}<\theta \leq \theta_{1} .
$$

Here $\theta_{1}$ is some value in the interval $\left(\theta_{0}, 1\right]$. Since the eigenvalue can approach the essential spectrum, we cannot guarantee its existence for all $\theta \in[0,1]$.

Let us consider the equation $A_{\theta}(u)=0$ in a small vicinity of the bifurcation point $\theta=\theta_{0}$. For this value of parameter, the trivial solution $u=0$ loses its stability, leading to the appearance of another solution $u_{\theta}(x)$. This solution is positive since the principal eigenfunction $v_{0}(x)$ is positive [Volpert and Volpert 2000]. Furthermore, the index of this solution, that is, the value of the degree with respect to a small ball containing this solution, equals 1 . Indeed, from the homotopy invariance of the degree, it follows that

$$
\operatorname{ind}(0)+\operatorname{ind}\left(u_{\theta}\right)+\operatorname{ind}\left(\tilde{u}_{\theta}\right)=1
$$

for all $\theta>\theta_{0}$ and sufficiently close to $\theta_{0}$. Here $\tilde{u}_{\theta}(x)$ is a negative solution bifurcating from the trivial solution and approaching $-v_{0}(x)$. Since ind $(0)=-1$ because it equals $(-1)^{v}$, where $v=1$ is the number of positive eigenvalues of the linearized operator, then $\operatorname{ind}\left(u_{\theta}\right)=\operatorname{ind}\left(\tilde{u}_{\theta}\right)=1$.

It follows from Lemma C.3 that $\|u\|_{C^{2+\alpha}(\mathbb{R})}<M_{0}$ for some positive constant $M_{0}$ and for any positive solution $u$ of the equation $A_{\theta}(u)=0$. Next, from Lemma C. 4 we conclude that $\|u\|_{C^{2+\alpha}(\mathbb{R})}>\delta(\theta)$ for some positive $\delta(\theta), \theta<\theta_{0}$. Consider the domain

$$
\Omega=\left\{u \in C^{2+\alpha}(\mathbb{R}) \mid u(x)>0, x \in \mathbb{R}, \delta_{0}<\|u\|_{C^{2+\alpha}(\mathbb{R})}<M_{0}\right\}
$$

for some $\delta_{0}>0$ sufficiently small. Choose $\theta_{2}<\theta_{0}$ such that $\delta(\theta)>\delta_{0}$ for $0 \leq \theta \leq \theta_{2}$. Since $A_{\theta}(u) \neq 0$ for $u \in \partial \Omega, 0 \leq \theta \leq \theta_{2}$, then the value of the degree $\gamma\left(A_{\theta}, \Omega\right)$ does not depend on $\theta \in\left[0, \theta_{2}\right]$. Hence, $\gamma\left(A_{0}, \Omega\right)=\gamma\left(A_{\theta_{2}}, \Omega\right)=\operatorname{ind}\left(u_{\theta_{2}}\right)=1$, and equation $A_{0}(u)=0$ has a solution in $\Omega$. 


\section{Acknowledgements}

This research was funded by the Russian Science Foundation (grant number 1811-00171) for N. Bessonov and G. Bocharov. V. Popov and V. Volpert were partly supported by the Peoples' Friendship University of Russia Program 5-100.

\section{References}

[Bessonov et al. 2020] N. Bessonov, G. Bocharov, A. Meyerhans, V. Popov, and V. Volpert, "Nonlocal reaction-diffusion model of viral evolution: emergence of virus strains", Mathematics 8:1 (2020), art. id. 117.

[Biebricher and Eigen 2006] C. K. Biebricher and M. Eigen, "What is a quasispecies?", Curr. Top. Microbiol. Immunol. 299 (2006), 1-31.

[Bocharov et al. 2005] G. Bocharov, N. J. Ford, J. Edwards, T. Breinig, S. Wain-Hobson, and A. Meyerhans, "A genetic-algorithm approach to simulating human immunodeficiency virus evolution reveals the strong impact of multiply infected cells and recombination", J. Gen. Virol. 86:11 (2005), 3109-3118.

[Bocharov et al. 2016] G. Bocharov, A. Meyerhans, N. Bessonov, S. Trofimchuk, and V. Volpert, "Spatiotemporal dynamics of virus infection spreading in tissues", PLoS One 11:12 (2016), art. id. e0168576.

[Bocharov et al. 2018] G. Bocharov, V. Volpert, B. Ludewig, and A. Meyerhans, Mathematical immunology of virus infections, Springer, 2018.

[Coffin and Swanstrom 2013] J. Coffin and R. Swanstrom, "HIV pathogenesis: dynamics and genetics of viral populations and infected cells", Cold Spring Harb. Perspect. Med. 3:1 (2013), art. id. a012526.

[Domingo and Perales 2018] E. Domingo and C. Perales, "Quasispecies and virus", Eur. Biophys. J. 47:4 (2018), 443-457.

[Eigen 1971] M. Eigen, "Selforganization of matter and the evolution of biological macromolecules", Naturwissenschaften 58:10 (1971), 465-523.

[Ganusov et al. 2011] V. V. Ganusov, N. Goonetilleke, M. K. Liu, G. Ferrari, G. M. Shaw, A. J. McMichael, P. Borrow, B. T. Korber, and A. S. Perelson, "Fitness costs and diversity of the cytotoxic T lymphocyte (CTL) response determine the rate of CTL escape during acute and chronic phases of HIV infection", J. Virol. 85:20 (2011), 10518-10528.

[Gaudieri et al. 2009] S. Gaudieri, A. Rauch, K. Pfafferott, E. Barnes, W. Cheng, G. McCaughan, N. Shackel, G. P. Jeffrey, L. Mollison, R. Baker, H. Furrer, H. F. Günthard, E. Freitas, I. Humphreys, P. Klenerman, S. Mallal, I. James, S. Roberts, D. Nolan, and M. Lucas, "Hepatitis C virus drug resistance and immune-driven adaptations: relevance to new antiviral therapy", Hepatology 49:4 (2009), 1069-1082.

[Kimura 1964] M. Kimura, "Diffusion models in population genetics", J. Appl. Probability 1 (1964), 177-232.

[Martínez et al. 2011] J. P. Martínez, G. Bocharov, A. Ignatovich, J. Reiter, M. T. Dittmar, S. WainHobson, and A. Meyerhans, "Fitness ranking of individual mutants drives patterns of epistatic interactions in HIV-1", PLoS One 6:3 (2011), art. id. e18375.

[McMichael and Carrington 2019] A. J. McMichael and M. Carrington, "Topological perspective on HIV escape", Science 364:6439 (2019), 438-439. 
[Nowak and May 2000] M. A. Nowak and R. M. May, Virus dynamics: mathematical principles of immunology and virology, Oxford University, 2000.

[Rouzine et al. 2001] I. M. Rouzine, A. Rodrigo, and J. M. Coffin, "Transition between stochastic evolution and deterministic evolution in the presence of selection: general theory and application to virology”, Microbiol. Mol. Biol. Rev. 65:1 (2001), 151-185.

[Saakian et al. 2008] D. B. Saakian, O. Rozanova, and A. Akmetzhanov, "Dynamics of the eigen and the Crow-Kimura models for molecular evolution", Phys. Rev. E (3) 78:4 (2008), art. id. 041908.

[Sasaki 1994] A. Sasaki, "Evolution of antigen drift/switching: continuously evading pathogens", $J$. Theor. Biol. 168:3 (1994), 291-308.

[Trofimchuk and Volpert 2018] S. Trofimchuk and V. Volpert, "Traveling waves for a bistable reactiondiffusion equation with delay”, SIAM J. Math. Anal. 50:1 (2018), 1175-1199.

[Vijay et al. 2008] N. N. V. Vijay, Vasantika, R. Ajmani, A. S. Perelson, and N. M. Dixit, "Recombination increases human immunodeficiency virus fitness, but not necessarily diversity", J. Gen. Virol. 89:6 (2008), 1467-1477.

[Volpert 2014] V. Volpert, Elliptic partial differential equations, vol. 2: Reaction-diffusion equations, Monographs in Mathematics 104, Springer, 2014.

[Volpert and Volpert 2000] A. I. Volpert and V. A. Volpert, "Spectrum of elliptic operators and stability of travelling waves", Asymptot. Anal. 23:2 (2000), 111-134.

Received 13 Sep 2019. Revised 9 Jan 2020. Accepted 18 Feb 2020.

NIKOLAI BESSONOV: nickbessonov1@gmail.com

Institute of Problems of Mechanical Engineering, Russian Academy of Sciences, Saint Petersburg, Russia

and

Marchuk Institute of Numerical Mathematics, Russian Academy of Sciences, Moscow, Russia

GENNADY A. BOCHAROV: g. bocharov@inm.ras.ru

Marchuk Institute of Numerical Mathematics, Russian Academy of Sciences, Moscow, Russia and

Gamaleya Center of Epidemiology and Microbiology, Moscow, Russia

CRISTINA LEON: merycris25@hotmail.com

Peoples' Friendship University of Russia, Moscow, Russia

VLADIMIR POPOV: volodimir.a@gmail.com

Peoples' Friendship University of Russia, Moscow, Russia

VITALY VOLPERT: volpert@math.univ-lyon1.fr

Institut Camille Jordan, UMR 5208 CNRS, University Lyon 1, Villeurbanne, France

and

Team Dracula, Institut National de Recherche en Informatique et en Automatique, Lyon La Doua, Villeurbanne, France

and

Marchuk Institute of Numerical Mathematics, Russian Academy of Sciences, Moscow, Russia and

Peoples' Friendship University of Russia, Moscow, Russia 


\title{
MODELING THE LINEAR DYNAMICS OF CONTINUOUS VISCOELASTIC SYSTEMS ON THEIR INFINITE-DIMENSIONAL CENTRAL SUBSPACE
}

\author{
Angelo Luongo And Francesco D’Annibale
}

\begin{abstract}
A metamodel of linear viscoelastic continuum is formulated. Internal variables, of arbitrary number, are introduced to describe the viscous part of the strain, and a wide class of constitutive laws, suggested by rheological models, is considered. The spectral properties of the system are discussed. Based on the separation of the eigenvalues occurring when the viscous moduli are small, the system is reduced to its infinite-dimensional central subspace, on which the steady dynamics takes place. Both the center manifold method and the multiple scales method are used to build the reduced model, which is formulated in terms of the only observable variables. Examples relevant to one-, two-, and three-dimensional continua are worked out to illustrate the theory, in conjunction with the standard three-parameter model and the five-parameter model.
\end{abstract}

\section{Introduction}

Linear viscoelasticity, both in statics and dynamics, has often been considered a "simple" matter, since it's somewhat "similar" to linear elasticity. In fact, a well known elastic-viscoelastic correspondence principle [Flügge 1975] states that the two problems are formally equal when they are both transformed in the Laplace or Fourier domains. Thus, transformation is believed to be the best way to study viscoelastic problems, although often nontrivial antitransformations are needed when one desires to build the response time history [Narayanan and Beskos 1982].

There exist, of course, studies in which the system dynamics is analyzed in the time domain, for discrete or discretized systems, via finite or boundary elements methods (see, e.g., [Golla and Hughes 1985; Sim and Kwak 1988; McTavish and Hughes 1993; Schanz 1999; 2001; Hatada et al. 2000; Lewandowski et al. 2012; Syngellakis 2003; Baroudi et al. 2019]). An example of analysis which makes use of an the internal variable formulation is given in [Lewandowski et al. 2012]. All

\section{Communicated by Francesco dell'Isola.}

MSC2010: 74D05, 74H10, 74H40, 74H45, 74Q10.

Keywords: continuous viscoelastic metamodel, internal variables, linear dynamics, center manifold, multiple scales method, viscoelastic beam on viscoelastic Winkler soil, viscoelastic plate, viscoelastic Cauchy continuum. 
these studies, however, seem to not completely exploit the spectral characteristics of viscoelastic systems when damping, as usual, is small. In this case, indeed, the dynamics is made of a fast transient phase, and a successive steady-state phase, which takes place in a space of reduced dimensions, equal to those of the elastic system. This property was instead used by the authors in a recent paper [Luongo and D'Annibale 2017a], where a reduction method was proposed to contract the dimension of the discrete system to that of the associated elastic system. The procedure was based on the center manifold method, which is a common tool used in bifurcation analysis, designed to tackle nonlinear systems, and adapted in [Luongo and D'Annibale 2017a] to linear systems (similarly to what was done in [Shaw and Pierre 1991] in dealing with nonlinear normal modes).

The method discussed in [Luongo and D'Annibale 2017a] sheds light on another form of the correspondence principle which does not seem to have been explored yet, i.e., on a similarity holding not only in the transformed domains (where it exactly holds), but also in the time domain, although in the context of an asymptotic (and, therefore, approximate) theory. However, the procedure of [Luongo and D'Annibale 2017a] was limited to discrete systems, and did not seem, at first glance, to be straightforwardly extendible to infinite-dimensional systems. As a matter of fact, the center manifold theorem has only been proved for finitedimensional central subspaces (i.e., for a finite number of eigenvalues lying on the imaginary axis, or close to it). However, a favorable circumstance exists in the viscoelastic case, namely that the whole space of displacement and velocities is of interest. In other words, we do not have to worry about describing an infinitedimensional subspace in which a subset of displacements and velocities appear (as, for example, would be the case for a system undergoing an infinite number of buckling modes), but we have to take all the displacements and velocities which are admissible for the model. In this paper, we will prove (by a heuristic approach) that this circumstance still allows the use of the center manifold theorem.

The paper is organized as follows. In Section 2 a continuous viscoelastic metamodel using internal variables is formulated. In Section 3 both the center manifold and the multiple scales methods are applied to build a reduced system. In Sections 4, 5, and 6 sample systems, of increasing complexity, are worked out, and their reduced counterparts are derived. In Section 7 some conclusions are presented. Finally, in the Appendix an illustrative numerical example, concerning a homogenized microstructured viscoelastic one-dimensional continuum, is detailed.

\section{Continuous viscoelastic metamodel}

A dynamic linear metamodel of viscoelastic continuum is formulated. To this end a matrix notation is adopted, by denoting algebraic matrices by bold roman 

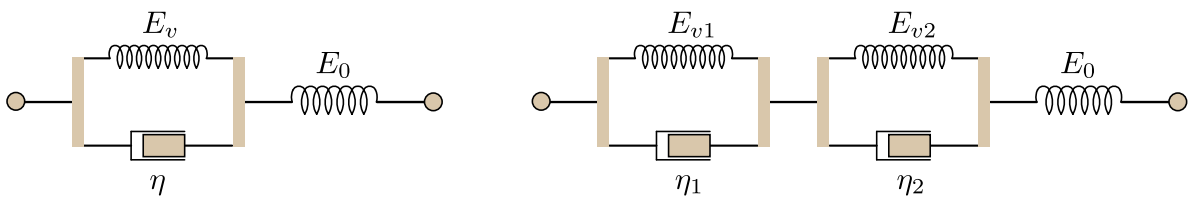

Figure 1. Rheological solid models: standard three-parameter (left) and five-parameter (right).

characters and formal matrices, made of linear differential operators, by bold calligraphic characters.

Kinematics and equilibrium of the system are governed by

$$
\begin{aligned}
\mathcal{D} u & =\boldsymbol{e}, \\
\mathcal{D}^{a} s & =f(x, t)-M \ddot{u},
\end{aligned}
$$

which are constrained by geometrical and mechanical boundary conditions. Here, $\boldsymbol{u}(\boldsymbol{x}, t), \boldsymbol{e}(\boldsymbol{x}, t), \boldsymbol{s}(\boldsymbol{x}, t)$ are column matrices collecting displacements, strains, and stress fields, respectively, and depending on position $\boldsymbol{x}$ and time $t ; \mathcal{D}$, and its adjoint $\mathcal{D}^{a}$, are the kinematic and equilibrium operators, collecting derivatives with respect to the coordinates $\boldsymbol{x} ; \boldsymbol{f}(\boldsymbol{x}, t)$ is the column matrix of the active forces; $\boldsymbol{M}$ is the inertia matrix; and the dot denotes differentiation with respect to the time $t$.

A class of viscoelastic constitutive laws is considered, which can be derived through kinematics and equilibrium and constitutive equations of rheological models. These latter are multiparameter solid models, made of Kelvin-Voigt (in-parallel spring/dashpot) elements, which are assembled in series to a spring device. Accordingly, the constitutive law reads

$$
\begin{aligned}
\boldsymbol{s} & =\boldsymbol{C}_{e}\left(\boldsymbol{e}-\boldsymbol{e}_{v}\right), \\
\boldsymbol{e}_{v} & =\boldsymbol{\Omega} \boldsymbol{\kappa}, \\
\dot{\boldsymbol{\kappa}} & =\boldsymbol{\Lambda}_{e} \boldsymbol{e}-\boldsymbol{\Lambda}_{R} \boldsymbol{\kappa} .
\end{aligned}
$$

Here, $\boldsymbol{e}_{v}$ is the viscous part of the strain, so that $\boldsymbol{e}-\boldsymbol{e}_{v}$ is the elastic part; stresses are assumed to be proportional to these latter parts by way of the squared elastic matrix $\boldsymbol{C}_{e}=\boldsymbol{C}_{e}^{T}$. The viscous strains, however, are linearly dependent on a generally larger number of internal variables $\boldsymbol{\kappa}$ [Moreau 1970; Maugin and Muschik 1990; Lemaitre and Chaboche 1990], to which they are connected via the rectangular matrix $\boldsymbol{\Omega}$, accounting for the topology of the underlying rheological model. For example, if reference is made to the standard three-parameter model (Figure 1, left), then $e_{v}$ and $\kappa$ coincide, being equal to the elongation $\varepsilon_{\mathrm{KV}}$ of the KelvinVoigt submodel; if, instead, the five-parameter model is adopted (Figure 1, right), $e_{v}=\kappa_{1}+\kappa_{2}$, with $\kappa_{i}(i=1,2)$ the elongation of the $i$-th Kelvin-Voigt submodel. 
Equation $(2)_{1}$ is the state law, while $(2)_{3}$ is the flow law, governing the evolution of the internal variables, here taken as linear; in it, $\boldsymbol{\Lambda}_{e}, \boldsymbol{\Lambda}_{R}$ are matrices of material constants, having dimension of the inverse of time, here referred to as relaxation matrices.

By combining (1) and (2) the following equations of motion, in state form, are derived:

$$
\left(\begin{array}{c}
\dot{\boldsymbol{u}} \\
\dot{\boldsymbol{v}} \\
\dot{\boldsymbol{\kappa}}
\end{array}\right)=\left[\begin{array}{ccc}
\mathbf{0} & \boldsymbol{I} & \mathbf{0} \\
-\boldsymbol{M}^{-1} \mathcal{K}_{e} & \mathbf{0} & \boldsymbol{M}^{-1} \mathcal{D}^{a} \boldsymbol{C}_{e} \boldsymbol{\Omega} \\
\boldsymbol{\Lambda}_{e} \mathcal{D} & \mathbf{0} & -\boldsymbol{\Lambda}_{R}
\end{array}\right]\left(\begin{array}{c}
\boldsymbol{u} \\
\boldsymbol{v} \\
\boldsymbol{\kappa}
\end{array}\right)+\left(\begin{array}{c}
\mathbf{0} \\
\boldsymbol{M}^{-1} \boldsymbol{f}(\boldsymbol{x}, t) \\
\mathbf{0}
\end{array}\right)
$$

where $\mathcal{K}_{e}:=\mathcal{D}^{a} C_{e} \mathcal{D}$ is the elastic stiffness operator. They are a set of equations in $N$ scalar displacement fields $\boldsymbol{u}, N$ scalar velocities $\boldsymbol{v}$, and $M$ internal variables $\boldsymbol{\kappa}$. System (3) must be integrated with boundary conditions and the initial conditions (assuming the system is initially at rest)

$$
\boldsymbol{u}(0)=\mathbf{0}, \quad \boldsymbol{v}(0)=\mathbf{0}, \quad \boldsymbol{\kappa}(0)=\mathbf{0} .
$$

\section{Subspace reduction}

The main hypothesis that all the viscous moduli of the structure are small is introduced so that the system is weakly damped. This smallness is accounted for by letting $\mathscr{O}\left(\left|\boldsymbol{\Lambda}_{\vartheta}\right|\right)=\epsilon^{-1}$, with $\epsilon$ a small perturbation parameter $(\vartheta=e, R)$. The existence of this parameter in the flow law makes the equations of motion singularly perturbed (since a small parameter affects the highest derivative [Fusco and Hale 1989; Nayfeh 2000]). It is easy to check, for example via a perturbation method [Luongo and D'Annibale 2017a], that such systems admit a well separated set of eigenvalues: (a) strongly damped real eigenvalues, on the order of $\epsilon^{-1}$, and (b) weakly damped complex conjugate eigenvalues, whose negative real part is of order $\epsilon$. The real eigenvalues are responsible for fast decaying motions and the complex eigenvalues for weakly decaying oscillatory motions. When $\epsilon$ decreases towards zero, the real eigenvalues move to the left side of the complex plane, making the decaying motion faster; the complex eigenvalues instead approach the imaginary axis, rendering the oscillatory motion more weakly decaying. The contrary, of course, occurs, when $\epsilon$ increases from zero. ${ }^{1}$

It is important to underline the fact that such a well separated spectrum can be recognized in viscoelastic structures when the order of magnitude of the relaxation time of the viscoelastic material is smaller than the natural periods of the structure.

\footnotetext{
${ }^{1}$ Note that this occurrence is completely different from the classical static phenomena of creep and relaxation tests, performed, e.g., on concrete and steel specimens. In those cases, indeed, damping is so strong that the real eigenvalues are closer to the imaginary axis than the complex eigenvalues, so that oscillations are fast damped, and the evolution is slow and quasistatic, driven by the real eigenvalues.
} 
This requirement cannot be met by most structural materials, since the behavior of a viscoelastic material with such a small relaxation time is close to that of a fluid. This demand could instead be satisfied, e.g., (i) in multilayered composites, (ii) in structures equipped with viscoelastic suspensions, or with fluid dampers (see, e.g., [Muscolino and Palmeri 2007]), or (iii) in homogenized microstructured viscoelastic systems and metamaterials, where specifically damping properties are designed (see, e.g., [Lekszycki et al. 1992; Manimala and Sun 2014; Del Vescovo and Giorgio 2014; Altenbach and Eremeyev 2015; Frazier and Hussein 2015; Giorgio et al. 2017; Lewińska et al. 2017; Barchiesi et al. 2019; Eugster et al. 2019]). In the Appendix, a one-dimensional example, i.e., a homogenized microstructured continuum, whose periodic microstructure is composed by standard three-parameter oscillators, sheds light on these aspects and permits discussing them numerically. All the sample systems, for which the subspace reduction will be carried out in the next sections, should be thought of as belonging to the categories mentioned above.

Coming back to slightly damped systems, it can be noticed that, if the transient dynamics related to the decaying eigenvalues is ignored, motions occur in a subspace spanned by the eigenvectors associated with the complex eigenvalues. This space, indeed, is much smaller than the original state space, so it is convenient to derive a reduced order model able to describe these dynamics. To construct the model, the space must be contracted without, of course, evaluating all the eigenvectors of the subspace; this is, indeed, possible, as will be illustrated soon.

Two alternative methods are worked out to achieve the goal, both of them borrowed from bifurcation theory, where they are commonly used to deal with nonlinear systems, namely (a) the center manifold method, and (b) the multiple scales method. The first one is a direct (but not trivial) generalization of the algorithm developed in [Luongo and D'Annibale 2017a] for discrete systems.

Center manifold method. According to the center manifold method, the eigenvalues are separated in the set of the stable eigenvalues (leftmost) and in the set of the central eigenvalues (close to the left side of imaginary axis). The associated eigenvectors span the stable and the central subspaces. The center manifold theorem [Guckenheimer and Holmes 1983; Wiggins 2003; Troger and Steindl 1991] assures that there exists an invariant manifold of the same dimensions as the central subspace $\pi$ that contains the origin, is tangent to the subspace $\pi$, and is attractive for the dynamics. Of course, since the system under study is linear, the manifold coincides with the subspace itself. However, differently from the hypotheses of the theorem, the center subspace is infinite-dimensional; in spite of this, the method is heuristically applied.

The Cartesian equations of the unknown subspace $\pi$ are

$$
\kappa=\mathcal{P} \boldsymbol{u}+\mathcal{Q} v
$$


where $\mathcal{P}$ and $\mathcal{Q}$ are unknown differential operators. If these operators are known, the first two equations of motion (3) 1,2 can be rewritten as

$$
\left(\begin{array}{c}
\dot{\boldsymbol{u}} \\
\dot{\boldsymbol{v}}
\end{array}\right)=\left[\begin{array}{cc}
\mathbf{0} & \boldsymbol{I} \\
-\boldsymbol{M}^{-1}\left(\mathcal{K}_{e}-\mathcal{D}^{a} \boldsymbol{C}_{e} \boldsymbol{\Omega} \mathcal{P}\right) & \boldsymbol{M}^{-1} \mathcal{D}^{a} \boldsymbol{C}_{e} \boldsymbol{\Omega \mathcal { Q }}
\end{array}\right]\left(\begin{array}{l}
\boldsymbol{u} \\
\boldsymbol{v}
\end{array}\right)+\left(\begin{array}{c}
\mathbf{0} \\
\boldsymbol{M}^{-1} \boldsymbol{f}
\end{array}\right)
$$

or

$$
M \ddot{\boldsymbol{u}}+\mathcal{B}_{r} \dot{\boldsymbol{u}}+\mathcal{K}_{r} \boldsymbol{u}=\boldsymbol{f}
$$

where

$$
\begin{aligned}
& \mathcal{B}_{r}:=-\mathcal{D}^{a} \boldsymbol{C}_{e} \boldsymbol{\Omega} \mathcal{Q}, \\
& \mathcal{K}_{r}:=\mathcal{K}_{e}-\mathcal{D}^{a} \boldsymbol{C}_{e} \boldsymbol{\Omega} \mathcal{P} .
\end{aligned}
$$

Equation (7) governs the linear dynamics of the system, reduced to the central subspace.

The task, therefore, is to determine $\mathcal{P}$ and $\mathcal{Q}$. This is accomplished by substituting (5) in the flow law $(3)_{3}$, with the help of $(3)_{1,2}$, and requiring it to be satisfied separately for independent $\boldsymbol{u}, \boldsymbol{v}$. It follows that

$$
\begin{aligned}
\left(-\mathcal{Q} M^{-1} \mathcal{K}_{e}+\mathcal{Q} M^{-1} \mathcal{D}^{a} \boldsymbol{C}_{e} \boldsymbol{\Omega} \mathcal{P}-\boldsymbol{\Lambda}_{e} \mathcal{D}+\boldsymbol{\Lambda}_{R} \mathcal{P}\right) \boldsymbol{u} & =\mathbf{0} \\
\left(\mathcal{P}+\mathcal{Q} \boldsymbol{M}^{-1} \mathcal{D}^{a} \boldsymbol{C}_{e} \boldsymbol{\Omega} \mathcal{Q}+\boldsymbol{\Lambda}_{R} \mathcal{Q}\right) \boldsymbol{v} & =\mathbf{0}
\end{aligned}
$$

In order to solve these nonlinear equations, a perturbation method is developed here. The viscous moduli are rescaled at the $\epsilon^{-1}$-order, so that $\boldsymbol{\Lambda}_{\vartheta} \rightarrow \epsilon^{-1} \boldsymbol{\Lambda}_{\vartheta}$ $(\vartheta=e, R)$ and, by multiplying the equations by $\epsilon$,

$$
\begin{array}{r}
{\left[\boldsymbol{\Lambda}_{R} \mathcal{P}-\boldsymbol{\Lambda}_{e} \mathcal{D}-\epsilon \mathcal{Q} M^{-1}\left(\mathcal{K}_{e}-\mathcal{D}^{a} C_{e} \boldsymbol{\Omega} \mathcal{P}\right)\right] \boldsymbol{u}=\mathbf{0},} \\
{\left[\boldsymbol{\Lambda}_{R} \mathcal{Q}+\epsilon\left(\mathcal{P}+\mathcal{Q} \boldsymbol{M}^{-1} \mathcal{D}^{a} \boldsymbol{C}_{e} \boldsymbol{\Omega} \mathcal{Q}\right)\right] \boldsymbol{v}=\mathbf{0} .}
\end{array}
$$

An inspection of these latter equations reveals that $\mathcal{P}(-\epsilon)=\mathcal{P}(\epsilon)$, while $\mathcal{Q}(-\epsilon)=$ $-\mathcal{Q}(\epsilon)$. Thus, the unknown matrices are expanded in series of even or odd powers of $\epsilon$, respectively:

$$
\begin{aligned}
& \mathcal{P}=\mathcal{P}_{0}+\epsilon^{2} \mathcal{P}_{2}+\cdots, \\
& \mathcal{Q}=\epsilon \mathcal{Q}_{1}+\epsilon^{3} \mathcal{Q}_{3}+\cdots,
\end{aligned}
$$

where $\mathcal{P}_{k}=\left.\frac{1}{k !} \frac{\mathrm{d}^{k} \mathcal{P}}{\mathrm{d} \epsilon^{k}}\right|_{\epsilon=0}$, with $k=0,2, \ldots$, and $\mathcal{Q}_{k}=\left.\frac{1}{k !} \frac{\mathrm{d}^{k} \mathcal{Q}}{\mathrm{d} \epsilon^{k}}\right|_{\epsilon=0}$, with $k=1,3, \ldots$ The following linear perturbation equations are then obtained:

$$
\begin{array}{rlrl}
\epsilon^{0}: & & \left(\boldsymbol{\Lambda}_{R} \mathcal{P}_{0}-\boldsymbol{\Lambda}_{e} \mathcal{D}\right) \boldsymbol{u} & =\mathbf{0}, \\
\epsilon^{1}: & \left(\boldsymbol{\Lambda}_{R} \mathcal{Q}_{1}+\mathcal{P}_{0}\right) \boldsymbol{v} & =\mathbf{0}, \\
\epsilon^{2}: & \left(\boldsymbol{\Lambda}_{R} \mathcal{P}_{2}-\mathcal{Q}_{1} \boldsymbol{M}^{-1}\left(\mathcal{K}_{e}-\mathcal{D}^{a} \boldsymbol{C}_{e} \boldsymbol{\Omega} \mathcal{P}_{0}\right)\right) \boldsymbol{u} & =\mathbf{0} .
\end{array}
$$


By solving them in cascade, it is found that

$$
\begin{aligned}
& \mathcal{P}_{0}=\boldsymbol{\Lambda}_{R}^{-1} \boldsymbol{\Lambda}_{e} \mathcal{D} \\
& \mathcal{Q}_{1}=-\boldsymbol{\Lambda}_{R}^{-2} \boldsymbol{\Lambda}_{e} \mathcal{D}, \\
& \mathcal{P}_{2}=-\boldsymbol{\Lambda}_{R}^{-3} \boldsymbol{\Lambda}_{e} \mathcal{D} \boldsymbol{M}^{-1}\left(\mathcal{K}_{e}-\mathcal{D}^{a} \boldsymbol{C}_{e} \boldsymbol{\Omega} \boldsymbol{\Lambda}_{R}^{-1} \boldsymbol{\Lambda}_{e} \mathcal{D}\right) .
\end{aligned}
$$

Finally, by reabsorbing the perturbation parameter, it follows that

$$
\begin{aligned}
& \mathcal{P}=\boldsymbol{\Lambda}_{R}^{-1} \boldsymbol{\Lambda}_{e} \mathcal{D}-\boldsymbol{\Lambda}_{R}^{-3} \boldsymbol{\Lambda}_{e} \mathcal{D} \boldsymbol{M}^{-1}\left(\mathcal{K}_{e}-\mathcal{D}^{a} \boldsymbol{C}_{e} \boldsymbol{\Omega} \boldsymbol{\Lambda}_{R}^{-1} \boldsymbol{\Lambda}_{e} \mathcal{D}\right)+\cdots, \\
& \mathcal{Q}=-\boldsymbol{\Lambda}_{R}^{-2} \boldsymbol{\Lambda}_{e} \mathcal{D}+\cdots
\end{aligned}
$$

Equations (14) are explicit relations that, once substituted in (8), permit us to build the reduced system (7). By truncating the analysis at the first nonzero term,

$$
\begin{aligned}
\mathcal{B}_{r} & :=\mathcal{D}^{a} \boldsymbol{C}_{e} \boldsymbol{\Omega} \boldsymbol{\Lambda}_{R}^{-2} \boldsymbol{\Lambda}_{e} \mathcal{D}, \\
\mathcal{K}_{r} & :=\mathcal{K}_{e}-\mathcal{D}^{a} \boldsymbol{C}_{e} \boldsymbol{\Omega} \boldsymbol{\Lambda}_{R}^{-1} \boldsymbol{\Lambda}_{e} \mathcal{D} .
\end{aligned}
$$

If, instead, an additional term is kept in the expansion of $\mathcal{P}$, an increment of stiffness must be added, so that $\mathcal{K}_{r}^{\mathrm{II}}:=\mathcal{K}_{r}+\Delta \mathcal{K}_{r}$, with

$$
\Delta \mathcal{K}_{r}=\mathcal{D}^{a} \boldsymbol{C}_{e} \boldsymbol{\Omega} \boldsymbol{\Lambda}_{R}^{-3} \boldsymbol{\Lambda}_{e} \mathcal{D} \boldsymbol{M}^{-1} \mathcal{K}_{r} .
$$

The first-order expressions (15) suggest two considerations. First, the damping matrix is a linear combination of the same differential operators which form the elastic (as well as the reduced) stiffness operator. However, due to the structure of the relaxation matrices, the coefficients of the linear combinations are different for the two matrices. Only under special circumstances does it occur that $\mathcal{B}_{r}$ and $\mathcal{K}_{r}$ are proportional; in general, the Rayleigh model of damping is not recovered.

As a second remark, the stiffness $\mathcal{K}_{r}$ has an important physical meaning. Indeed, if reference is made to a relaxation test, at the time $t=\infty$, it is $\dot{\boldsymbol{\kappa}}_{\infty}=0$; therefore, from the flow law (3) $)_{3}$, it follows that $\boldsymbol{\Lambda}_{R} \kappa_{\infty}=\boldsymbol{\Lambda}_{e} \mathcal{D} \boldsymbol{u}$. Hence, from (15)

$$
\mathcal{K}_{r} \boldsymbol{u}=\mathcal{D}^{a} \boldsymbol{C}_{e} \mathcal{D} \boldsymbol{u}-\mathcal{D}^{a} \boldsymbol{C}_{e} \boldsymbol{\Omega} \boldsymbol{\Lambda}_{R}^{-1} \boldsymbol{\Lambda}_{e} \mathcal{D} \boldsymbol{u}=\mathcal{D}^{a} \boldsymbol{C}_{e}\left(\boldsymbol{e}-\boldsymbol{\Omega} \boldsymbol{\kappa}_{\infty}\right)=\mathcal{D}^{a} \boldsymbol{s}_{\infty}
$$

where the elastic law (2) $)_{1}$ is used. By defining an elastic matrix at the equilibrium $\boldsymbol{C}_{e \infty}$, such that $\boldsymbol{C}_{e \infty} \boldsymbol{e}=\boldsymbol{s}_{\infty}$, it is concluded that

$$
\mathcal{K}_{r}=\mathcal{D}^{a} \boldsymbol{C}_{e \infty} \mathcal{D}=: \mathcal{K}_{e \infty},
$$

i.e., at the first order of the asymptotic expansion, the stiffness operator of the reduced system coincides with the elastic matrix, but with the elastic constants replaced by the equilibrium values of the relaxation moduli. This result is not surprising, since a weakly damped system is under consideration, for which the relaxation time is short and of the same order as the transient dynamics. Thus, 
when steady-state dynamics takes place on the central subspace, the relaxation moduli have attained their equilibrium values.

Multiple scales method. Now an alternative approach, based on the multiple scales method [Nayfeh 2000; Nayfeh and Mook 1979; Luongo and Zulli 2012a; 2012b; 2014; Luongo and D'Annibale 2013; 2017b; Luongo et al. 2016], is discussed. The flow law $(3)_{3}$ is rewritten with damping rescaled:

$$
\epsilon \dot{\boldsymbol{\kappa}}=\boldsymbol{\Lambda}_{e} \mathcal{D} \boldsymbol{u}-\boldsymbol{\Lambda}_{R} \boldsymbol{\kappa} .
$$

This is a singularly perturbed differential equation in which $\boldsymbol{u}$ (the active variable) is taken as known, and $\boldsymbol{\kappa}$ (the passive variable) as unknown. Although this equation is linear, with constant coefficients, the multiple scales method is applied to solve it, in order to get an asymptotic solution, useful to the reduction process.

First, several time scales $t_{k}:=\epsilon^{k} t$ with $k=0,1,2, \ldots$ are introduced and it assumed that $\boldsymbol{u}(\boldsymbol{x}, t)=\boldsymbol{u}\left(\boldsymbol{x}, t_{0}, t_{1}, \ldots\right)$ and $\boldsymbol{\kappa}(\boldsymbol{x}, t)=\boldsymbol{\kappa}\left(\boldsymbol{x}, t_{0}, t_{1}, \ldots\right)$; consequently, $\frac{\mathrm{d}}{\mathrm{d} t}=\partial_{t_{0}}+\epsilon \partial_{t_{1}}+\cdots$. Second, the unknown is expanded as

$$
\kappa=\kappa_{0}+\epsilon \kappa_{1}+\epsilon^{2} \kappa_{2}+\cdots
$$

where $\boldsymbol{\kappa}_{k}=\left.\frac{1}{k !} \frac{\mathrm{d}^{k} \boldsymbol{\kappa}_{k}}{\mathrm{~d} \epsilon^{k}}\right|_{\epsilon=0}$, with $k=0,1,2, \ldots$ The following perturbation equations are derived:

$$
\begin{array}{ll}
\epsilon^{0}: & \boldsymbol{\Lambda}_{R} \boldsymbol{\kappa}_{0}=\boldsymbol{\Lambda}_{e} \mathcal{D} \boldsymbol{u}, \\
\epsilon^{1}: & \boldsymbol{\Lambda}_{R} \boldsymbol{\kappa}_{1}=-\partial_{t_{0}} \boldsymbol{\kappa}_{0}, \\
\epsilon^{2}: & \boldsymbol{\Lambda}_{R} \boldsymbol{\kappa}_{2}=-\partial_{t_{0}} \boldsymbol{\kappa}_{1}-\partial_{t_{1}} \boldsymbol{\kappa}_{0} .
\end{array}
$$

The singular nature of the problem is encompassed by the fact that the generating, lower-order equation is not a differential equation in time. By solving the equations in cascade, it follows that

$$
\begin{aligned}
\boldsymbol{\kappa}_{0} & =\boldsymbol{\Lambda}_{R}^{-1} \boldsymbol{\Lambda}_{e} \mathcal{D} \boldsymbol{u}, \\
\boldsymbol{\kappa}_{1} & =-\boldsymbol{\Lambda}_{R}^{-2} \boldsymbol{\Lambda}_{e} \mathcal{D} \partial_{t_{0}} \boldsymbol{u}, \\
\boldsymbol{\kappa}_{2} & =\boldsymbol{\Lambda}_{R}^{-3} \boldsymbol{\Lambda}_{e} \mathcal{D} \partial_{t_{0}}^{2} \boldsymbol{u}-\boldsymbol{\Lambda}_{R}^{-2} \boldsymbol{\Lambda}_{e} \mathcal{D} \partial_{t_{1}} \boldsymbol{u} .
\end{aligned}
$$

By coming back to the series for $\kappa$, reabsorbing the perturbation parameter, and applying the "reconstitution principle" [Nayfeh 2000; Nayfeh and Mook 1979; Luongo and Paolone 1999], it is

$$
\boldsymbol{\kappa}=\boldsymbol{\Lambda}_{R}^{-1} \boldsymbol{\Lambda}_{e} \mathcal{D} \boldsymbol{u}-\boldsymbol{\Lambda}_{R}^{-2} \boldsymbol{\Lambda}_{e} \mathcal{D} \dot{\boldsymbol{u}}+\boldsymbol{\Lambda}_{R}^{-3} \boldsymbol{\Lambda}_{e} \mathcal{D} \ddot{\boldsymbol{u}}+\cdots,
$$

having taken into account that $\dot{\boldsymbol{u}}=\partial_{t_{0}} \boldsymbol{u}+\epsilon \partial_{t_{1}} \boldsymbol{u}+\cdots$ and $\ddot{\boldsymbol{u}}=\partial_{t_{0}}^{2} \boldsymbol{u}+\cdots$ and, consistently, having truncated the expansions. 
By substituting this latter expression in the equations of motion $(3)_{1,2}$ the reduced model is finally obtained as

$\left(\boldsymbol{M}-\mathcal{D}^{a} \boldsymbol{C}_{e} \boldsymbol{\Omega} \boldsymbol{\Lambda}_{R}^{-3} \boldsymbol{\Lambda}_{e} \mathcal{D}\right) \ddot{\boldsymbol{u}}+\left(\mathcal{D}^{a} \boldsymbol{C}_{e} \boldsymbol{\Omega} \boldsymbol{\Lambda}_{R}^{-2} \boldsymbol{\Lambda}_{e} \mathcal{D}\right) \dot{\boldsymbol{u}}+\left(\mathcal{K}_{e}-\mathcal{D}^{a} \boldsymbol{C}_{e} \boldsymbol{\Omega} \boldsymbol{\Lambda}_{R}^{-1} \boldsymbol{\Lambda}_{e} \mathcal{D}\right) \boldsymbol{u}=\boldsymbol{f}$.

In this equation, the stiffness and the damping operators are identical to those derived by the center manifold approach (see (15)). Moreover, a reduced mass (differential) operator appears, which was absent in the previous derivation. However, if the lower-order approximation (rendered homogeneous) is taken for $\ddot{\boldsymbol{u}}$, namely $\ddot{\boldsymbol{u}}=-\boldsymbol{M}^{-1} \mathcal{K}_{r} \boldsymbol{u}$, it is found that this extra term is consistent with the increment of stiffness (16). Therefore, the two approach are asymptotically equivalent.

The algorithm illustrated here better underlines the sequence of approximations, which is able to shed light on the physics of the systems. First, the internal variables are linked to the observable variables as $\dot{\boldsymbol{e}}_{v}=\mathbf{0}$, this approximation being sufficient to determine the reduced stiffness with the equilibrium relaxation moduli. Second, the resultant expression for $\boldsymbol{e}_{v}$ supplies the strain rate $\dot{\boldsymbol{e}}_{v}$, from which the damping is evaluated. Third, a better approximation for $\dot{\boldsymbol{e}}_{v}$ is computed that modifies the mass or, equivalently, the stiffness.

Throughout the following working examples, the second-order approximation is neglected.

\section{Viscoelastic Euler-Bernoulli beam on Winkler viscoelastic soil}

Model. The transverse motion of a shear-undeformable planar beam resting on viscoelastic soil is considered (Figure 2). Kinematics is described by

$$
\chi=u^{\prime \prime}, \quad \varepsilon=u
$$

where $u(s, t)$ is the deflection of the beam, $\chi(s, t)$ its curvature, and $\varepsilon(s, t)$ the elongation of soil, with $s$ the abscissa, $t$ the time, and a prime denoting $s$-differentiation.

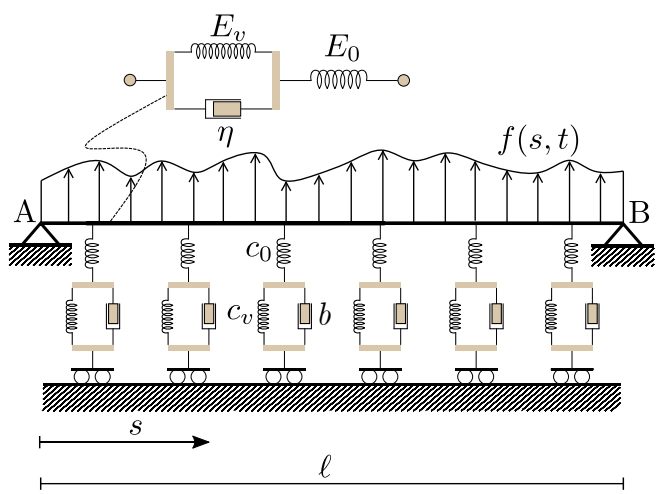

Figure 2. Planar viscoelastic beam on viscoelastic soil. 
Equilibrium entails

$$
M^{\prime \prime}=-r(s, t)-m \ddot{u}+f(s, t)
$$

where $M(s, t)$ is the bending moment, $r(s, t)$ the linear density of the soil reaction, $m$ the mass per unit length of the beam, and $f(s, t)$ the linear density of the external active forces.

The constitutive behavior of the beam and of the soil obeys the viscoelastic standard three-parameter model (Figure 1, left). The constitutive law for the beam is

$$
\begin{aligned}
& M=E_{0} I\left(\chi-\chi_{v}\right), \\
& \dot{\chi}_{v}=\frac{E_{0}}{\eta}\left(\chi-\chi_{v}\right)-\frac{E_{v}}{\eta} \chi_{v}
\end{aligned}
$$

where $E_{0}, E_{v}, \eta$ are material constants, $\chi_{v}$ is an internal variable having the meaning of viscous curvature, and $I$ is the cross-section inertia moment. The constitutive law for the soil is

$$
\begin{aligned}
r & =c_{0}\left(\varepsilon-\varepsilon_{v}\right), \\
\dot{\varepsilon}_{v} & =\frac{c_{0}}{b}\left(\varepsilon-\varepsilon_{v}\right)-\frac{c_{v}}{b} \varepsilon_{v}
\end{aligned}
$$

where $c_{0}, c_{v}, b$ are soil constants and $\varepsilon_{v}$ is an internal variable describing the viscous deformation of the soil. When all these equations are collected, and put in the state form, the viscoelastic problem reads

$$
\begin{aligned}
\dot{u} & =v, \\
m \dot{v}+E_{0} I\left(u^{\prime \prime \prime \prime}-\chi_{v}^{\prime \prime}\right)+c_{0}\left(u-\varepsilon_{v}\right) & =f(s, t), \\
\dot{\chi}_{v} & =\Lambda_{e b} \chi-\Lambda_{R b} \chi_{v}, \\
\dot{\varepsilon}_{v} & =\Lambda_{e s} \varepsilon-\Lambda_{R s} \varepsilon_{v}
\end{aligned}
$$

with $v$ the velocity, and the relaxation coefficients of beam and soil defined as

$$
\Lambda_{e b}:=\frac{E_{0}}{\eta}, \quad \Lambda_{R b}:=\frac{E_{0}+E_{v}}{\eta}, \quad \Lambda_{e s}:=\frac{c_{0}}{b}, \quad \Lambda_{R s}:=\frac{c_{0}+c_{v}}{b} .
$$

It is easy to check that (29) are of the type (3) with the identifications

$$
\begin{aligned}
& \boldsymbol{u}:=[u], \quad \boldsymbol{v}:=[v], \quad \boldsymbol{e}=\left[\begin{array}{l}
\chi \\
\varepsilon
\end{array}\right], \quad \boldsymbol{\kappa}=\left[\begin{array}{l}
\chi_{v} \\
\varepsilon_{v}
\end{array}\right], \\
& \mathcal{D}=\left[\begin{array}{c}
\partial_{s}^{2} \\
1
\end{array}\right], \quad \mathcal{D}^{a}=\left[\begin{array}{ll}
\partial_{s}^{2} & 1
\end{array}\right], \quad \boldsymbol{M}:=[m], \quad \boldsymbol{C}_{e}=\left[\begin{array}{cc}
E_{0} I & 0 \\
0 & c_{0}
\end{array}\right], \\
& \mathcal{K}_{e}=\left[E_{0} I \partial_{s}^{4}+c_{0}\right], \boldsymbol{\Lambda}_{e}=\left[\begin{array}{cc}
\Lambda_{e b} & 0 \\
0 & \Lambda_{e s}
\end{array}\right], \boldsymbol{\Lambda}_{R}=\left[\begin{array}{cc}
\Lambda_{R b} & 0 \\
0 & \Lambda_{R s}
\end{array}\right], \quad \boldsymbol{\Omega}=\left[\begin{array}{ll}
1 & 0 \\
0 & 1
\end{array}\right]
\end{aligned}
$$

where $\partial_{s}^{k}:=\frac{\partial^{k}}{\partial s^{k}}$. 
Equations (29) must be constrained by boundary conditions. For example, if the beam is simply supported at the ends A, B, boundary conditions read

$$
u_{H}=0, \quad E_{0} I\left(u_{H}^{\prime \prime}-\chi_{v H}\right)=0, \quad H=\mathrm{A}, \mathrm{B} .
$$

Reduced system. The reduction process requires linearly linking the internal variables to the observable variables via unknown differential operators $P_{\vartheta}$ and $Q_{\vartheta}$ $(\vartheta=b, s)($ remember $(5))$ :

$$
\left(\begin{array}{l}
\chi_{v} \\
\varepsilon_{v}
\end{array}\right)=\left[\begin{array}{l}
P_{b} \\
P_{s}
\end{array}\right] u+\left[\begin{array}{l}
Q_{b} \\
Q_{s}
\end{array}\right] v=\mathcal{P} \boldsymbol{u}+\mathcal{Q} v .
$$

By using (14) and the definitions (31), truncated at the first term, it is found that

$$
\mathcal{P}=\left[\begin{array}{c}
\left(\Lambda_{e b} / \Lambda_{R b}\right) \partial_{s}^{2} \\
\Lambda_{e s} / \Lambda_{R s}
\end{array}\right], \quad \mathcal{Q}=-\left[\begin{array}{c}
\left(\Lambda_{e b} / \Lambda_{R b}^{2}\right) \partial_{s}^{2} \\
\Lambda_{e s} / \Lambda_{R s}^{2}
\end{array}\right]
$$

so that the operators are mixed, differential-algebraic. With these, from (8), or (15), the stiffness and damping of the reduced system are found, namely

$$
\begin{aligned}
\mathcal{B}_{r} & =\left[E_{0} I \frac{\Lambda_{e b}}{\Lambda_{R b}^{2}} \partial_{s}^{4}+c_{0} \frac{\Lambda_{e s}}{\Lambda_{R s}^{2}}\right], \\
\mathcal{K}_{r} & =\left[E_{0} I\left(1-\frac{\Lambda_{e b}}{\Lambda_{R b}}\right) \partial_{s}^{4}+c_{0}\left(1-\frac{\Lambda_{e s}}{\Lambda_{R s}}\right)\right] .
\end{aligned}
$$

Finally, the reduced equation of motion (7), in terms of only observable variables, reads

$$
m \ddot{u}+\alpha I \dot{u}^{\prime \prime \prime \prime}+b^{\star} \dot{u}+E_{\infty} I u^{\prime \prime \prime \prime \prime}+c_{\infty} u=f
$$

where, by accounting for the definitions (30), the following viscoelastic coefficients have been introduced:

$$
\alpha:=\eta\left(\frac{E_{0}}{E_{0}+E_{v}}\right)^{2}, \quad b^{\star}:=b\left(\frac{c_{0}}{c_{0}+c_{v}}\right)^{2}, \quad E_{\infty}:=\frac{E_{0} E_{v}}{E_{0}+E_{v}}, \quad c_{\infty}:=\frac{c_{0} c_{v}}{c_{0}+c_{v}} .
$$

Note that this model describes an internally and externally damped beam on a Winkler elastic soil; in particular, $\alpha$ is the internal damping coefficient, depending on the elastic and viscous properties of the beam; $b^{\star}$ is the external damping coefficient, which depends on the elastic and viscous properties of the soil; and $E_{\infty}, c_{\infty}$ are the relaxation moduli at the equilibrium. It is worth noticing that, in this model, damping is not proportional to the stiffness.

The internal damping of the beam. When the beam is in the air (i.e., not resting on soil, so $c_{0}=c_{v}=0$ ) the reduced model (36), in the absence of external forces, becomes

$$
m \ddot{u}+\eta\left(\frac{E_{0}}{E_{0}+E_{v}}\right)^{2} I \dot{u}^{\prime \prime \prime \prime \prime}+E_{\infty} I u^{\prime \prime \prime \prime \prime}=0 .
$$


Let $\phi_{j}(s)$ be the $j$-th natural mode of the elastic beam, of frequency $\omega_{j}$, satisfying

$$
E_{\infty} I \phi_{j}^{\prime \prime \prime \prime}-m \omega_{j}^{2} \phi_{j}=0
$$

with the relevant boundary conditions. Since damping is now of proportional type, $\phi_{j}$ is also an eigenvector for the viscoelastic beam. Thus, by substituting $u(s, t)=x_{j}(t) \phi_{j}(s)$ in the equation of motion, and accounting for (39), an ordinary differential equation for the modal amplitude $x_{j}(t)$ is obtained, namely

$$
\ddot{x}_{j}+\eta \omega_{j}^{2} \frac{E_{\infty}}{E_{v}^{2}} \dot{x}_{j}+\omega_{j}^{2} x_{j}=0 .
$$

With the usual definition of damping factor, and by using $(37)_{3}$ and $(30)_{2}$, it is found that

$$
\xi_{j}=\frac{1}{2} \eta \frac{E_{\infty}}{E_{v}^{2}} \omega_{j} \equiv \frac{1}{2} \frac{E_{0}}{E_{v}} \frac{\omega_{j}}{\Lambda_{R}}
$$

where $\Lambda_{R} \equiv \Lambda_{R b}$ and which, consistently with the Rayleigh model of damping (proportional to the stiffness), is proportional to the undamped natural frequency. This last expression is believed to be useful in damping modeling and design of beams.

\section{Viscoelastic Kirchhoff plate}

Model. The transverse vibrations of a thin viscoelastic plate, modeled according to Kirchhoff's theory, are considered (see Figure 3).

Kinematics is described by

$$
\left(\begin{array}{l}
\chi_{x} \\
\chi_{y} \\
\chi_{x y}
\end{array}\right)=\left[\begin{array}{c}
\partial_{x x}^{2} \\
\partial_{y y}^{2} \\
2 \partial_{x y}^{2}
\end{array}\right](u)
$$

where $u(\boldsymbol{x}, t)$ is the transverse deflection, with $\boldsymbol{x}=x \boldsymbol{a}_{x}+y \boldsymbol{a}_{y}$ the position; $\chi_{x}(\boldsymbol{x}, t)$, $\chi_{y}(\boldsymbol{x}, t)$ are flexural curvatures and $\chi_{x y}(\boldsymbol{x}, t)$ the torsional curvature; and finally

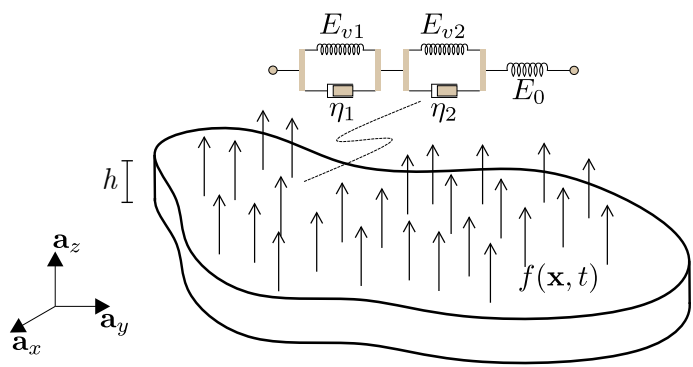

Figure 3. Viscoelastic Kirchhoff plate. 
$\partial_{\vartheta_{1} \cdots \vartheta_{k}}^{k}\left(\vartheta_{i}=x, y\right)$ denotes the partial derivative of order $k$ with respect the homonymous variables.

Equilibrium is ruled by

$$
\left[\partial_{x x}^{2}, \partial_{y y}^{2}, 2 \partial_{x y}^{2}\right]\left(\begin{array}{c}
M_{x} \\
M_{y} \\
M_{x y}
\end{array}\right)=f-\rho \ddot{u}
$$

where $M_{x}, M_{y}$ are bending moments and $M_{x y}$ the torsional moment; moreover, $f(\boldsymbol{x}, t)$ is the surface load density, and $\rho$ the surface mass density.

The constitutive law of the plate is assumed to be governed by the five-parameter model, which is made of one spring $E_{0}$, placed in series with two Kelvin-Voigt systems of constants $E_{v i}, \eta_{i}(i=1,2)$ (Figure 1, right). Moreover, it is also admitted that all the stress components could evolve by the same law with strain components (thus entailing that the deviatoric and spherical stresses of the underlying three-dimensional model have the same viscoelastic evolution). Under these assumptions, the elastic law is

$$
\left(\begin{array}{c}
M_{x} \\
M_{y} \\
M_{x y}
\end{array}\right)=D_{0}\left[\begin{array}{ccc}
1 & v & 0 \\
v & 1 & 0 \\
0 & 0 & (1-v) / 2
\end{array}\right]\left(\begin{array}{c}
\chi_{x}-\kappa_{x 1}-\kappa_{x 2} \\
\chi_{y}-\kappa_{y 1}-\kappa_{y 2} \\
\chi_{x y}-\kappa_{x y 1}-\kappa_{x y 2}
\end{array}\right)
$$

where $\kappa_{\vartheta i}(\vartheta=x, y, x y$ and $i=1,2)$ are the viscous curvatures, relevant to the $i$-th subcomponent of the rheological model, and $D_{0}:=\frac{1}{12} h^{3} E_{0} /\left(1-v^{2}\right)$ is the bending stiffness, with $E_{0}, v$ the Young modulus and the Poisson ratio, and $h$ the plate thickness.

The flow laws, governing the evolution of the internal variables $\kappa_{\vartheta i}$, are

$$
\begin{array}{r}
\left(\begin{array}{l}
\dot{\kappa}_{\vartheta 1} \\
\dot{\kappa}_{\vartheta 2}
\end{array}\right)=\left[\begin{array}{l}
E_{0} / \eta_{1} \\
E_{0} / \eta_{2}
\end{array}\right]\left(\chi_{\vartheta}\right)-\left[\begin{array}{cc}
\left(E_{0}+E_{v 1}\right) / \eta_{1} & E_{0} / \eta_{1} \\
E_{0} / \eta_{2} & \left(E_{0}+E_{v 2}\right) / \eta_{2}
\end{array}\right] \\
\left.\vartheta \begin{array}{l}
\kappa_{\vartheta 1} \\
\kappa_{\vartheta 2}
\end{array}\right), \\
\vartheta=x, y, x y .
\end{array}
$$

All these equations are in the form (1)-(2) and, therefore, implicitly define (i) the differential operators $\mathcal{D}, \mathcal{D}^{a}$ in (42)-(43), (ii) the topological matrix $\boldsymbol{\Omega}$, linking the internal variables, i.e., the viscous curvatures $\kappa_{\vartheta i}$, to the viscous strains (remember $(2)_{2}$ ), and (iii) the elastic matrix $\boldsymbol{C}_{\boldsymbol{e}}$. They read

$$
\begin{array}{rlrl}
\mathcal{D}:=\left[\begin{array}{c}
\partial_{x x}^{2} \\
\partial_{y y}^{2} \\
2 \partial_{x y}^{2}
\end{array}\right], & & \mathcal{D}^{a}:=\left[\partial_{x x}^{2}, \partial_{y y}^{2}, 2 \partial_{x y}^{2}\right], \\
\boldsymbol{\Omega}:=\left[\begin{array}{llllll}
1 & 1 & 0 & 0 & 0 & 0 \\
0 & 0 & 1 & 1 & 0 & 0 \\
0 & 0 & 0 & 0 & 1 & 1
\end{array}\right], & \boldsymbol{C}_{e}:=D_{0}\left[\begin{array}{ccc}
1 & v & 0 \\
v & 1 & 0 \\
0 & 0 & (1-v) / 2
\end{array}\right] .
\end{array}
$$


Moreover, from (45) the definition of $\boldsymbol{\Lambda}_{e}, \boldsymbol{\Lambda}_{R}$ follows, namely

$$
\begin{aligned}
\boldsymbol{\Lambda}_{e}:= & {\left[\begin{array}{ccc}
E_{0} / \eta_{1} & 0 & 0 \\
E_{0} / \eta_{2} & 0 & 0 \\
0 & E_{0} / \eta_{1} & 0 \\
0 & E_{0} / \eta_{2} & 0 \\
0 & 0 & E_{0} / \eta_{1} \\
0 & 0 & E_{0} / \eta_{2}
\end{array}\right], } \\
\boldsymbol{\Lambda}_{R}:= & {\left[\begin{array}{cccccc}
F_{1} & E_{0} / \eta_{1} & 0 & 0 & 0 & 0 \\
E_{0} / \eta_{2} & F_{2} & 0 & 0 & 0 & 0 \\
0 & 0 & F_{1} & E_{0} / \eta_{1} & 0 & 0 \\
0 & 0 & E_{0} / \eta_{2} & F_{2} & 0 & 0 \\
0 & 0 & 0 & 0 & F_{1} & E_{0} / \eta_{1} \\
0 & 0 & 0 & 0 & E_{0} / \eta_{2} & F_{2}
\end{array}\right] }
\end{aligned}
$$

where $F_{j}=\left(E_{0}+E_{v j}\right) / \eta_{j}(j=1,2)$. The equations of motion, when rearranged in the state form (3), are

$$
\dot{w}=\mathcal{A} w+f
$$

where the definitions

$$
\begin{aligned}
& \boldsymbol{w}:=\left(\begin{array}{llllllll}
u & v & \kappa_{x 1} & \kappa_{x 2} & \kappa_{y 1} & \kappa_{y 2} & \kappa_{x y 1} & \kappa_{x y 2}
\end{array}\right)^{T}, \\
& \boldsymbol{f}:=\left(\begin{array}{llllllll}
0 & f / \rho & 0 & 0 & 0 & 0 & 0 & 0
\end{array}\right)^{T}, \\
& \mathcal{A}:=\left[\begin{array}{ccc}
\mathbf{0} & \boldsymbol{I} & \mathbf{0} \\
-(1 / \rho) \mathcal{K}_{e} & \mathbf{0} & (1 / \rho) \mathcal{D}^{a} \boldsymbol{C}_{e} \boldsymbol{\Omega} \\
\boldsymbol{\Lambda}_{e} \mathcal{D} & \mathbf{0} & -\boldsymbol{\Lambda}_{R}
\end{array}\right]
\end{aligned}
$$

hold and in which

$$
\begin{aligned}
\mathcal{K}_{e}:= & D_{0} \nabla^{4} \\
\mathcal{D}^{a} \boldsymbol{C}_{e} \boldsymbol{\Omega}= & D_{0}\left[\partial_{x x}^{2}+v \partial_{y y}^{2} \partial_{x x}^{2}+v \partial_{y y}^{2} \partial_{y y}^{2}+v \partial_{x x}^{2} \partial_{y y}^{2}+v \partial_{x x}^{2}(1-v) \partial_{x y}^{2}(1-v) \partial_{x y}^{2}\right] \\
\boldsymbol{\Lambda}_{e} \mathcal{D}: & :\left(\begin{array}{c}
\left(E_{0} / \eta_{1}\right) \partial_{x x}^{2} \\
\left(E_{0} / \eta_{2}\right) \partial_{x x}^{2} \\
\left(E_{0} / \eta_{1}\right) \partial_{y y}^{2} \\
\left(E_{0} / \eta_{2}\right) \partial_{y y}^{2} \\
2\left(E_{0} / \eta_{1}\right) \partial_{x y}^{2} \\
2\left(E_{0} / \eta_{2}\right) \partial_{x y}^{2}
\end{array}\right) .
\end{aligned}
$$

The dynamics is, therefore, governed by a system of partial differential equations in two observable variables (displacement and velocity) and six internal variables (two viscous components for each of the three curvatures). 
Reduced system. Aimed at reducing the system to the sole observable variables, the internal variables are linked to them via unknown differential operators $\mathcal{P}, \mathcal{Q}$, i.e.,

$$
\left(\begin{array}{c}
\kappa_{x 1} \\
\kappa_{x 2} \\
\kappa_{y 1} \\
\kappa_{y 2} \\
\kappa_{x y 1} \\
\kappa_{x y 2}
\end{array}\right)=\left[\begin{array}{c}
P_{x 1} \\
P_{x 2} \\
P_{y 1} \\
P_{y 2} \\
P_{x y 1} \\
P_{x y 2}
\end{array}\right] u+\left[\begin{array}{l}
Q_{x 1} \\
Q_{x 2} \\
Q_{y 1} \\
Q_{y 2} \\
Q_{x y 1} \\
Q_{x y 2}
\end{array}\right] v=\mathcal{P} \boldsymbol{u}+\mathcal{Q} \boldsymbol{v} .
$$

With the definitions (50) and by using the first-term approximation in (14),

$$
\begin{aligned}
\mathcal{P}=\frac{E_{0}}{E_{0} E_{v 1}+E_{v 1} E_{v 2}+E_{0} E_{v 2}}\left[\begin{array}{c}
E_{v 2} \partial_{x x}^{2} \\
E_{v 1} \partial_{x x}^{2} \\
E_{v 2} \partial_{y y}^{2} \\
E_{v 1} \partial_{y y}^{2} \\
2 E_{v 2} \partial_{x y}^{2} \\
2 E_{v 1} \partial_{x y}^{2}
\end{array}\right], \\
\mathcal{Q}=\frac{E_{0}}{\left(E_{0} E_{v 1}+E_{v 1} E_{v 2}+E_{0} E_{v 2}\right)^{2}}\left[\begin{array}{c}
-\left(E_{0} E_{v 2} \eta_{1}+E_{v 2}^{2} \eta_{1}-E_{0} E_{v 1} \eta_{2}\right) \partial_{x x}^{2} \\
\left(E_{0} E_{v 2} \eta_{1}-E_{v 1}^{2} \eta_{2}-E_{0} E_{v 1} \eta_{2}\right) \partial_{x x}^{2} \\
-\left(E_{0} E_{v 2} \eta_{1}+E_{v 2}^{2} \eta_{1}-E_{0} E_{v 1} \eta_{2}\right) \partial_{y y}^{2} \\
\left(E_{0} E_{v 2} \eta_{1}-E_{v 1}^{2} \eta_{2}-E_{0} E_{v 1} \eta_{2}\right) \partial_{y y}^{2} \\
-2\left(E_{0} E_{v 2} \eta_{1}+E_{v 2}^{2} \eta_{1}-E_{0} E_{v 1} \eta_{2}\right) \partial_{x y}^{2} \\
2\left(E_{0} E_{v 2} \eta_{1}-E_{v 1}^{2} \eta_{2}-E_{0} E_{v 1} \eta_{2}\right) \partial_{x y}^{2}
\end{array}\right]
\end{aligned}
$$

so that the operators (8) of the reduced system are

$$
\mathcal{B}_{r}:=\alpha \nabla^{4}, \quad \mathcal{K}_{r}:=D_{\infty} \nabla^{4}
$$

where the definitions

$$
\alpha:=D_{0} \frac{E_{0}\left(E_{v 2}^{2} \eta_{1}+E_{v 1}^{2} \eta_{2}\right)}{\left(E_{0} E_{v 1}+E_{v 1} E_{v 2}+E_{0} E_{v 2}\right)^{2}}, \quad D_{\infty}:=\frac{h^{3}}{12} \frac{E_{\infty}}{1-v^{2}}
$$

hold, $D_{\infty}$ being the stiffness of the plate at $t=\infty$ with

$$
E_{\infty}:=\frac{E_{0} E_{v 1} E_{v 2}}{E_{0} E_{v 1}+E_{v 1} E_{v 2}+E_{0} E_{v 2}}
$$

the stiffness of the three in-series springs of the five-parameter model.

By summarizing, the reduced model is governed by

$$
D_{\infty} \nabla^{4} u+\alpha \nabla^{4} \dot{u}+\rho \ddot{u}=f .
$$

In this case the damping, governed by the coefficient $\alpha$, is found to be of proportional type, due to the hypotheses made on the constitutive law. 


\section{The Cauchy viscoelastic continuum}

Model. An isotropic Cauchy continuum is considered. The vector of displacements, at point $\boldsymbol{x}$ and time $t$, is denoted by $\boldsymbol{u}(\boldsymbol{x}, t)$, the infinitesimal strain tensor by $\boldsymbol{E}(\boldsymbol{x}, t)$, and the stress tensor by $\boldsymbol{T}(\boldsymbol{x}, t)$. Then the tensors are decomposed in their spherical and deviatoric parts, namely $\boldsymbol{E}=\frac{1}{3} \delta \boldsymbol{I}+\boldsymbol{E}^{\star}$ and $\boldsymbol{T}=\theta \boldsymbol{I}+\boldsymbol{T}^{\star}$, where $\delta:=\operatorname{tr} \boldsymbol{E}$ is the volumetric strain, $\theta=\operatorname{tr} \boldsymbol{T}$ is the hydrostatic stress, $\boldsymbol{I}$ is the identity tensor, and a star denotes the deviatoric part.

Kinematics states that

$$
\delta=\nabla \cdot \boldsymbol{u}, \quad \boldsymbol{E}^{\star}=\frac{1}{2}\left[\nabla \boldsymbol{u}+(\nabla \boldsymbol{u})^{T}\right]-\frac{1}{3}(\boldsymbol{I} \nabla \cdot \boldsymbol{u}) .
$$

Equilibrium calls for

$$
\nabla \cdot\left(\theta \boldsymbol{I}+\boldsymbol{T}^{\star}\right)-\rho \ddot{\boldsymbol{u}}+\boldsymbol{b}=\mathbf{0}
$$

where $\rho$ is the mass density and $\boldsymbol{b}$ the bulk forces.

It is assumed that the body is linear viscoelastic in the deviatoric part, while it is elastic in the spherical part. By adopting the standard three-parameter model, the constitutive law is written as

$$
\begin{aligned}
\boldsymbol{T}^{\star} & =2 \mu\left(\boldsymbol{E}^{\star}-\boldsymbol{E}_{v}^{\star}\right), \\
\dot{\boldsymbol{E}}_{v}^{\star} & =\Lambda_{e} \boldsymbol{E}^{\star}-\Lambda_{R} \boldsymbol{E}_{v}^{\star}, \\
\theta & =K \delta
\end{aligned}
$$

where $\boldsymbol{E}_{v}^{\star}$ is the viscous part and $\boldsymbol{E}^{\star}-\boldsymbol{E}_{v}^{\star}$ the elastic part of the deviatoric strain tensor; $\mu$ and $K:=\lambda+\frac{2}{3} \mu$ are elastic constants, namely the Lamé constants $\lambda, \mu$ and the bulk modulus $K$; and finally, $\Lambda_{e}, \Lambda_{R}$ are relaxation constants.

By arranging the previous equations in the state form (accounting for $\nabla \cdot \nabla \boldsymbol{u}=$ $\nabla^{2} \boldsymbol{u}, \nabla \cdot(\nabla \boldsymbol{u})^{T}=\nabla(\nabla \cdot \boldsymbol{u})$, and $\left.\nabla \cdot(\boldsymbol{I} \nabla \cdot \boldsymbol{u})=\nabla(\nabla \cdot \boldsymbol{u})\right)$, it is

$$
\begin{aligned}
& \dot{\boldsymbol{u}}=\boldsymbol{v}, \\
& \mu \nabla^{2} \boldsymbol{u}+(\mu+\lambda) \nabla(\nabla \cdot \boldsymbol{u})-2 \mu \nabla \cdot \boldsymbol{E}_{v}^{\star}-\rho \dot{\boldsymbol{v}}+\boldsymbol{b}=\mathbf{0}, \\
& \dot{\boldsymbol{E}}_{v}^{\star}=\Lambda_{e}\left[\frac{1}{2} \nabla \boldsymbol{u}+\frac{1}{2}(\nabla \boldsymbol{u})^{T}-\frac{1}{3} \boldsymbol{I} \nabla \cdot \boldsymbol{u}\right]-\Lambda_{R} \boldsymbol{E}_{v}^{\star} .
\end{aligned}
$$

These equations are of the general form (3), although here a different notation is used (vector form, instead of matrix form). When $\boldsymbol{E}_{v}^{\star} \equiv \mathbf{0}$ and the flow law is suppressed, they reduce to the well known Navier equations for the elastic continuum.

Reduced system. To reduce the system to the central subspace, the procedure illustrated for a general system is repeated, by using the vector notation. First, the internal variables are expressed as a linear combination of the displacement and velocity:

$$
\boldsymbol{E}_{v}^{\star}=\mathcal{P} \boldsymbol{u}+\mathcal{Q} \boldsymbol{v}
$$


where $\mathcal{P}, \mathcal{Q}$ are unknown linear differential operators. By differentiating this latter equation with respect to time, using $(60)_{1,2}$ (rendered homogeneous) to eliminate $\dot{\boldsymbol{u}}, \dot{\boldsymbol{v}}$, and similarly using (61) to eliminate $\boldsymbol{E}_{v}^{\star},(60)_{3}$ reads

$$
\begin{aligned}
\mathcal{P} \boldsymbol{v}+\frac{1}{\rho} \mathcal{Q}\left[\mu \nabla^{2} \boldsymbol{u}+(\right. & (\mu+\lambda) \nabla(\nabla \cdot \boldsymbol{u})-2 \mu \nabla \cdot(\mathcal{P} \boldsymbol{u}+\mathcal{Q} \boldsymbol{v})] \\
& =\Lambda_{e}\left[\frac{1}{2} \nabla \boldsymbol{u}+\frac{1}{2}(\nabla \boldsymbol{u})^{T}-\frac{1}{3} \boldsymbol{I} \nabla \cdot \boldsymbol{u}\right]-\Lambda_{R}(\mathcal{P} \boldsymbol{u}+\mathcal{Q} \boldsymbol{v}) .
\end{aligned}
$$

By requiring it holds for any $\boldsymbol{u}, \boldsymbol{v}$, it follows that

$$
\begin{array}{r}
\frac{1}{\rho} \mathcal{Q}\left[\mu \nabla^{2} \boldsymbol{u}+(\mu+\lambda) \nabla(\nabla \cdot \boldsymbol{u})-2 \mu \nabla \cdot(\mathcal{P} \boldsymbol{u})\right]-\Lambda_{e}\left[\frac{1}{2} \nabla \boldsymbol{u}+\frac{1}{2}(\nabla \boldsymbol{u})^{T}-\frac{1}{3} \boldsymbol{I} \nabla \cdot \boldsymbol{u}\right] \\
+\Lambda_{R}(\mathcal{P} \boldsymbol{u})=\mathbf{0}, \\
\mathcal{P} \boldsymbol{v}-\frac{2 \mu}{\rho} \mathcal{Q}[\nabla \cdot(\mathcal{Q} \boldsymbol{v})]+\Lambda_{R} \mathcal{Q} \boldsymbol{v}=\mathbf{0} .
\end{array}
$$

Then, by rescaling $\Lambda_{\vartheta} \rightarrow \epsilon^{-1} \Lambda_{\vartheta}(\vartheta=e, R)$ and expanding the operators as $\mathcal{P}=$ $\mathcal{P}_{0}+\mathcal{O}(\epsilon)$ and $\mathcal{Q}=\epsilon \mathcal{Q}_{1}+\mathcal{O}\left(\epsilon^{2}\right)$, the leading perturbation equations

$$
\begin{aligned}
-\Lambda_{e}\left[\frac{1}{2} \nabla \boldsymbol{u}+\frac{1}{2}(\nabla \boldsymbol{u})^{T}-\frac{1}{3} \boldsymbol{I} \nabla \cdot \boldsymbol{u}\right]+\Lambda_{R}\left(\mathcal{P}_{0} \boldsymbol{u}\right) & =\mathbf{0}, \\
\mathcal{P}_{0} \boldsymbol{v}+\Lambda_{R} \mathcal{Q}_{1} \boldsymbol{v} & =\mathbf{0}
\end{aligned}
$$

are obtained, whose solution is

$$
\begin{aligned}
& \mathcal{P}_{0} \boldsymbol{u}=\frac{1}{2} \frac{\Lambda_{e}}{\Lambda_{R}}\left[\nabla \boldsymbol{u}+(\nabla \boldsymbol{u})^{T}\right]-\frac{1}{3} \frac{\Lambda_{e}}{\Lambda_{R}} \boldsymbol{I} \nabla \cdot \boldsymbol{u}, \\
& \mathcal{Q}_{1} \boldsymbol{v}=-\frac{1}{2} \frac{\Lambda_{e}}{\Lambda_{R}^{2}}\left[\nabla \boldsymbol{v}+(\nabla \boldsymbol{v})^{T}\right]+\frac{1}{3} \frac{\Lambda_{e}}{\Lambda_{R}^{2}} \boldsymbol{I} \nabla \cdot \boldsymbol{v} .
\end{aligned}
$$

Therefore, from (61), at the leading order, it is found that

$$
\boldsymbol{E}_{v}^{\star}=\frac{1}{2} \frac{\Lambda_{e}}{\Lambda_{R}}\left[\nabla \boldsymbol{u}+(\nabla \boldsymbol{u})^{T}\right]-\frac{1}{3} \frac{\Lambda_{e}}{\Lambda_{R}} \boldsymbol{I} \nabla \cdot \boldsymbol{u}-\frac{1}{2} \frac{\Lambda_{e}}{\Lambda_{R}^{2}}\left(\nabla \boldsymbol{v}+\nabla^{T} \boldsymbol{v}\right)+\frac{1}{3} \frac{\Lambda_{e}}{\Lambda_{R}^{2}} \boldsymbol{I} \nabla \cdot \boldsymbol{v},
$$

which permits us to write the balance equations $(60)_{1,2}$ in terms of displacements only:

$$
\begin{array}{r}
\mu_{\infty} \nabla^{2} \boldsymbol{u}+\left[\frac{\mu_{\infty}\left(\Lambda_{e}-3 \Lambda_{R}\right)}{3\left(\Lambda_{e}-\Lambda_{R}\right)}+\lambda\right] \nabla(\nabla \cdot \boldsymbol{u})-\frac{\mu_{\infty} \Lambda_{e}}{\Lambda_{R}\left(\Lambda_{e}-\Lambda_{R}\right)}\left(\nabla^{2} \dot{\boldsymbol{u}}+\frac{1}{3} \nabla(\nabla \cdot \dot{\boldsymbol{u}})\right) \\
-\rho \ddot{\boldsymbol{u}}+\boldsymbol{b}=\mathbf{0}
\end{array}
$$

where $\mu_{\infty}:=\mu\left(1-\Lambda_{e} / \Lambda_{R}\right)$ is the Lamé constant at $t=\infty$.

It is worth noticing that, differently from the plate model, now viscosity changes the stiffness in a nonproportional way, since the body is considered viscously volumetrically incompressible; for the same reason, damping is of nonproportional type. 


\section{Conclusions}

A linear metamodel of viscoelastic continuum has been formulated. It is expressed in terms of internal variables, having the meaning of the viscous elongations of the (arbitrary in number) dashpots of the underlying rheological model. It was observed that, due to the smallness of damping, the eigenvalue spectrum is well separated, so it entails that the dynamics is rapidly attracted by the space spanned by the (finite-dimensional) central eigenvectors. Two alternative algorithms, based on the center manifold method and the multiple scales method, have been worked out to derive the reduced system. Both methods avoid the evaluation of the central eigenvectors, as instead needed in bifurcation theory. The reduction allows eliminating the (passive) internal variables, while accounting for them as slaves of the (active) observable variables.

Three sample continua of increasing spatial dimensions have been considered for illustrative purposes, namely (a) the transverse vibrations of an Euler-Bernoulli beam resting on a Winkler-type soil, the viscoelastic properties of both being described by the standard three-parameter viscoelastic model, (b) the transverse vibrations of a Kirchhoff plate, obeying to the five-parameter viscoelastic solid model, and (c) a three-dimensional Cauchy continuum, whose volumetric strains are elastic and deviatoric strains viscoelastic. The following conclusions can be drawn.

(1) The lowest-order approximation of the center subspaces supplies a reduced stiffness operator which coincides with the elastic one, but with elastic moduli replaced by the equilibrium values (at infinite time) of the relaxation moduli. This is due to the fact that low damping entails short relaxation times, on the order of the transient duration, so that the steady dynamics occurs when the moduli reach their asymptotic values.

(2) The reduced damping operator is made of a linear combination, with relaxation moduli, of the derivatives which form the stiffness operator. In general, however, damping and stiffness are not proportional, so the Rayleigh simplistic model of damping is not recovered.

(3) The beam on viscoelastic soil can be reduced to an internally and externally damped beam on elastic Winkler soil. Reduction provides the equivalent characteristics. Since viscosity in the two substructures acts independently, the resulting damping is nonproportional. However, if the beam is in air (no soil), damping is of proportional type. A formula has been given for practical design of damping.

(4) The viscoelastic five-parameter plate shows the power of the model, which permits elimination of six internal variables (two for each of the three curvatures), and a strong lowering of dimensions. Due to the fact that the same 
constitutive law has been adopted for all the components of stress, a damping operator proportional to the stiffness has been found.

(5) The three-dimensional Cauchy continuum, in which viscous incompressibility has been adopted, can also be reduced to an internally damped continuum, expressed in terms of displacements and velocities only. The elastic part of the equation consists of the classical Navier equation, in which, however, the Lamé constants are modified as their value at infinite time, in a nonproportional way, due to the different behavior of the deviatoric and spherical parts. Damping is found to be of nonproportional type.

\section{Appendix: A microstructured homogenized one-dimensional continuum}

The chain of oscillators displayed in Figure 4, top, made of $n$ cells of length $h$, is considered. Each cell is a standard three-parameter viscoelastic oscillator, carrying a mass $M$, whose constitutive parameters $c_{0}, c_{v}$, and $b$ are constant along the chain. A homogenization procedure is here developed to obtain an equivalent continuum, representative of the behavior of the chain.

The free dynamics of the continuum is then analyzed under the assumption of small viscous modulus, by making use of asymptotic solutions. It will be shown that a suitable design of the microstructure could lead to spectral properties of the continuum somewhat analogous to those discussed in Section 3.

The considered target continuum is a viscoelastic one-dimensional model, i.e., a bar of length $\ell=n h$ and mass per unit length $m=M / h$ (Figure 4, bottom). In what follows the chain is referred to as the fine model and the homogeneous continuum as the coarse model.

The constitutive law of the periodic structure. The $i$-th cell (Figure 5, left) is taken as the representative volume of the periodic structure. Its constitutive law can

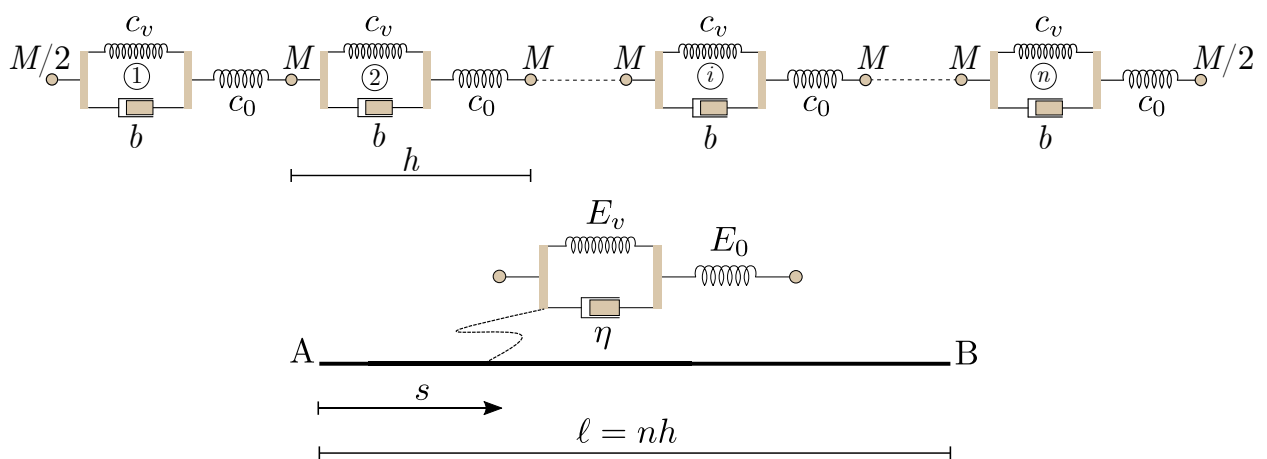

Figure 4. Viscoelastic one-dimensional systems: fine discrete periodic model (top) and homogeneous bar (bottom). 

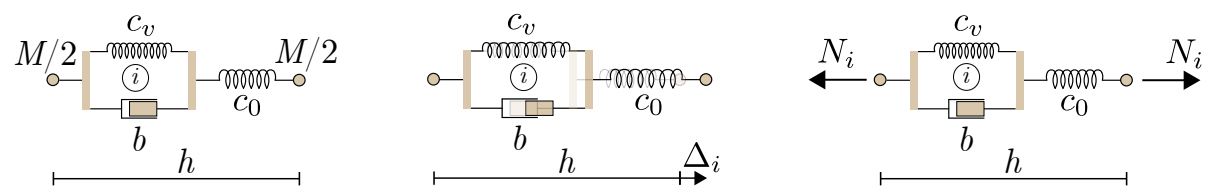

Figure 5. Fine model: cell (left), kinematics (center), and statics (right).

be written by rearranging kinematics (Figure 5, center) and equilibrium (Figure 5, right) equations of the rheological model [Luongo and D'Annibale 2017a]. It reads

$$
\begin{aligned}
N_{i} & =c_{0}\left(\Delta_{i}-\kappa_{i}\right), \\
\dot{\kappa}_{i} & =\frac{c_{0}}{b} \Delta_{i}-\frac{c_{0}+c_{v}}{b} \kappa_{i}
\end{aligned}
$$

where $N_{i}$ is the axial force and $\Delta_{i}$ and $\kappa_{i}$ are the total and viscous displacements, respectively.

The viscoelastic one-dimensional continuum model. The axial motion of a viscoelastic one-dimensional bar is then considered (Figure 4, bottom). Kinematics and equilibrium are described by

$$
\begin{aligned}
u^{\prime} & =\varepsilon, \\
-N^{\prime} & =f-m \ddot{u}
\end{aligned}
$$

where $u(s, t), \varepsilon(s, t), N(s, t)$, and $f(s, t)$ are the axial displacement, the unit extension, the cross-section normal force, and the horizontal external load, respectively, which are functions of the abscissa $s$ and time $t$. Moreover, the prime and the dot denote spatial and time differentiation, respectively. It is worth noticing that (69) are in the same form as (1).

According to the standard three-parameter model, the constitutive law reads

$$
\begin{aligned}
N & =E_{0} A\left(\varepsilon-\varepsilon_{v}\right), \\
\dot{\varepsilon}_{v} & =\frac{E_{0}}{\eta} \varepsilon-\frac{E_{0}+E_{v}}{\eta} \varepsilon_{v}
\end{aligned}
$$

where $E_{0}, E_{v}, \eta$ are material constants, $\varepsilon_{v}$ is an internal variable having the meaning of viscous extensional strain, and $A$ is the area of the cross-section. It is important to remark that (70) are in the same form as (2) of the metamodel.

The constitutive coefficients of the coarse model are determined in the framework of the homogenization procedure. In particular, this is carried out by exploiting the periodicity of the fine model and following the next steps: (i) the constitutive law for one of its cells (68) is written in terms of strains of the coarse model, thus entailing $\Delta_{i}=\varepsilon h$ and $\kappa_{i}=\varepsilon_{v} h$ and (ii) the constitutive law of the cell is attributed 
to a slice of the equivalent bar of the same length. The following identification of the coefficients of the equivalent bar holds:

$$
E_{0} A:=h c_{0}, \quad \frac{E_{0}}{\eta}:=\frac{c_{0}}{b}, \quad \frac{E_{v}}{\eta}:=\frac{c_{v}}{b} .
$$

By combining (69) and (70) in the state space form $(\dot{u}=v)$, the equations of motion of the bar read

$$
\left(\begin{array}{c}
\dot{u} \\
\dot{v} \\
\dot{\varepsilon}_{v}
\end{array}\right)=\left[\begin{array}{ccc}
0 & 1 & 0 \\
\left(E_{0} A / m\right) \partial_{s}^{2} & 0 & -\left(E_{0} A / m\right) \partial_{s}^{1} \\
\left(E_{0} / \eta\right) \partial_{s}^{1} & 0 & -\left(E_{0}+E_{v}\right) / \eta
\end{array}\right]\left(\begin{array}{c}
u \\
v \\
\varepsilon_{v}
\end{array}\right)+\left(\begin{array}{c}
0 \\
f / m \\
0
\end{array}\right) .
$$

These equation are in the form of (3). They must be integrated with initial conditions (assuming the system is initially at rest). Moreover, it is assumed that geometric boundary conditions at the ends prescribe null horizontal displacement, i.e., $u_{\mathrm{A}}=u_{\mathrm{B}}=0$.

Free dynamics. The solution of (72), which (exactly) satisfies the spatial eigenvalue problem, is in the form

$$
\left(\begin{array}{l}
u \\
v
\end{array}\right)=\left(\begin{array}{c}
\hat{u}(t) \\
\hat{v}(t)
\end{array}\right) \sin \left(\frac{k \pi s}{\ell}\right), \quad \varepsilon_{v}=\hat{\varepsilon}_{v}(t) \cos \left(\frac{k \pi s}{\ell}\right)
$$

where $\hat{u}(t), \hat{v}(t)$, and $\hat{\varepsilon}_{v}(t)$ are (time-dependent) amplitudes and $k$ denotes the wave number. Therefore, the associated characteristic equation of the (unforced) system (72) reads

$$
\eta \lambda^{3}+\left(E_{0}+E_{v}\right) \lambda^{2}+\frac{\eta E_{0} A}{m}\left(\frac{k \pi}{\ell}\right)^{2} \lambda+\frac{E_{0} E_{v} A}{m}\left(\frac{k \pi}{\ell}\right)^{2}=0 .
$$

Approximated roots of (74) in the case $\eta$ is small can be determined through a perturbation method (details not reported here). It is found that

$$
\begin{aligned}
\lambda_{1}=-\frac{E_{0}+E_{v}}{\eta}+O(1) & =:-\frac{1}{t_{\mathrm{rel}}}, \\
\lambda_{2,3} & =-\frac{\eta E_{0}^{2} A}{2 m\left(E_{0}+E_{v}\right)^{2}}\left(\frac{k \pi}{\ell}\right)^{2} \pm i \sqrt{\frac{E_{0} E_{v} A}{m\left(E_{0}+E_{v}\right)}\left(\frac{k \pi}{\ell}\right)^{2}}+\mathcal{O}\left(\epsilon^{2}\right)=:-\xi \omega \pm i \omega
\end{aligned}
$$

where $t_{\text {rel }}$ is the relaxation time of the standard three-parameter model. In particular, accordingly to the discussion of Section 3 , it can be seen that one eigenvalue $\lambda_{1}$ is real, of order $\epsilon^{-1}$, and its magnitude is inversely proportional to $t_{\text {rel }}$, while the other two eigenvalues $\lambda_{2}$ and $\lambda_{3}$ are complex-conjugate with order- $\epsilon$ negative real part. Moreover, the following positions hold:

$$
\omega^{2}:=\frac{E_{\infty} A}{m}\left(\frac{k \pi}{\ell}\right)^{2}, \quad \xi:=\frac{\eta E_{0}^{2} A}{2 m \omega\left(E_{0}+E_{v}\right)^{2}}\left(\frac{k \pi}{\ell}\right)^{2},
$$




\begin{tabular}{|ccccc|}
\hline$M[\mathrm{~kg}]$ & $h[\mathrm{~m}]$ & $c_{0}[\mathrm{~N} / \mathrm{m}]$ & $c_{v}[\mathrm{~N} / \mathrm{m}]$ & $b[\mathrm{Ns} / \mathrm{m}]$ \\
1 & 0.2 & 800 & 800 & 50 \\
\hline
\end{tabular}

Table 1. Numerical values of the parameters of the microstructure.

$E_{\infty}:=E_{0} E_{v} /\left(E_{0}+E_{v}\right)$ being the elastic modulus at infinite time. As shown in [Luongo and D'Annibale 2017a], definitions (76) represent the (undamped) frequency and damping ratio of a reduced oscillator, which is able to recover the oscillatory dynamics of the bar after the exponential transient motion decays, and whose motion is governed by the equation

$$
\ddot{u}+2 \xi \omega \dot{u}+\omega^{2} u=\frac{f}{m} .
$$

Discussion about the validity of the perturbation solution. The ratio between the moduli of the real parts of eigenvalues (75) can be written as

$$
r:=\frac{\left|\operatorname{Re} \lambda_{2,3}\right|}{\left|\operatorname{Re} \lambda_{1}\right|}=\frac{E_{0} \omega^{2}}{2 E_{v}} t_{\mathrm{rel}}^{2} .
$$

In particular, by considering $E_{v}$ and $E_{0}$ of the same order of magnitude, e.g., $E_{v}=$ $E_{0} / 2$,

$$
r=\omega^{2} t_{\mathrm{rel}}^{2} \simeq 40\left(\frac{t_{\mathrm{rel}}}{T}\right)^{2}, \quad t_{\mathrm{rel}}=\frac{2}{3} \frac{\eta}{E_{0}},
$$

$T:=2 \pi / \omega$ being the (undamped) period of the structure. By requiring the spectrum of eigenvalues to be well separated, i.e., $r=\mathscr{O}\left(\epsilon^{2}\right)=10^{-2}$, it is found that

$$
\frac{t_{\text {rel }}}{T} \simeq \frac{1}{65} \text {. }
$$

It means that, if the period of the structure is on the order of the second, as occurs in civil structures, $\eta$ must be small, i.e., $\eta \simeq 0.02 E_{0}$. However, the relaxation time of a structural material, as, e.g., identified by a relaxation test, is found of some order of magnitude greater with respect to the structural period. For example for rubber $t_{\text {rel }} \sim 100 \mathrm{~s}$, thus entailing $\eta$ large $\left(\eta \simeq 150 E_{0}\right)$. It is concluded that a well separated spectrum is representative of those viscoelastic structures, in which the relaxation time of the underlying viscoelastic constitutive model is sufficiently smaller than their fundamental period.

The ratio (80) can be easily achieved in the microstructured continuum, by designing its microstructure. As an example, by taking the numerical values reported in Table 1, and $n=20$, the coefficients of Table 2 follow for the equivalent bar. It is found that the first (undamped) period of the structure is $T=2 \mathrm{~s}$, and its relaxation time $t_{\text {rel }}=\frac{1}{32} \mathrm{~s}$, thus corresponding to $t_{\text {rel }} / T=\frac{1}{64}$. Finally, in Figure 6 the very good agreement between the complex-conjugate exact eigenvalues (gray circles) 


\begin{tabular}{|ccccc|}
\hline$m[\mathrm{~kg} / \mathrm{m}]$ & $\ell[\mathrm{m}]$ & $E_{0} A[\mathrm{~N}]$ & $E_{0} / \eta\left[\mathrm{s}^{-1}\right]$ & $E_{v} / \eta\left[\mathrm{s}^{-1}\right]$ \\
5 & 4 & 160 & 16 & 16 \\
\hline
\end{tabular}

Table 2. Numerical values of the parameters of the equivalent bar.

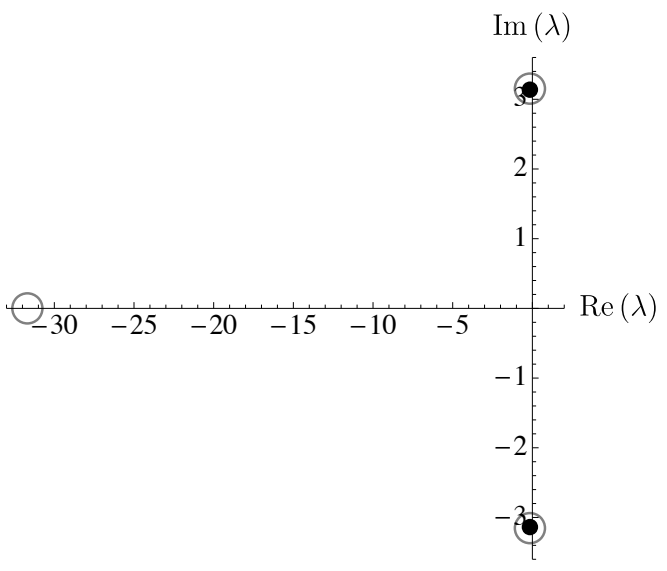

Figure 6. Eigenvalues of the exact (gray circles) and reduced (black dots) systems.

and the asymptotic ones (black dots), obtained by the (unforced) (77), is shown when $k=1$. The exact real eigenvalue is filtered by the reduction procedure.

\section{References}

[Altenbach and Eremeyev 2015] H. Altenbach and V. A. Eremeyev, "On the constitutive equations of viscoelastic micropolar plates and shells of differential type", Math. Mech. Complex Syst. 3:3 (2015), 273-283.

[Barchiesi et al. 2019] E. Barchiesi, M. Spagnuolo, and L. Placidi, "Mechanical metamaterials: a state of the art", Math. Mech. Solids 24:1 (2019), 212-234.

[Baroudi et al. 2019] D. Baroudi, I. Giorgio, A. Battista, E. Turco, and L. A. Igumnov, "Nonlinear dynamics of uniformly loaded Elastica: Experimental and numerical evidence of motion around curled stable equilibrium configurations", Z. Angew. Math. Mech. 99:7 (2019), art. id. e201800121.

[Del Vescovo and Giorgio 2014] D. Del Vescovo and I. Giorgio, "Dynamic problems for metamaterials: review of existing models and ideas for further research", Internat. J. Engrg. Sci. 80 (2014), 153-172.

[Eugster et al. 2019] S. R. Eugster, F. dell'Isola, and D. J. Steigmann, "Continuum theory for mechanical metamaterials with a cubic lattice substructure”, Math. Mech. Complex Syst. 7:1 (2019), 75-98.

[Flügge 1975] W. Flügge, Viscoelasticity, Springer, 1975.

[Frazier and Hussein 2015] M. J. Frazier and M. I. Hussein, "Viscous-to-viscoelastic transition in phononic crystal and metamaterial band structures", J. Acoust. Soc. Am. 138:5 (2015), 3169-3180.

[Fusco and Hale 1989] G. Fusco and J. K. Hale, "Slow-motion manifolds, dormant instability, and singular perturbations", J. Dynam. Differential Equations 1:1 (1989), 75-94. 
[Giorgio et al. 2017] I. Giorgio, U. Andreaus, F. dell'Isola, and T. Lekszycki, "Viscous second gradient porous materials for bones reconstructed with bio-resorbable grafts", Extreme Mech. Lett. 13 (2017), 141-147.

[Golla and Hughes 1985] D. F. Golla and P. C. Hughes, "Dynamics of viscoelastic structures - a time-domain, finite element formulation", J. Appl. Mech. 52:4 (1985), 897-906.

[Guckenheimer and Holmes 1983] J. Guckenheimer and P. Holmes, Nonlinear oscillations, dynamical systems, and bifurcations of vector fields, Applied Mathematical Sciences 42, Springer, 1983.

[Hatada et al. 2000] T. Hatada, T. Kobori, M. Ishida, and N. Niwa, "Dynamic analysis of structures with Maxwell model”, Earthq. Eng. Struct. D. 29:2 (2000), 159-176.

[Lekszycki et al. 1992] T. Lekszycki, N. Olhoff, and J. J. Pedersen, "Modelling and identification of viscoelastic properties of vibrating sandwich beams", Compos. Struct. 22:1 (1992), 15-31.

[Lemaitre and Chaboche 1990] J. Lemaitre and J.-L. Chaboche, Mechanics of solid materials, Cambridge University, 1990.

[Lewandowski et al. 2012] R. Lewandowski, A. Bartkowiak, and H. Maciejewski, "Dynamic analysis of frames with viscoelastic dampers: a comparison of damper models", Struct. Eng. Mech. 41:1 (2012), 113-137.

[Lewińska et al. 2017] M. A. Lewińska, V. G. Kouznetsova, J. A. W. van Dommelen, A. O. Krushynska, and M. G. D. Geers, "The attenuation performance of locally resonant acoustic metamaterials based on generalised viscoelastic modelling”, Int. J. Solids Struct. 126-127 (2017), 163-174.

[Luongo and D'Annibale 2013] A. Luongo and F. D'Annibale, "Double zero bifurcation of nonlinear viscoelastic beams under conservative and non-conservative loads", Int. J. Non-Lin. Mech. 55 (2013), 128-139.

[Luongo and D'Annibale 2017a] A. Luongo and F. D'Annibale, “Invariant subspace reduction for linear dynamic analysis of finite-dimensional viscoelastic structures", Meccanica 52:13 (2017), 3061-3085.

[Luongo and D'Annibale 2017b] A. Luongo and F. D'Annibale, "Nonlinear hysteretic damping effects on the post-critical behaviour of the visco-elastic Beck's beam", Math. Mech. Solids 22:6 (2017), 1347-1365.

[Luongo and Paolone 1999] A. Luongo and A. Paolone, "On the reconstitution problem in the multiple time-scale method", Nonlinear Dynam. 19:2 (1999), 133-156.

[Luongo and Zulli 2012a] A. Luongo and D. Zulli, "Dynamic analysis of externally excited NEScontrolled systems via a mixed multiple scale/harmonic balance algorithm", Nonlinear Dynam. 70:3 (2012), 2049-2061.

[Luongo and Zulli 2012b] A. Luongo and D. Zulli, "A paradigmatic system to study the transition from zero/Hopf to double-zero/Hopf bifurcation”, Nonlinear Dynam. 70:1 (2012), 111-124.

[Luongo and Zulli 2014] A. Luongo and D. Zulli, "Aeroelastic instability analysis of NES-controlled systems via a mixed multiple scale/harmonic balance method", J. Vib. Control 20:13 (2014), 19851988.

[Luongo et al. 2016] A. Luongo, F. D'Annibale, and M. Ferretti, "Hard loss of stability of Ziegler's column with nonlinear damping", Meccanica 51:11 (2016), 2647-2663.

[Manimala and Sun 2014] J. M. Manimala and C. T. Sun, "Microstructural design studies for locally dissipative acoustic metamaterials", J. Appl. Phys. 115:2 (2014), art. id. 023518.

[Maugin and Muschik 1990] G. A. Maugin and W. Muschik, "Thermodynamics with internal variables, I: General concepts”, J. Non-Equil. Thermod. 19:3 (1990), 217-249. 
[McTavish and Hughes 1993] D. J. McTavish and P. C. Hughes, "Modeling of linear viscoelastic space structures", J. Vib. Acoust. 115:1 (1993), 103-110.

[Moreau 1970] J. J. Moreau, "Sur les lois de frottement, de plasticité et de viscosité", C. R. Acad. Sci. Paris A 271 (1970), 608-611.

[Muscolino and Palmeri 2007] G. Muscolino and A. Palmeri, "Response of beams resting on viscoelastically damped foundation to moving oscillators", Int. J. Solids Struct. 44:5 (2007), 13171336.

[Narayanan and Beskos 1982] G. V. Narayanan and D. E. Beskos, "Numerical operational methods for time-dependent linear problems", Internat. J. Numer. Methods Engrg. 18:12 (1982), 1829-1854.

[Nayfeh 2000] A. H. Nayfeh, Perturbation methods, Wiley, New York, 2000.

[Nayfeh and Mook 1979] A. H. Nayfeh and D. T. Mook, Nonlinear oscillations, Wiley, New York, 1979.

[Schanz 1999] M. Schanz, "A boundary element formulation in time domain for viscoelastic solids", Comm. Numer. Methods Engrg. 15:11 (1999), 799-809.

[Schanz 2001] M. Schanz, Wave propagation in viscoelastic and poroelastic continua: a boundary element approach, Lecture Notes in Applied Mechanics 2, Springer, 2001.

[Shaw and Pierre 1991] S. W. Shaw and C. Pierre, "Nonlinear normal modes and invariant manifolds", J. Sound Vib. 150:1 (1991), 170-173.

[Sim and Kwak 1988] W. J. Sim and B. M. Kwak, "Linear viscoelastic analysis in time domain by boundary element method”, Comput. Struct. 29:4 (1988), 531-539.

[Syngellakis 2003] S. Syngellakis, "Boundary element methods for polymer analysis", Eng. Anal. Bound. Elem. 27:2 (2003), 125-135.

[Troger and Steindl 1991] H. Troger and A. Steindl, Nonlinear stability and bifurcation theory: an introduction for engineers and applied scientists, Springer, 1991.

[Wiggins 2003] S. Wiggins, Introduction to applied nonlinear dynamical systems and chaos, 2nd ed., Texts in Applied Mathematics 2, Springer, 2003.

Received 21 Oct 2019. Revised 9 Jan 2020. Accepted 18 Feb 2020.

ANGELO LUONGO: angelo.luongo@univaq.it

Dipartimento di Ingegneria Civile, Edile-Architettura e Ambientale, Il Centro Internazionale di Ricerca per la Matematica e Meccanica dei Sistemi Complessi, Università degli Studi dell'Aquila, L'Aquila, Italy

FRANCESCO D'ANNIBALE: francesco.dannibale@univaq.it

Dipartimento di Ingegneria Civile, Edile-Architettura e Ambientale, Il Centro Internazionale di Ricerca per la Matematica e Meccanica dei Sistemi Complessi, Università degli Studi dell'Aquila, L'Aquila, Italy 



\title{
THE METHOD OF VIRTUAL POWER IN THE MECHANICS OF CONTINUOUS MEDIA I: SECOND-GRADIENT THEORY
}

\author{
PAUl Germain
}

Translated by Marcelo Epstein and Ronald E. Smelser

The systematic application of the definition of internal forces, by means of the virtual power produced in a class of virtual motions, leads to a consistent mathematical representation of stresses and strains in any given mechanical model. It is thus possible to write the statical and dynamical equations and to state well posed boundary value problems. The second-gradient theory, presented here by way of example, can be developed without any ambiguity. An essential distinction is drawn between intrinsic and classical stresses so as to avoid certain issues of interpretation. It is shown that all the results of classical linear elasticity can be immediately extended to the case of second-gradient elastic media. The constitutive equations of nonlinear elasticity are also formulated.

\section{Main notation}

\section{(1) Kinematic quantities}

velocity

strain-rate tensor

rotation-rate tensor

rotation-rate vector

tangential component

rotation-gradient tensor

symmetric part of the tensor of

second gradient of the velocities
$U_{i}(\underline{x}, t)$

$D_{i j}=\frac{1}{2}\left(U_{i, j}+U_{j, i}\right)$

$\Omega_{i j}=\frac{1}{2}\left(U_{i, j}-U_{j, i}\right)$

$\omega_{i}=-\frac{1}{2} \varepsilon_{i p q} \Omega_{p q}, \Omega_{i j}=-\varepsilon_{i j k} \omega_{k}$

$\widetilde{\omega}_{i}$

$K_{i j}=\omega_{i, j}=-\frac{1}{2} \varepsilon_{i p q} \Omega_{p q, j}=-\frac{1}{2} \varepsilon_{i p q} U_{p, q j}$

$K_{i j k}=\frac{1}{3}\left(U_{i, j k}+U_{j, k i}+U_{k, i j}\right)$

\section{Communicated by .}

Original French: "La méthode des puissances virtuelles en mécanique des milieux continus, première partie: Théorie du second gradient", Journal de Mécanique 12:2 (1973), 235-274. Used with permission. Translators' footnotes are identified with the symbols TN.

See also the next article in this issue: Marcelo Epstein and Ronald E. Smelser, "An appreciation and analysis of Paul Germain's 'The method of virtual power in the mechanics of continuous media, I: Second-gradient theory'”, Math. Mech. Complex Systems, 8:1 (2020), pp. 191-199. 
(2) Internal and external forces

volumetric force

volumetric couple

volumetric symmetric double force

surface traction (stress vector)

surface double traction (couple stress vector)

doubly normal stress (surface density)

$f_{i}$

edge stress (line density)

$C_{i j}\left(=-C_{j i}\right)$

$\Phi_{i j}\left(=\Phi_{j i}\right)$

$T_{i}$

$\tilde{M}_{i}$

surface force on $\partial S$

tangential surface couple

$N$

$R_{i}$

$t_{i}$

$\tilde{m}_{i}$

doubly normal force

$n$

edge force (line density)

$r_{i}$

intrinsic stress tensor (1st order)

$\sigma_{i j}\left(=\sigma_{j i}\right)$

intrinsic stress tensors (2nd order)

$\begin{cases}\mu_{i j} & \left(\mu_{i i}=0\right) \\ \mu_{i j k} & \text { (completely symmetric) }\end{cases}$

(3) Derivative operators on a surface (with unit vector $n_{i}$ )

normal gradient

scalar function $\varphi$

$D \varphi=n_{l} \varphi_{, l}$

vector function $V_{i}$

$D V_{i}=n_{l} V_{i, l}$

tangential gradient

scalar function $\varphi$

$D_{i} \varphi=\varphi_{, i}-n_{i} D \varphi$

vector function $V_{i}$

$D_{j} V_{i}=V_{i, j}-n_{j} D V_{i}$

(4) Small strains

displacement

strain tensor

rotation tensor

rotation vector

tangential component

rotation-gradient tensor

symmetric part of the tensor of

second gradient of the displacements

$$
\begin{aligned}
& X_{i}(\underline{x}, t) \\
& \varepsilon_{i j}=\frac{1}{2}\left(X_{i, j}+X_{j, i}\right) \\
& \varphi_{i j}=\frac{1}{2}\left(X_{i, j}-X_{j, i}\right) \\
& \varphi_{i}=-\frac{1}{2} \varepsilon_{i p q} \varphi_{p q} \\
& \widetilde{\varphi}_{i} \\
& \eta_{i j}=-\frac{1}{2} \varepsilon_{i p q} X_{p, q j} \\
& \eta_{i j k}=\frac{1}{3}\left(X_{i, j k}+X_{j, k i}+X_{k, i j}\right)
\end{aligned}
$$

(5) Finite strains

gradient matrix

Green-Lagrange strain tensor

intrinsic Piola-Kirchhoff stress tensor (1st order)

intrinsic stress tensors (2nd order)

$F_{i \alpha}=\frac{\partial x_{i}}{\partial a_{\alpha}}$

$L_{\alpha \beta}=\frac{1}{2}\left(F_{i \alpha} F_{i \beta}-\delta_{\alpha \beta}\right)$

$s_{\alpha \beta}$

$\Pi_{\alpha \beta} \quad\left(\Pi_{\alpha \alpha}=0\right)$

$\left\{\Pi_{\alpha \beta \gamma}\right.$ (completely symmetric) 


\section{Introduction}

It has long been recognized that in mechanics there are two ways to represent mathematically the forces exerted at a given time $t$ upon a system $S$. The first, age-old, way consists in representing a force by means of a vector, a mathematical entity that has an origin, a direction, and a magnitude. The completely natural generalization of this idea when attempting to represent the forces exerted on a continuous system leads to a description in terms of a field of vectors associated with a measure, and it is in this way that one speaks of "volumetric forces", "surface forces", "forces per unit mass", etc. While forces are thus represented, it is desirable to utilize as a basic statement of dynamics the fundamental law stipulating that "there exists at least one reference (frame and time), said to be absolute, in which at each time and for each system, the wrench produced by the masses times the accelerations is equal to the wrench of the exterior forces exerted on the system".

But there exists also, at least since d'Alembert, a second possible avenue, namely, the method of virtual power (or virtual work). Contrary to what is sometimes believed, this second way is as absolutely natural as the first, since it is nothing but the expression of a very common physical experience. If one wants to know if a suitcase is heavy, one tries to lift it slightly; to estimate the tension in a transmission belt, one displaces it a little bit from its stable position; and it is while trying to push a car that one becomes aware of the presence of the internal and external friction forces opposing the motion. From the mathematical point of view, the situation can be described as follows: at a given time $t$, one considers on $S$ a vector field $\boldsymbol{V}$ that defines at that instant a virtual movement of $S$ - the vectors of the field representing the velocities or the elementary (infinitesimal) displacements during an elementary (infinitesimal) time $\delta t-$; the forces that produce this virtual movement $\boldsymbol{V}$ are known if their "virtual power" $\mathscr{P}$ (a real number associated with $\boldsymbol{V}$ ) is known. More precisely, we consider a set $\mathscr{V}$ of virtual motions $\boldsymbol{V}$, where $\mathscr{V}$ is a normed vector space, and we say that we know the forces exerted on $S$ by the space $\mathscr{V}$ if there exists a continuous linear form $\mathscr{L}(\boldsymbol{V})$, defined on $\mathscr{V}$, whose value for each field $\boldsymbol{V}$ is equal to the virtual power of these forces during the virtual motion defined by $\boldsymbol{V}$.

The essential idea of this second avenue is that of "duality". Moreover, this avenue is not only very close to everyday experience, as we have already remarked, but it is also very versatile; according to the choice of a vector space more, or less, "vast", we will have a description of forces more, or less, fine. Thus, we can consider only those expressions that describe the forces that we need by conveniently choosing the space $\mathscr{V}$. Once $\mathscr{V}$ has been fixed, the set of forces recognized by $\mathscr{V}$ form themselves a vector space, namely, the dual $\mathscr{V}^{*}$ of $\mathscr{V}$.

We must acknowledge that the notion of a linear map over a vector space is more abstract than that of a vector field, and it is for this reason that the "virtual 
power approach" has always - at least up until now - appeared to be more difficult. Above all, one has to acknowledge that at the time when the notion of virtual motion was introduced in mechanics the mathematical idea of duality had not yet been sufficiently elaborated so as to completely translate this new notion, which in a certain sense can be considered as the precursor of the notions of measure or of distribution. It is only when the space $\mathscr{V}$ is of finite dimension that no special difficulty arises, and this is why, very early on, we have witnessed the development of the analytical mechanics of systems of a finite number of degrees of freedom, which utilizes in fact, with the notion of "generalized forces", a description of forces by means of the concept of virtual power.

The situation today is different. Functional analysis has been considerably developed and its applications to mechanics, and most particularly to the mechanics of continuous media, are already numerous and of great importance, as it is demonstrated, for example, by the recent work of Duvaut and Lions [1]. The concept of duality is imparted very early on in university curricula. Moreover, the time seems to have arrived to attempt a rather systematic application of the notion of virtual power to continuum mechanics. Such is the objective of this article and of those that will follow under the same general heading.

When utilizing a description of forces by means of virtual power, the most suitable fundamental statement of the laws of dynamics is the principle of virtual power. We will limit ourselves in this first article to the case of statics, a case where this principle can be stated as follows:

\section{"In an absolute reference, at each time $t$ and for every system, the virtual power of all the forces, internal as well as external, applied to the system vanishes, whatever the virtual motion considered."}

As is well known, the statement valid for the dynamic case is obtained by adding to the external forces the absolute forces of inertia.

Our procedure is thus very simple and elementary. We want to show that a mechanical theory - and in particular a theory of the mechanics of continuous media - is completely determined once we provide the space $\mathcal{V}$ of virtual motions that we intend to consider and that establishes in some sense the degree of fineness of the theory. The corresponding representation of the forces is deduced by duality, and the collection of all the equations of statics (and more generally, of dynamics) is obtained by application of the principle of virtual power. By way of example, we will start in Section 2 with a short review of the cases of a material point and of a rigid body, classical cases where $\mathscr{V}$ is of finite dimension. After some general remarks about the application of the method to continuous media (Section 3), we will satisfy ourselves in Section 4 with an examination of the so-called first-gradient 
theory - which is in fact nothing but a slight generalization of the classical theory - and in Section 5 we will present the second-gradient theory. To avoid any error of interpretation, let us note at the outset that our terminology differs from that employed by other authors; the second gradient under consideration here is that of the field of velocities, so that the theory thus named must be compared with the theory of first gradient of the strain (for instance, that developed by Mindlin and Eshel [2]). The results obtained lay the groundwork for the formulation of the thermodynamic properties of the media considered and, consequently, the formulation of the constitutive laws, at least if we resort to the method of local state (see, for example, Germain [3]). By way of illustration we indicate in Section 6 the general features of linear elasticity within the second-gradient theory, and in Section 7, some remarks about nonlinear elasticity that lead in a simple way to the formulation of constitutive laws.

It is not our intention in this article to provide many new results; a large part of the results established below can be found in the literature, in a more or less equivalent form, particularly in the articles listed in the bibliography, at least for the case of elastic media. But, to the best of this author's knowledge, the use of the method of virtual power to define a mechanical model within a given framework of representation has not been the object of a systematic exposition. The second-gradient theory, offered here by way of illustration, permits us to reveal the advantages of this approach. On the one hand, the results remain valid if one wants to take into consideration nonelastic effects. On the other hand, a certain number of difficulties of interpretation that are often present in previous presentations are here automatically removed.

We will not insist here on the strictly mechanical interest of the second-gradient theory, and we refer the reader in this regard to the articles of Mindlin and Eshel [2] and of Toupin [6] and, above all, to the contributions of Casal [13; 14; 15], who has clearly exposed the points of contact between this theory and that of the phenomena of capillarity, thus bringing to light a very interesting physical interpretation, which has not yet received the attention that it deserves.

\section{Elementary remarks on the material point and the rigid body}

The case of the material point is reviewed here only for reference: at a given time, a virtual motion of the point $M$ is determined by giving the virtual velocity $\boldsymbol{V}_{M}$ of $M$; the space $\mathscr{V}$ is, therefore, a Euclidean vector space (of dimension 3). A linear form on $\mathscr{V}$ can be written as an inner product; thus, it determines an element $\boldsymbol{F}_{M}$ of the dual space, and one can write the virtual power as

$$
\mathscr{P}=\mathscr{L}\left(\boldsymbol{V}_{M}\right)=\boldsymbol{F}_{M} \cdot \boldsymbol{V}_{M} .
$$


In this way, we recover the representation of the forces exerted on a point by the force vector $\boldsymbol{F}_{M}$, such as provided by the first kind of description of forces recalled at the beginning of the introduction.

Analogously, the case of the rigid body leads to a classical result, although its meaning does not always emerge quite as clearly. Let us consider at a fixed time $t$ a system $S$, which we will refer to an orthonormal frame - with $x_{1}, x_{2}, x_{3}$ indicating the coordinates of a point of $S$, which we will simply denote by $\underline{x}$. It is known that, if $S$ is a rigid body, the velocity field $U_{i}(\underline{x})$ of the points of $S$ satisfies an identity of the form

$$
U_{i}(\underline{x})=U_{i}(\underline{o})+\Omega_{i j} x_{j},
$$

where $\Omega_{i j}$ is a skew-symmetric matrix, independent of $\underline{x}$, called the rotation-rate matrix, representing, in this frame, the skew-symmetric second-order rotation-rate tensor (or spin tensor). A field that satisfies the identity (1) for every $\underline{x}$ in $S$ is said to be defined by means of a twist (or a distributor). A twist is thus defined by the six scalars $\Omega_{i j}=-\Omega_{j i}$ and $U_{i}(\underline{o})$, which are called its elements of reduction at the origin. It is also equally well defined by its elements of reduction at any other point of $S$.

At some fixed time $t$, let us take as the space of virtual motions $\mathscr{V}$ the (6-dimensional) vector space $\mathscr{C}$ of the twists, a twist being denoted by $\{\mathscr{C}\}$. We say that these virtual motions "preserve the rigidity of $S$ " if $S$ is a rigid body or, if $S$ is a deformable medium, that these are "virtual motions that rigidify $S$ ". The virtual power of the forces applied on $S$ is a linear form over $\mathscr{C}$, namely,

$$
\mathscr{P}=\mathscr{L}(\{\mathscr{C}\}) .
$$

We say that this form defines the screw or wrench of the forces, which we denote by $[\mathcal{T}]$. Such a screw is an element of the dual space $\mathscr{T}$ of the space $\mathscr{C}$. If we represent $\{\mathscr{C}\}$ by its elements of reduction at the origin, we can write $\mathscr{P}$ in the form

$$
\mathscr{P}=T_{i}(\underline{o}) U_{i}(\underline{o})+M_{i j}(\underline{o}) \Omega_{i j}
$$

The real numbers $T_{i}(\underline{o})$ and $M_{i j}(\underline{o})$ are the elements of reduction of [F] at the origin. It is clear that, since $\Omega_{i j}$ is a skew-symmetric matrix, we can assume without loss of generality that $M_{i j}$ is also skew-symmetric. It is also clear that, since $U_{i}$ and $\Omega_{i j}$ are, respectively, components of a vector and of a second-order skew-symmetric tensor, the same is true for $T_{i}$ and $M_{i j}$, respectively.* Naturally, we could also have expressed the linear form (2) while representing $\{\mathscr{b}\}$ by its elements of reduction at another point $\underline{x}$ arbitrarily chosen, and we could have written

$$
\mathscr{P}=T_{i}(\underline{x}) U_{i}(\underline{x})+M_{i j}(\underline{x}) \Omega_{i j} .
$$

\footnotetext{
*In other words, these are also components of a vector and of a tensor, respectively. — (TN)
} 
Subtracting (3) from (4), and invoking (1), we obtain

$$
\left(T_{i}(x)-T_{i}(\underline{o})\right) U_{i}(\underline{o})+\left(M_{i j}(\underline{x})-M_{i j}(\underline{o})+T_{i}(\underline{x}) x_{j}\right) \Omega_{i j}=0 .
$$

This equation holds true for arbitrary values of $U_{i}(\underline{o})$ and of $\Omega_{i j}=-\Omega_{j i}$. This implies that the coefficient of $U_{i}(\underline{o})$ must vanish identically and that the coefficient of $\Omega_{i j}$ must be symmetric in $i$ and $j$. The vector $\boldsymbol{T}$, called the vector or resultant of the wrench [ $\mathscr{T}]$, is therefore independent of $\underline{x}$, and the skew-symmetric secondorder tensor field defined by the matrices $M_{i j}$ is an affine linear function of the coordinates and satisfies the identity

$$
M_{i j}(\underline{x})=M_{i j}(\underline{o})+x_{[i} T_{j]},
$$

where $A_{[i j]}$ denotes the skew-symmetric part of $A_{i j}{ }^{*}$

The preceding treatment is valid regardless of the (finite) dimension of the Euclidean space in which the system $S$ is found. The velocity field (1) and the moment field (5), associated, respectively, to the twist and the wrench, are entities of different mathematical nature. Indeed, the velocity is a vector field while the moment is a second-order skew-symmetric tensor field. It is only in the case of a 3-dimensional space that certain correspondences between these two entities can be made. Let us introduce in this space the alternating tensor with components $\varepsilon_{i j k}$, and let us define

$$
\omega_{k}=-\frac{1}{2} \varepsilon_{k i j} \Omega_{i j}, \quad m_{k}=-\varepsilon_{k i j} M_{i j} .
$$

Equations (1) and (5) can then be rewritten in the classical form

$$
\boldsymbol{U}_{M}=\boldsymbol{U}_{0}+\omega \wedge \boldsymbol{O M} ; \quad \boldsymbol{m}_{M}=\boldsymbol{m}_{0}+\boldsymbol{M O} \wedge \boldsymbol{T}
$$

while $\mathscr{P}$ can be expressed as

$$
\mathscr{P}=\boldsymbol{T} \cdot \boldsymbol{U}_{M}+\boldsymbol{\omega} \cdot \boldsymbol{m}_{M}=[\mathscr{T}] \cdot\{\mathscr{C}\}
$$

The vector $\omega$ is the rate of rotation (or angular velocity) vector of the twist $\{\mathscr{b}\}$. The vector field $\boldsymbol{m}_{M}$ is the moment field of the wrench [T].

Let us underscore once again the significance of the results just obtained: in the mechanics of rigid bodies, it is futile or superfluous to represent the forces acting on the rigid body other than by means of the wrench that they determine; any other finer representation is redundant.

*Note, as a matter of detail, that on taking the skew-symmetric part of the tensor product $\boldsymbol{x} \otimes \boldsymbol{T}$, a factor of $\frac{1}{2}$ is introduced, thus explaining the lack of it later in the second equation (6). - (TN) 


\section{General remarks on the application of the virtual power method in continuum mechanics}

From the outset, let us note the essential role played by the following axiom of the virtual power of the internal forces:

The virtual power of the internal forces of a system $S$ vanishes for any rigidifying virtual motion of $S$ at the time $t$ being considered.

Let us recall that it is thanks to this axiom that the statement of the principle of virtual power entails the fundamental classical law of mechanics. If, at an arbitrary time $t$, we consider a rigidifying virtual motion of $S$, defined by a twist $\{\mathscr{C}\}$, the virtual power of all the applied forces is reduced to that of the external forces alone, and since $S$ is assumed to be in equilibrium, the virtual power, written as $[\mathscr{T}] \cdot\{\mathscr{C}\}$, where $[\mathscr{T}]$ is the wrench of the external forces, must vanish for arbitrary $\{\mathscr{C}\}$. We immediately deduce that $[\mathscr{T}]=\mathbf{0}$, which is precisely the statement of the fundamental law of statics.

The remarks that follow do not have the compulsory and general character of the axiom just formulated; rather, they constitute working hypotheses that could be called into question in theories other than those presented below by way of illustration of the general method.

(a) The systems $S$ to be considered will always be 3-dimensional. We will assume that $S$ is a connected and bounded open domain of the Euclidean space and that its boundary $\partial S$ is piecewise twice continuously differentiable, namely that, except on certain lines which are the edges of $\partial S$, the surface $\partial S$ has at each of its points a well defined exterior unit normal vector, say $\boldsymbol{n}$, and a curvature tensor which is continuous in a neighborhood of each $P$ belonging to $\partial S$.

(b) We will apply the principle of virtual power, be it to $S$ or to any subsystem $\mathscr{D}$ of $S$, for which we will make the same regularity assumptions as for $S$.

(c) The functions chosen to describe the virtual motion of $\mathscr{D}$, that is, those functions that define an arbitrary element of the normed vector space $\mathscr{V}$, will be assumed to be continuously differentiable over the closure $\mathscr{D}+\partial \mathscr{D}$ of $\mathscr{D}$, as many times as necessary (for example, infinitely differentiable).

(d) The natural language suited to such a theory is that of the theory of distributions. Nevertheless, in order to simplify the exposition and so as to recover directly the classical formulas, we will not make use of it here. This is tantamount to admitting that the distributions that represent the forces are sufficiently regular to be defined in terms of densities, that is, (continuously differentiable) functions defined over certain manifolds. This simplification is more often than not a legitimate one, since we are dealing with notions 
pertaining to continuous media, themselves a depiction of an essentially discontinuous reality. The linear forms that will define the virtual power can, therefore, be written by means of volume, surface, or line integrals.

In fact, the results obtained under this working hypothesis remain valid in the general case as long as we interpret the various quantities appearing there in the sense of distributions.

(e) The virtual power of the internal forces in the subsystem $\mathscr{D}$ will be denoted as $\mathscr{P}_{(i)}(\mathscr{D})$. We will always assume that it can be expressed in the form of a volume integral over the open set $\mathscr{D}$.

(f) The external forces exerted on the subsystem $\mathscr{D}$, interior to $S$, will be assumed to be of two types. The first consists of these forces exerted on $\mathscr{D}$ by the systems external to $S$; these are the actions at a distance, which, moreover, will be considered in general as given. We will denote their virtual power by $\mathscr{P}_{(d)}$, and we will assume that it is expressed in the form of a volume integral over the open set $\mathscr{D}$. The second kind of external forces consists of those forces exerted on $\mathscr{D}$ by the parts of $S$ exterior to $\mathscr{D}$. We will assume here that, as is customary in the mechanics of continuous media, these are contact forces - thus implying that the actions at a distance originating within $S$ are negligible. The virtual power of the contact forces will be denoted by $\mathscr{P}_{(c)}$, which will be expressible by means of a surface integral ${ }^{1}$ over $\partial \mathscr{D}$.

Analogously, we will assume that the external forces exerted on $S$ also comprise actions at a distance and contact forces on $\partial S$.

(g) Since we limit ourselves to the case of statics, the principle of virtual power is expressed by the equation

$$
\mathscr{P}_{(d)}+\mathscr{P}_{(c)}+\mathscr{P}_{(i)}=0
$$

which must be satisfied for any subdomain $\mathscr{D}$ and any virtual motion considered in $\mathscr{V}$. The relations that express the necessary and sufficient conditions for this to be true constitute the set of equations of statics for the system under consideration.

The meaning of these remarks will become clearer through the two examples that we will presently consider.

\section{First-gradient theory}

The first-gradient theory is, in fact, a rather simple generalization of the classical formulation of the theory of continuous media. The name given to this first-gradient

${ }^{1}$ As we will see in Section 5, in certain cases it may be appropriate to include an additional term expressed in the form of a line integral (over the edges of $\partial \mathscr{D})$. 
theory arises from the fact that for a given subsystem $\mathscr{D}$ the space of virtual motions $\mathscr{V}$ is that of continuous and at least once continuously differentiable velocities over the closure $\mathscr{D}+\partial \mathscr{D}$ of $\mathscr{D}$, where the norm of $\mathscr{V}$ is that of the uniform convergence for the velocities and their first derivatives with respect to the coordinates $x_{i}$. We will denote by $U_{i}$ the velocity components and by $U_{i, j}$ their first derivatives. We will, moreover, introduce the canonical decomposition of the velocity gradient into a symmetric part and a skew-symmetric part, namely,

$$
U_{i, j}=D_{i j}+\Omega_{i j} ; \quad D_{i j}=D_{j i}, \quad \Omega_{i j}=-\Omega_{j i} .
$$

We know that $D_{i j}$ is the matrix that represents the strain-rate tensor and that $\Omega_{i j}$ is the matrix representing the rotation-rate tensor. The continuous linear forms on this space $\mathscr{V}$ are, in all generality, distributions of order 1 , that is, measure derivatives. But we have already explained in the preceding section that we will not resort here to this generality and that we will assume that the virtual power can be expressed by means of integrals.

4.1. We shall always commence by formulating the virtual power of the internal forces. This will be done for two reasons. In the first place, this is the essentially new notion brought about by continuum mechanics. In the second place, we have at our disposal the axiom stated above, which permits us to simplify its expression. Moreover, we will find that, in writing the virtual work of the external forces, we will be guided by the results already gained for the expression of the virtual power of the internal forces.

We know that $\mathscr{P}_{(i)}$ is a volume integral over $\mathscr{D}$ (in accordance with remark (e) above). We will write, therefore,

$$
\mathscr{P}_{(i)}=-\int_{\mathscr{D}} p d v
$$

Except for the sign, $p$ is the virtual power of the internal forces per unit volume, or the energy of the internal forces per unit volume. Furthermore, by hypothesis, $p$ must be a linear form in the arguments $U_{i}, D_{i j}$, and $\Omega_{i j}$. But, by virtue of the axiom, we can state:

Proposition 1. The density $p$ can be written in the form

$$
p=\sigma_{i j} D_{i j}
$$

where $\sigma_{i j}$ is a symmetric matrix representing a symmetric second-order tensor called the intrinsic stress tensor (of order 1), which is an objective quantity.

The proof is straightforward. It is clear that, without loss of generality, we can assume that $\sigma_{i j}$ is symmetric with respect to the indices $i$ and $j .{ }^{*}$ Since $D_{i j}$

*Assuming, of course, that we're in the case where (12) is valid. - (TN) 
represents a tensor, the same must be true for $\sigma_{i j}$, as can be concluded by a change of frame at the time under consideration. Moreover, by virtue of the axiom, $\mathscr{P}_{(i)}$ and $p$ preserve their values under a change of reference, since evidently the difference of the velocity fields of one and the same virtual motion as observed in two different references is the velocity field of some twist. We still need to show that $p$ can depend neither on $U_{i}$ nor on $\Omega_{i j}$. If, for instance, there were in (12) a term in $U_{i}$ having a coefficient not identically zero in the neighborhood of a point $M$ of $S$, one could find a subsystem $\Delta$ of $S$, containing $M$, and a virtual motion of translation defined on $\Delta$, for which $p$ would not vanish identically, contrary to the stipulation of the axiom. The impossibility of having a nonvanishing term in $\Omega_{i j}$ in (12) can be established by a similar reasoning. The proposition is thus proven.

We assume (remark (d)) that the components $\sigma_{i j}$ are continuously differentiable in $x_{i}$. The divergence theorem permits us to derive, having duly noted that $\sigma_{i j} D_{i j}=$ $\sigma_{i j} U_{i, j}$, the following useful expression for the virtual power of the internal forces:

$$
\mathscr{P}_{(i)}=\int_{\mathscr{D}} \sigma_{i j, j} U_{i} d v-\int_{\partial \mathscr{D}} \sigma_{i j} n_{j} U_{i} d a .
$$

If we take $U_{i}$ as the velocity field of a twist, ${ }^{*}$ the left-hand side of (13) vanishes, ${ }^{\dagger}$ so that, incidentally, we obtain the following:

Proposition 2. The wrench defined by the volumetric density $\sigma_{i j, j}$ in $\mathscr{D}$ is equal to that defined by the surface density $\sigma_{i j} n_{j}$ on $\partial \mathscr{D}$.

This result is usually conveyed in more compact notation ${ }^{\ddagger}$ as

$$
\left[\sigma_{i j, j}\right]_{\mathscr{D}}=\left[\sigma_{i j} n_{j}\right]_{\partial \mathscr{D}} .
$$

4.2. We will presently formulate the power of the external forces exerted on $\mathscr{D}$, while adhering to the working hypotheses stated in the preceding section. We will proceed systematically by writing general linear forms over $\mathscr{V}$ and postponing until the end of this section a brief discussion of the physical meaning of the quantities used. As far as $\mathscr{P}_{(d)}$ is concerned, we will write

$$
\mathscr{P}_{(d)}=\int_{\mathscr{D}}\left(f_{i} U_{i}+C_{i j} \Omega_{i j}+\Phi_{i j} D_{i j}\right) d v .
$$

This definition implies that the external actions at a distance can be represented by

- a field of volumetric forces defined by the density $f_{i}$,

- a field of volumetric couples defined by the density $C_{i j}$, representing a skewsymmetric tensor, namely, $C_{i j}=-C_{j i}$,

${ }^{*}$ That is, a rigidifying motion. - $(\mathrm{TN})$

${ }^{\dagger}$ According to the axiom. - (TN)

¥That is, using screw (or torsor) "notation" as in Equation (8) of [16]. — (TN) 
- a field of volumetric "symmetric double forces" defined by the density $\Phi_{i j}$, representing a symmetric tensor, namely, $\Phi_{i j}=\Phi_{j i}$.

It is convenient to transform the equality (15) following the procedure used above. Noting the identities

$$
\begin{aligned}
& C_{i j} \Omega_{i j}=C_{i j} U_{i, j}=\left(C_{i j} U_{i}\right)_{, j}-C_{i j, j} U_{i}, \\
& \Phi_{i j} D_{i j}=\Phi_{i j} U_{i, j}=\left(\Phi_{i j} U_{i}\right)_{, j}-\Phi_{i j, j} U_{i},
\end{aligned}
$$

and applying the divergence theorem, we obtain

$$
\mathscr{P}_{(d)}=\int_{\mathscr{D}}\left(f_{i}-C_{i j, j}-\Phi_{i j, j}\right) U_{i} d v+\int_{\partial \mathscr{D}}\left(C_{i j}+\Phi_{i j}\right) n_{j} U_{i} d a .
$$

All that remains is to deal with the external contact forces. Their virtual power is defined by a scalar surface density which is, a priori, a linear function of $U_{i}$ and of the first derivatives of $U_{i}$. But, anticipating the formulation of the principle of virtual power, we become aware that these last terms vanish identically, since they could not be possibly balanced by any analogous term in the expressions (13) and (16) of the virtual power of the internal forces and the actions at a distance. Moreover, we can obtain this result in an absolutely explicit fashion from the expression (34), given farther below. We will, therefore, simply write

$$
\mathscr{P}_{(c)}=\int_{\partial \mathscr{D}} T_{i} U_{i} d a,
$$

where, by definition, $T_{i}$ represents the stress vector at a point of $\partial \mathscr{D}$ acting perpendicularly to $\partial \mathscr{D}$; this is a surface density of contact forces.

4.3. It remains to apply the principle of virtual power, that is, (9). Taking (13), (16), and (17) into consideration, we obtain

$$
0=\int_{\mathscr{D}}\left(f_{i}+\sigma_{i j, j}-C_{i j, j}-\Phi_{i j, j}\right) U_{i} d v+\int_{\partial \mathscr{D}}\left(T_{i}-\left(\sigma_{i j}-C_{i j}-\Phi_{i j}\right) n_{j}\right) U_{i} d a .
$$

We are thus led to define

$$
\tau_{i j}=\sigma_{i j}-C_{i j}-\Phi_{i j} .
$$

By definition, $\tau_{i j}$ represents the stress tensor.

Let us apply first the identity (18) taking as $U_{i}$ an arbitrary field that vanishes outside a compact set contained in $\mathscr{D}$. In that case, we are just left with the volume integral, and since $U_{i}$ is otherwise arbitrary, we obtain at each point of this compact set, that is, at each interior point of $\mathscr{D}$, the equation

$$
f_{i}+\tau_{i j, j}=0 .
$$

Consequently, the volume integral in (18) vanishes identically. Introducing now in (18) a field $U_{i}$ that vanishes outside a compact set with an arbitrarily chosen 
nonempty intersection $\Sigma$ with $\partial \mathscr{D}$, we obtain

$$
\int_{\Sigma}\left(T_{i}-\tau_{i j} n_{j}\right) U_{i} d a=0
$$

Since $U_{i}$ itself can be chosen arbitrarily in the interior of $\Sigma$, we conclude that at each point of $\partial \mathscr{D}$ we must have necessarily that

$$
T_{i}=\tau_{i j} n_{j} .
$$

Equation (21) is the usual relation providing the stress vector in terms of the direction $\boldsymbol{n}$ of the exterior normal. Equation (20) is nothing but the classical equilibrium equation. Splitting in (19) symmetric and skew-symmetric parts, we can write

$$
\begin{aligned}
\tau_{[i j]}+C_{i j} & =0, \\
\tau_{i j}+\Phi_{i j} & =\sigma_{i j} .
\end{aligned}
$$

Up to this point we have always assumed that $\mathscr{D}$ is interior to $S$. For the sake of completeness, we should apply the principle of virtual power to $S$ itself. To this end, we will assume that the forces external to $S$ comprise, beyond the actions at a distance already mentioned, contact forces (whether known or unknown) defined by surface forces of density $t_{i}$. Reasoning just as before to obtain (21), we find the boundary condition that must be satisfied at each point of $\partial \mathscr{Y}$, namely,

$$
t_{i}=T_{i}=\tau_{i j} n_{j}
$$

In this equation, $n_{i}$ denotes the exterior unit normal at a point of $\partial \mathscr{Y}$, and $T_{i}$ denotes the stress vector for the direction $\boldsymbol{n}$ obtained by a passage to the limit, the point of $\partial \mathscr{S}$ being an accumulation point of a set of nearby points interior to $S$. The collection of the results obtained thus far can be summarized in the following statement.

Theorem 1. The necessary and sufficient conditions ensuring that the system $S$ is in equilibrium establish that the stress tensor satisfies Equations (20) and (22) at each interior point of $S$ and Equation (24) at each point of the boundary $\partial S$. Moreover, the intrinsic stress tensor and the volumetric energy of the internal forces are given, respectively, by (23) and (12).

Equations (20), (22), and (24) are those provided by the application of the fundamental law of conservation of linear momentum (Germain [3]); in addition, in that case, it is necessary to assume from the start that, at each point of $\partial \mathscr{D}, T_{i}$ is a function of $n_{j}$, an assumption which we did not need to invoke in the present treatment. On the other hand, this fundamental law cannot give us any information about the influence of the symmetric double forces that participate in the determination of the volumetric energy of the internal forces. Thus, even in the simple case of the first-gradient theory, it is not without interest to construct the general equations supplying the mechanical description of the system starting from the 
notion of virtual power. In the classical formulation of continuum mechanics, this advantage disappears, since in that case

$$
\Phi_{i j}=C_{i j}=0,
$$

so that

$$
\tau_{i j}=\sigma_{i j}
$$

The intrinsic stress tensor, therefore, coincides in this case with the stress tensor proper.

Remark. As we have already stated, we wanted to present the first-gradient theory in a systematic fashion. One can legitimately ask if, except for the classical case, this theory presents any physical interest. This point raises the question as to the physical meaning of the volumetric double forces, that is, the couples $C_{i j}$ and the symmetric double forces $\Phi_{i j}$. These forces can be properly interpreted if we assume that each material point of $S$ is equipped with a microstructure, and it is, in fact, very instructive to draw a correlation between the present theory and the theory of media endowed with microstructure, which we intend to do in a forthcoming article. At first sight, it may seem strange that the microstructure might participate at the level of the modeling of the external actions at a distance or that it might play any role in the modeling of the internal forces. It appears, however (see, for example, Lobdell [4]), that the first-gradient theory may be useful to describe certain electromechanical phenomena in solids.

Be that as it may, it is clear that this first-gradient theory is nothing but a slight extension of the classical theory, an extension that manifests itself in a nutshell in the formula (19). We only developed this theory here so as to show how to apply the virtual power method in a simple context in order to build a mechanical model of continuous media.

\section{Second-gradient theory}

The theory we are about to construct will be finer than the preceding one. We will consider as the space $\mathscr{V}$ of virtual motions the space of continuous and at least twice differentiable velocity fields defined on the closure $\mathscr{D}+\partial \mathscr{D}$ of $\mathscr{D}$, the norm in $\mathscr{V}$ being that of the uniform convergence for the velocities and their derivatives up to order 2 with respect to $x_{i}$. Our calculations will be analogous to those that can be found in [2] (see also [5;6]), but the interpretation given here is different and more comprehensive, and since our notation is not exactly the same as in those works, we believe that it is a good idea to repeat it here, at least in the Appendix, for the sake of assisting in the reading process. It is appropriate to choose a canonical representation of the (third-order) tensor of the second derivatives of $U_{i}$. Mindlin 
and Eshel [2] propose three different ones. We will content ourselves here with choosing the third one. Defining in the first place the rate of rotation vector $\omega_{i}$ as

$$
\omega_{i}=-\frac{1}{2} \varepsilon_{i p q} \Omega_{p q}, \quad \Omega_{i j}=-\varepsilon_{i j k} \omega_{k},
$$

we introduce the gradient tensor of the rate of rotation

$$
K_{i j}=\omega_{i, j}=-\frac{1}{2} \varepsilon_{i p q} \Omega_{p q, j}=-\frac{1}{2} \varepsilon_{i p q} U_{p, q j} .
$$

This tensor $K_{i j}$ is actually a deviator; that is, its trace $K_{i i}$ vanishes. It has, therefore, eight independent components. In the second place, we consider the completely symmetric part of the second gradient of the velocities

$$
K_{i j k}=\frac{1}{3}\left(U_{i, j k}+U_{j, k i}+U_{k, i j}\right) .
$$

The value of $K_{i j k}$ remains invariant under every permutation of the indices $i, j, k$. This tensor, therefore, has ten different components. The collection of the $K_{i j}$ and the $K_{i j k}$ determines completely the eighteen second derivatives $U_{i, j k}$ (and vice versa):

$$
U_{i, j k}=K_{i j k}-\frac{2}{3} \varepsilon_{i j l} K_{l k}-\frac{2}{3} \varepsilon_{i k l} K_{l j} .
$$

This relation can be easily established noting beforehand that, according to (26), we have

$$
\varepsilon_{l m i} K_{i j}=\frac{1}{2}\left(U_{l, m j}-U_{m, l j}\right) .
$$

5.1. We will start once again by formulating the virtual power of the internal forces, adopting evidently the working hypotheses stated in Section 3. Recalling the considerations developed in Section 4.1, and particularly formula (11), we see that, by virtue of the axiom, we can write the volumetric energy of the internal forces in the form

$$
p=\sigma_{i j} D_{i j}+\mu_{i j} K_{i j}+\mu_{i j k} K_{i j k} .
$$

The coefficients $\mu_{i j}$ are components of a second-order tensor, which is, incidentally, a deviator $\left(\mu_{i i}=0\right)$; the $\mu_{i j k}$ are components of a totally symmetric third-order tensor ( $\mu_{i j k}$ remains invariant under every permutation of the indices). These two tensors constitute a (canonical) representation of the intrinsic stresses of order 2 . All that is left now is to write $\mathscr{P}_{(i)}$ in the appropriate canonical form necessary to be able to apply the principle of virtual power. This is achieved proceeding, as in the preceding section, to carry out integrations by parts on the expression

$$
\mathscr{P}_{(i)}=-\int_{\mathscr{D}}\left(\sigma_{i j} D_{i j}+\mu_{i j} K_{i j}+\mu_{i j k} K_{i j k}\right) d v .
$$

For the first term, the integration by parts needs to be performed once; the result is the one obtained on the right-hand side of formula (13). For the remaining two terms, the calculation is slightly more complicated, since it is necessary to integrate 
twice; this is shown in the Appendix, and the results are those of formulas (A-11) and (A-9), respectively. We see, therefore, that we can write $\mathscr{P}_{(i)}$ in the form

$$
\mathscr{P}_{(i)}=\int_{\mathscr{D}} \mathscr{F}_{i} U_{i} d v+\int_{\partial \mathscr{D}}\left(\mathscr{T}_{i} U_{i}+\widetilde{\mathcal{M}} \widetilde{\omega}_{i}+\mathcal{N} D_{\underline{n} \underline{n}}\right) d a+\int_{\Gamma \curvearrowleft} \mathscr{R}_{i} U_{i} d s
$$

where we have put

$$
\left\{\begin{aligned}
\mathscr{F}_{i} & =\sigma_{i j, j}-\frac{1}{2} \varepsilon_{i p j} \mu_{p q, q j}-\mu_{i j k, j k}, \\
\mathscr{T}_{i} & =-\left(\sigma_{i j}-\frac{1}{2} \varepsilon_{i p j} \mu_{p q, q}-\mu_{i j k, k}\right) n_{j}+\frac{1}{2} \varepsilon_{i p j} D_{j}\left(\mu_{\underline{n} \underline{n}} n_{p}\right) \\
& \quad+\left(D_{j}-n_{j}\left(D_{p} n_{p}\right)\right)\left(\mu_{i j k} n_{k}+\mu_{j l k} n_{i} n_{l} n_{k}\right), \\
\tilde{M}_{i} & =2 \varepsilon_{i k q} \mu_{k j p} n_{j} n_{p} n_{q}-\left(\mu_{i q} n_{q}-n_{i} \mu_{\underline{n} \underline{n}}\right), \\
\mathcal{N} & =-\mu_{i j k} n_{i} n_{j} n_{k}, \\
\mathscr{R}_{i} & =-\llbracket \frac{1}{2} \delta_{i m} \mu_{\underline{n} \underline{n}}+\varepsilon_{j m q} n_{k} n_{q}\left(\mu_{i j k}+\mu_{p j k} n_{i} n_{p}\right) \rrbracket \tau_{m} .
\end{aligned}\right.
$$

The meaning of the symbols used in the formulas (32) is better given in the Appendix; $\widetilde{\omega}_{i}$ is the part of the rate of rotation vector $\omega_{i}$ tangential to $\partial \mathscr{D} ; D_{\underline{n} \underline{n}}$ and $\mu_{\underline{n n}}$ are real numbers representing the doubly normal component of the tensors $D_{i j}$ and $\mu_{i j}{ }^{*}$ The symbol $D_{i}$ is an operator of derivation tangential to the surface $\partial \mathscr{D}$, whose explicit expression is given in (A-2) (and, incidentally, $D_{p} n_{p}$ is nothing other than twice the mean curvature); $\Gamma$ denotes the edges of the boundary surface $\partial \mathscr{D}$ along which the tangent plane (or the normal vector $\boldsymbol{n}$ ) is discontinuous; $\tau_{i}$ is the unit vector tangent to $\Gamma$, whose orientation can be chosen arbitrarily, but consistently; finally, the symbol $\llbracket \rrbracket$ denotes the jump of the bracketed quantity across $\Gamma$. It is worthwhile noting that the sense across $\Gamma$ on which the jump takes place, and the sense of $\Gamma$ must be related, in agreement with the usual Stokes' formula.

It should be noted that the vector $\widetilde{M}_{i}$ is tangential to the surface $\partial \mathscr{D}$, since we have that $\mathscr{R}_{i} \widetilde{M}_{i}=0$. It is precisely this fact that the tilde is supposed to indicate.

5.2. We must presently formulate the expression of the virtual power of the external forces. If we want to proceed systematically, we must write the power of the actions at a distance, taking into consideration the remarks made in Section 3, in the form

$$
\mathscr{P}_{(d)}=\int_{\mathscr{D}}\left(f_{i} U_{i}+C_{i j} \Omega_{i j}+\Phi_{i j} D_{i j}+\Xi_{i j} K_{i j}+\Xi_{i j k} K_{i j k}\right) d v .
$$

On comparing (33) with (15), we perceive that there appear here additional forces, namely, the volumetric triple forces defined by $\Xi_{i j}$ - which is a deviatorand those defined by $\Xi_{i j k}$, which is a completely symmetric third-order tensor. In fact, we will assume, for the sake of simplicity, that these triple forces vanish;

*As indicated later, an underlined subscript nullifies the summation convention with respect to that subscript. - (TN) 
it should not be difficult to indicate how the expressions given below are to be modified if we want to take these forces into account. We will, therefore, accept that $\mathscr{P}_{(d)}$ is still given by (15) or, better, by (16), which is more suitable for the application of the principle of virtual power.

As far as the virtual power of the contact forces acting on the boundary surface $\partial \mathscr{D}$ is concerned, we are guided, as we were in the preceding section, to write the most suitable expression, by anticipating the application of the principle and taking into consideration the formula (31) already found for $\mathscr{P}_{(i)}$. We are thus led to write

$$
\mathscr{P}_{(c)}=\int_{\partial \mathscr{D}}\left(T_{i} U_{i}+\tilde{M}_{i} \widetilde{\omega}_{i}+N D_{\underline{n} \underline{n}}\right) d a+\int_{\Gamma \curvearrowleft} R_{i} U_{i} d s .
$$

As before, $T_{i}$ denotes the stress vector; $\widetilde{M}_{i}$, a vector tangent to $\partial \mathscr{D}$, is a surface density of a tangential couple; $N$ is a scalar surface density of a doubly normal double force; $R_{i}$ is a vector that defines a line density of a force applied along the edges of $\Gamma$.

5.3. It remains only to apply the principle of virtual power, that is, equality (9). We will substitute in it the expressions given in (16), (31), and (34); we thus obtain an equation that must be satisfied for every field $U_{i}$ twice continuously differentiable in the closure $\mathscr{D}+\partial \mathscr{D}$ of $\mathscr{D}$.

Let us consider first fields $U_{i}$ that vanish outside a compact set interior to $\mathscr{D}$. The only survivor is the volume integral that can be written as

$$
\int_{\mathscr{D}}\left(f_{i}+\tau_{i j, j}\right) U_{i} d v=0,
$$

if we set

$$
\tau_{i j}=\sigma_{i j}-\frac{1}{2} \varepsilon_{i p j} \mu_{p q, q}-\mu_{i j k, k}-C_{i j}-\Phi_{i j} .
$$

It follows that at each interior point of $\mathscr{D}$ we necessarily have

$$
f_{i}+\tau_{i j, j}=0 .
$$

In the equation expressing the principle of virtual power, therefore, the only remaining terms in the general case are the surface integral over $\partial \mathscr{D}$ and the line integral over the edges $\Gamma$. Let us consider a fixed arbitrary closed connected area $\Sigma$ which is a subset of $\partial \mathscr{D}$ not having any point in common with an edge, and let us denote by $C_{2}(\Sigma)$ the collection of twice continuously differentiable scalar-valued functions defined over $\Sigma$ and vanishing outside a compact set interior to $\Sigma$. We state:

Lemma. Given seven functions in $C_{2}(\Sigma)-V_{1}(P), V_{2}(P), V_{3}(P), \widetilde{\Omega}_{1}(P), \widetilde{\Omega}_{2}(P)$, $\widetilde{\Omega}_{3}(P), D(P)$-constrained by the single relation $n_{i} \widetilde{\Omega}_{i}=0$, it is possible to construct a twice continuously differentiable field $U_{i}$ on the closure $\mathscr{D}+\partial \mathscr{D}$ attaining 
on the boundary $\partial \mathscr{D}$ the following values:

$$
\begin{aligned}
\text { at each point of } \Sigma, & U_{i}=V_{i}, \widetilde{\omega}_{i}=\widetilde{\Omega}_{i}, \quad D_{\underline{n} \underline{n}}=D, \\
\text { at all other points of } \partial \mathscr{D}, & U_{i}=\widetilde{\Omega}_{i}=D_{\underline{n} \underline{n}}=0 .
\end{aligned}
$$

The proof is straightforward. The velocity field that we are trying to construct has, according to equalitites (37), known values on $\Sigma$ and a gradient that also has known values on $\Sigma$ (the tangential derivatives are determined by those of $V_{i}$, the normal derivatives of the tangential components are next determined by $\widetilde{\Omega}_{i}$, and the normal derivative of the normal component by $D$ ). Let us consider the subset $\Delta$ of $\mathscr{D}$ made up of the points $M$ such that $\boldsymbol{M P}=\zeta \boldsymbol{n}$, with $0 \leq \zeta \leq \zeta_{0}(P)$, where $\zeta_{0}(P)$ is a function defined over $\Sigma$ and infinitely differentiable on $\Sigma$ vanishing on the boundary and attaining at each point of $\Sigma$ sufficiently small values so that at each point of $\Delta$ there is a unique normal to $\Sigma$. It is clear that we can construct in $\Delta$ a field $U_{i}(M)=U_{i}(P, \zeta)$ such that it and its first derivatives attain the values prescribed on $\Sigma$ and also such that it and its derivatives up to order 2 vanish over the part of $\partial \Delta-\Sigma$ of the boundary of $\Delta$. Thus, in a trivial fashion, we complete the definition of $U_{i}$ by assigning to it zero values on the set $\mathscr{D}+\partial \mathscr{D}-\Delta$, and this field satisfies perfectly the conditions of the lemma.

Applying the equation of virtual power to such a field $U_{i}$ yields

$$
\int_{\Sigma}\left\{\left(T_{i}+\mathscr{T}_{i}+C_{i j} n_{j}+\Phi_{i j} n_{j}\right) V_{i}+\left(\tilde{M}_{i}+\widetilde{M}_{i}\right) \widetilde{\Omega}_{i}+(N+\mathcal{N}) D\right\} d a=0,
$$

for arbitrary functions $V_{i}, \widetilde{\Omega}_{i}, D$, constrained by the single relation $n_{i} \widetilde{\Omega}_{i}=0$. Furthermore, since the vector $\widetilde{M}_{i}+\widetilde{M}_{i}$ is a vector tangent to the surface $\Sigma$, the quantities within the parentheses ( ) under the integral sign must vanish individually at each point of $\Sigma$ and, consequently, taking into account the latitude with which $\Sigma$ can be chosen, also at each point of the boundary $\partial \mathscr{D}$ not belonging to an edge. In accordance with (32), we can write

$$
\left\{\begin{aligned}
T_{i} & =\tau_{i j} n_{j}+T_{i}^{\prime}=\widehat{T}_{i}+T_{i}^{\prime}, \\
T_{i}^{\prime} & =\left(n_{j}\left(D_{p} n_{p}\right)-D_{j}\right)\left(\mu_{i j k} n_{k}+\mu_{l j k} n_{i} n_{l} n_{k}\right)-\frac{1}{2} \varepsilon_{i p j} D_{j}\left(n_{p} \mu_{\underline{n} \underline{n}}\right), \\
\widetilde{M}_{i} & =\mu_{i q} n_{q}-n_{i} \mu_{\underline{n} \underline{n}}-2 \varepsilon_{i k q} \mu_{k j p} n_{j} n_{p} n_{q}, \\
N & =\mu_{i j k} n_{i} n_{j} n_{k} .
\end{aligned}\right.
$$

Let us note that the last term appearing in the expression of $T_{i}^{\prime}$ also can be written as in (A-12), that is,

$$
-\frac{1}{2} \varepsilon_{i p j} D_{j}\left(n_{p} \mu_{\underline{n} \underline{n}}\right)=-\frac{1}{2} \varepsilon_{i p j} n_{p} \mu_{\underline{n} \underline{n}, j} .
$$

These equations show how the stress vector $T_{i}$, the surface tangential couple $\tilde{M}_{i}$, and the (surface) normal double force $N$ are expressed in terms of the intrinsic 
stress tensors of orders 1 and 2 . We can still call $\tau_{i j}$ the stress tensor, given that the equilibrium equation has the usual form, but it must be noted that in the present case this tensor is no longer sufficient to define the stress vector $T_{i}$. Finally, taking into consideration the results already obtained, the equality of virtual powers reduces to

$$
\int_{\Gamma}\left(R_{i}+\mathscr{R}_{i}\right) U_{i} d s=0,
$$

and therefore, taking (32) into account, we have

$$
R_{i}=\llbracket \frac{1}{2} \delta_{m i} \mu_{\underline{n} \underline{n}}+\varepsilon_{j m q} n_{k} n_{q}\left(\mu_{i j k}+\mu_{p j k} n_{i} n_{p}\right) \rrbracket \tau_{m},
$$

an equation that allows us to express the line force along the edges as a function of the intrinsic stress tensors.

In addition, it remains to apply the principle to $S$ itself in order to find the boundary conditions. To this end, we are led to admit that the external forces exerted on $S$ comprise, beyond the volume actions at a distance already considered, surface effects defined by surface forces of density $t_{i}$, couples tangential to $S$ of surface density $\tilde{m}_{i}$, and doubly normal double forces of surface density $n$. We can easily show that we must have

$$
\begin{cases}T_{i}=t_{i}, \tilde{M}_{i}=m_{i}, N=n & \text { on } \partial S, \\ R_{i}=r_{i} & \text { on } \Gamma .\end{cases}
$$

We have thus exhausted the consequences that can be extracted from the principle of virtual power, and we can consequently establish the following:

Theorem 2. The necessary and sufficient conditions ensuring that the system $S$ is in equilibrium, for the case in which the external triple volume forces are neglected, are expressed by the relations (35) and (36), that must be satisfied at each interior point of $S$, and the relations (40), that must be satisfied at each point of the boundary $\partial S$. Moreover, in the interior of $S$, the surface contact forces are defined by (38) and the line forces by (39). When the external triple volume forces are taken into consideration, the fundamental equations of statics of the secondgradient theory are obtained by replacing in the previous equations $\mu_{i j}$ and $\mu_{i j k}$ by $\mu_{i j}-\Xi_{i j}$ and $\mu_{i j k}-\Xi_{i j k}$, respectively.

5.4. It is instructive to interpret the preceding results in terms of the classical fundamental law. In so doing, we will discover the extra information and precision contributed by the formulation herein advocated, which turns out to be better suited when we are dealing with a medium for which the modeling must be finer than in the classical case.

Let us recall a remark already made above: the statements purveying the fundamental law can be obtained from the equations expressing the principle of virtual 
power by considering only the rigidifying virtual motions of $\mathscr{D}$, for which the velocity field is determined by a twist, that is, a velocity field of the form

$$
U_{i}=V_{i}+\varepsilon_{i k j} \bar{\omega}_{k} x_{j},
$$

where $\bar{\omega}_{i}$ and $V_{i}$ are the elements of reduction of the twist at the origin.

Here is a first application. Let us take up again the expression of the virtual power of the internal forces given by (30), and instead of proceeding to carry out two integrations by parts to end up with (31), let us retain the intermediate result obtained after a single integration. Setting

$$
\beta_{i j}=\sigma_{i j}-\frac{1}{2} \varepsilon_{i p j} \mu_{p q, q}-\mu_{i j k, k},
$$

that is, according to (35),

$$
\tau_{i j}=\beta_{i j}-C_{i j}-\Phi_{i j}
$$

we can write

$$
0=\int_{\mathscr{D}} \beta_{i j, j} U_{i} d v-\int_{\partial \mathscr{D}} \beta_{i j} n_{j} U_{i} d a+\int_{\partial \mathscr{D}}\left(\frac{1}{2} \varepsilon_{i j p} \mu_{p q} n_{q} U_{i, j}-\mu_{i j k} n_{k} U_{i, j}\right) d a .
$$

Let us apply this equation using the field (41); the term in $\mu_{i j k}$ does not contribute at all, by virtue of the symmetry with respect to the first two indices. If we set

$$
\gamma_{i}=\mu_{i j} n_{j}, \quad\left(\gamma_{i}\right)_{\partial \mathscr{D}}=\int_{\partial \mathscr{D}} \gamma_{i} d a,
$$

where $\left(\gamma_{i}\right)_{\partial \mathscr{D}}$ denotes the couple defined by the surface density of the couples $\gamma_{i}$ on $\partial \mathscr{D}$, we immediately obtain, using the notations introduced above,

$$
\left[\beta_{i j, j}\right]_{\mathscr{D}}=\left[\beta_{i j} n_{j}\right]_{\partial \mathscr{D}}+\left(\gamma_{i}\right)_{\partial \mathscr{D}} .
$$

With the same notation, an equation of the form

$$
\int_{\partial \mathscr{D}} \tau_{i j} n_{j} U_{i} d a=\int_{\mathscr{D}} \tau_{i j, j} U_{i} d v+\int_{\mathscr{D}} \tau_{i j} U_{i, j} d v,
$$

when applied to the field (41), leads to the wrench equation

$$
\left[\tau_{i j} n_{j}\right]_{\partial \mathscr{D}}=\left\{\left[\tau_{i j, j}\right]_{\mathscr{D}}+\left(\varepsilon_{k i j} \tau_{k j}\right)_{\mathscr{D}}\right\}=0 .
$$

We have, therefore,

$$
\left[\Phi_{i j, j}\right]_{\mathscr{D}}=\left[\Phi_{i j} n_{j}\right]_{\partial \mathscr{D}}, \quad\left[C_{i j, j}\right]_{\mathscr{D}}=\left[C_{i j} n_{j}\right]_{\mathscr{D}}-\left(c_{i}\right)_{\mathscr{D}},
$$

where we have set

$$
c_{k}=-\varepsilon_{k i j} C_{i j} .
$$

The vector $c_{i}$ is the couple vector associated with the skew-symmetric tensor $C_{i j}$. Combining (42), (43), and (45), we can formulate the following: 
Proposition 3. For every part $\mathscr{D}$ of $S$, we have the following wrench equation:

$$
\left[\tau_{i j, j}\right]_{\mathscr{D}}-\left\{\left[\tau_{i j} n_{j}\right]_{\partial \mathscr{D}}+\left(\gamma_{i}\right)_{\partial \mathscr{D}}+\left(c_{i}\right)_{\mathscr{D}}\right\}=0 .
$$

Recall that $\widehat{T}_{i}=\tau_{i j} n_{j}$.

Furthermore, when applied to a twist such as (41), since $\mathscr{P}_{(i)}$ vanishes, $\mathscr{P}_{(d)}$ and $\mathscr{P}_{(c)}$ being given by (15) and (34), respectively, the statement of the principle of virtual power leads to the wrench equation

$$
\left[f_{i}\right]_{\mathscr{D}}+\left(c_{i}\right)_{\mathscr{D}}+\left[\widehat{T}_{i}\right]_{\partial \mathscr{D}}+\left[T_{i}^{\prime}\right]_{\partial \mathscr{D}}+\left(\tilde{M}_{i}\right)_{\partial \mathscr{D}}+\left[R_{i}\right]_{\Gamma}=0 .
$$

Let us add (47) and (48), and let us take (36) into account to obtain

$$
\left(\gamma_{i}\right)_{\partial \mathscr{D}}=\left[T_{i}^{\prime}\right]_{\partial \mathscr{D}}+\left(\tilde{M}_{i}\right)_{\partial \mathscr{D}}+\left[R_{i}\right]_{\Gamma} .
$$

The right-hand side must be, just as the left-hand side, a couple, which implies in particular that the resultant of the wrench that it defines vanishes, namely,

$$
\int_{\partial \mathscr{D}} T_{i}^{\prime} d a+\int_{\Gamma} R_{i} d s=0
$$

Taking (49) into account, we can write (48) in the form

$$
\left[f_{i}\right]_{\mathscr{D}}+\left(c_{i}\right)_{\mathscr{D}}+\left[\widehat{T}_{i}\right]_{\partial \mathscr{D}}+\left(\gamma_{i}\right)_{\partial \mathscr{D}}=0 .
$$

The first two terms of the left-hand side represent the wrench of the actions at a distance exerted on $\mathscr{D}$ - the double forces $\Phi_{i j}$ must be considered as defining a zero wrench, since their virtual power vanishes in every rigidifying motion. It follows that the last two terms represent the wrench of the contact actions. Consequently, we obtain the following:

Proposition 4. On every part $\mathscr{D}$ of $S$ the contact actions determine a wrench that can be defined by a surface force $\widehat{T}_{i}$ and a surface couple $\gamma_{i}$ which are linear functions of the unit normal vector $\boldsymbol{n}$. We have indeed

$$
\widehat{T}_{i}=\tau_{i j} n_{j}, \quad \gamma_{i}=\mu_{i j} n_{j} .
$$

This representation of the contact forces is in appearance simpler than that given in Section 5.2; but this is no more than a globally valid representation. For this reason, in the absence of supplementary details, this representation is in effect insufficient for the study of media endowed with stress couples, such as those that fall under the scope of the second-gradient theory. Let us, moreover, recall that by means of a statement such as that of the classical fundamental law appearing in equality (50) it is not possible to take into account the external double forces. Finally, we should note that, adding (44) and (47), we obtain

$$
\left(\varepsilon_{k i j} \tau_{k j}\right)_{\mathscr{D}}+\left(\gamma_{i}\right)_{\partial \mathscr{D}}+\left(c_{i}\right)_{\mathscr{D}}=0,
$$


and using equality (51), transforming the surface integral into a volume integral, and considering (46), we find in the end, since $\mathscr{D}$ is an arbitrary part of $S$, the equation

$$
\tau_{[i j]}=-\frac{1}{2} \varepsilon_{i p j} \mu_{p q, q}-C_{i j},
$$

expressing the equality of the skew-symmetric parts of the two sides of (35).

5.5. The representation adopted for the second-gradient (26) and (27) allows us to deal immediately with the case where $K_{i j k}$ does not participate in the virtual power. Indeed, it suffices to set $\mu_{i j k}=0$ in the previous formulas. For example, if we consider (38) and (39), we notice that $N=0$ and that, more precisely, we have

$$
\begin{gathered}
T_{i}=\tau_{i j, j}+T_{i}^{\prime}=\widehat{T}_{i}+T_{i}^{\prime}, \quad T_{i}^{\prime}=-\frac{1}{2} \varepsilon_{i p j} D_{j}\left(n_{p} \mu_{\underline{n} \underline{n}}\right), \\
\tilde{M}_{i}=\mu_{i q} n_{q}-n_{i} \mu_{\underline{n} \underline{n}}=\left(\widetilde{\mu_{i q} n_{q}}\right)=\tilde{\gamma}_{i}, \\
R_{i}=\frac{1}{2} \mu_{n n} \tau_{i} .
\end{gathered}
$$

The notations of (51) have been used. Naturally, we have

$$
\tau_{i j}=\left(\sigma_{i j}-\Phi_{i j}\right)+\left(\frac{1}{2} \varepsilon_{i j p} \mu_{p q, q}-C_{i j}\right) .
$$

On the right-hand side, the first term in parentheses is the even part of $\tau_{i j}$ - which reduces to $\sigma_{i j}$ if we neglect the volumetric double forces - while the second term in brackets is the odd part.

The boundary conditions associated naturally to this model are still given by (40), on condition that the equation $N=n$ be omitted, since it is superfluous here. It is precisely these conditions that can suggest the boundary conditions to take into consideration to formulate problems with regular boundaries. We leave to the reader the effort of particularizing the results given in Sections 6 and 7 for this simplified case.

5.6. A final remark. We have not treated here either the dynamical case or the case of equations with discontinuities. We intend to revisit this topic in a forthcoming article in which we will specify in advance the relations between the secondgradient theory and the theory of media endowed with microstructure. The physical meaning of the first will then be more easily brought to light.

\section{Constitutive behavior of a medium under the umbrella of the second-gradient theory within the framework of small perturbations. The case of elastic media}

To study the response of a system $S$ under the action of external agents, it is necessary to supplement the general equations obtained above by means of constitutive equations. In this section, we intend to briefly examine how this can be achieved 
within the framework of problems that can be treated under the hypothesis of small strains.

Under these conditions, indeed, there is no need to distinguish between the Lagrangian and Eulerian representations. The motion of the medium is defined by the displacement field $X_{i}\left(x_{1}, x_{2}, x_{3}, t\right)$ defined over a well determined domain $S$, which plays the role of a reference configuration. The strains for a second-gradient theory can then be fully characterized by the tensors

$$
\begin{aligned}
\varepsilon_{i j} & =\frac{1}{2}\left(X_{i, j}+X_{j, i}\right), \\
\eta_{i j} & =-\frac{1}{2} \varepsilon_{i p q} X_{p, q j} \quad\left(\eta_{i i}=0\right), \\
\eta_{i j k} & =\frac{1}{3}\left(X_{i, j k}+X_{j, k i}+X_{k, i j}\right),
\end{aligned}
$$

since, under the hypothesis of small perturbations, the material derivative being identical in this case to the partial derivative with respect to time, the strain-rate tensors of the second-gradient theory are obtained by simple differentiation. We have, therefore (see (26), (27)):

$$
D_{i j}=\dot{\varepsilon}_{i j}, \quad K_{i j}=\dot{\eta}_{i j}, \quad K_{i j k}=\dot{\eta}_{i j k} .
$$

6.1. The strain energy. Let us suppose now that the medium is elastic, and the evolution is isothermal. The existence of the free-energy density implies that of a volumetric strain energy having the usual convexity properties. More precisely, we will state, in view of the small-perturbation hypothesis, the following:

Definition 1. There exists a volumetric strain energy $\mathrm{w}\left(\varepsilon_{i j}, \eta_{i j}, \eta_{i j k}\right)$, depending on the variables $\varepsilon_{i j}, \eta_{i j}, \eta_{i j k}$, which is a nonnegative quadratic form, invariant under any permutation of the two indices of the variable $\varepsilon_{i j}$, under any permutation of the three indices of $\eta_{i j k}$, and under the addition of the same constant to the three variables $\eta_{11}, \eta_{22}, \eta_{33}$, which vanishes if, and only if,

$$
\varepsilon_{i j}=0, \quad \eta_{i j k}=0, \quad \eta_{i j}=c \delta_{i j} .
$$

Moreover, for every motion, the derivative with respect to time of $\mathrm{w}$ is equal to the volumetric energy $p$ of the internal forces.

It follows from this definition that the function $w$ satisfies the equation

$$
\frac{\partial \mathrm{w}}{\partial \eta_{11}}+\frac{\partial \mathrm{w}}{\partial \eta_{22}}+\frac{\partial \mathrm{w}}{\partial \eta_{33}}=0,
$$

and that, if the strain tensors are defined in terms of a displacement field by the formulas (52), (53), and (54), w vanishes if, and only if, the field $X_{i}$ is given by a twist, that is, a geometric infinitesimal rigid-body displacement. 
Furthermore, in a given state $\varepsilon_{i j}, \eta_{i j}, \eta_{i j k}$, it is possible to assign to the strainrate tensors (55) arbitrary values subject to the single restriction

$$
\dot{\eta}_{i i}=0 \text {. }
$$

According to the definition, if $\lambda$ denotes a Lagrange multiplier, we have identically that

$$
\dot{\mathrm{w}}=\sigma_{i j} \dot{\varepsilon}_{i j}+\mu_{i j} \dot{\eta}_{i j}+\mu_{i j k} \dot{\eta}_{i j k}+\lambda \dot{\eta}_{i i}
$$

and, therefore, necessarily that

$$
\sigma_{i j}=\frac{\partial \mathrm{w}}{\partial \varepsilon_{i j}}, \quad \mu_{i j}=\frac{\partial \mathrm{w}}{\partial \eta_{i j}}, \quad \mu_{i j k}=\frac{\partial \mathrm{w}}{\partial \eta_{i j k}},
$$

since, by virtue of (56) and of the relation $\mu_{i i}=0$, the Lagrange multiplier $\lambda$ is necessarily zero.

Equations (59) are the constitutive laws of the elastic medium.

In order to simplify the notation, we will denote by $c$ the collection of the tensors $\varepsilon_{i j}, \eta_{i j}\left(\eta_{i i}=0\right), \eta_{i j k}$, and by $\sigma$ the collection of the tensors $\sigma_{i j}, \mu_{i j}\left(\mu_{i i}=0\right), \mu_{i j k}$. We will write the strain energy in the form $\mathrm{w}(c)$ and the constitutive laws (59) as

$$
\sigma=\frac{\partial \mathrm{w}(c)}{\partial c}=E(c) .
$$

With these notations, Euler's identity for w attains the form

$$
2 \mathrm{w}=\sigma \cdot c .
$$

The general form of $w$ involves 300 coefficients. But this number is reduced to seven in the case of isotropic media. (See, for example, Mindlin [5], Mindlin and Eshel [2].)

All the results of the classical theory of linear elasticity (see, for example, Germain [7]) are easily extended to the case of the present theory. To this end we introduce the following notations:

- bilinear form $w$ associated to $\mathrm{w}(c)$ :

$$
\mathrm{w}\left(c+c^{*}\right)=\mathrm{w}(c)+2 w\left(c, c^{*}\right)+\mathrm{w}\left(c^{*}\right),
$$

- strain energy of the system $S$ and associated bilinear form:

$$
W(C)=\int_{S} \mathrm{w}(c) d v, \quad \mathscr{W}\left(C, C^{*}\right)=\int_{S} w\left(c, c^{*}\right) d v .
$$

Here, $C$ denotes a strain tensor field $c$ defined over $S$. 
- Dual notions:

$$
\begin{aligned}
\mathrm{w}^{*}(\sigma) & =\sup _{c}\{\sigma \cdot c-\mathrm{w}(c)\}, \\
\mathrm{w}^{*}\left(\sigma+\sigma^{*}\right) & =\mathrm{w}^{*}(\sigma)+2 w^{*}\left(\sigma, \sigma^{*}\right)+\mathrm{w}^{*}\left(\sigma^{*}\right), \\
W^{*}(\Sigma) & =\int_{S} \mathrm{w}(\sigma) d v, \quad \mathcal{W}^{*}\left(\Sigma, \Sigma^{*}\right)=\int_{L} w\left(\sigma, \sigma^{*}\right) d v_{0} .
\end{aligned}
$$

Obviously, $\mathrm{w}^{*}(\sigma)$ is a quadratic form of $\sigma$ having invariance properties analogous to those of w $(c) ; \Sigma$ denotes a field of stress tensors $\sigma$ defined on $S$.

The constitutive equations can be written as

$$
c=\frac{\partial \mathrm{w}^{*}(\sigma)}{\partial \sigma}=E^{-1}(\sigma),
$$

where $E^{-1}$ denotes the inverse function of $E(c)$, given in (60).

It should be noted that

$$
\mathrm{w}^{*}(\sigma)=\mathrm{w}(c) \text { if } \sigma=E(c) .
$$

6.2. The energy theorems. Let $\Sigma$ be a field of intrinsic stresses $\sigma\left(\sigma_{i j}, \mu_{i j}, \mu_{i j k}\right)$, in equilibrium with given external forces $\mathscr{F}\left(f_{i}, C_{i j}, \Phi_{i j}, t_{i}, \widetilde{m}_{i}, n, r_{i}\right)$ defined as in Section 5 (see (15), (34), and (40)). If $\mathscr{C}^{*}$ denotes the virtual motion defined by the twice continuously differentiable field $X_{i}^{*}$ and the corresponding strain-rate tensors, $\varepsilon_{i j}^{*}, \eta_{i j}^{*}, \eta_{i j k}^{*}$ according to the formulas (52), (53), (54), as well as the rotation rates

$$
\varphi_{i j}^{*}=\frac{1}{2}\left(X_{i, j}^{*}-X_{j, i}^{*}\right), \quad \varphi_{i}^{*}=-\frac{1}{2} \varepsilon_{i j k} \varphi_{j k}^{*},
$$

the virtual power of the forces $\mathscr{F}$ in the virtual motion $\mathscr{C}^{*}$ can be written as

$$
\begin{aligned}
\left\langle\mathscr{F}, \mathscr{C}^{*}\right\rangle=\int_{S}\left(f_{i} X_{i}^{*}+C_{i j} \varphi_{i j}^{*}\right. & \left.+\Phi_{i j} \varepsilon_{i j}^{*}\right) d v \\
& +\int_{\partial \mathscr{D}}\left(t_{i} X_{i}^{*}+\widetilde{m}_{i} \widetilde{\varphi}_{i}^{*}+n \varepsilon_{\underline{n n}}^{*}\right) d a+\int_{\Gamma} r_{i} X_{i}^{*} d s .
\end{aligned}
$$

We can, therefore, state:

Proposition 5. If the elastic medium $S$ is in equilibrium under the action of external

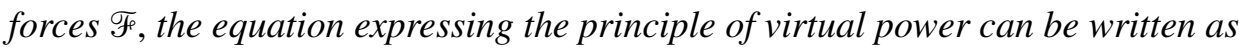

$$
\left\langle\mathscr{F}, \mathscr{C}^{*}\right\rangle=2 \mathcal{W}\left(C, C^{*}\right),
$$

where $C$ is the field of strains $c$ of the medium $S$ in elastic equilibrium.

Indeed, if $\sigma=E(c)$, then $2 w\left(c, c^{*}\right)=\sigma \cdot c^{*}$. Analogously:

Proposition 6. If a stress field $\widetilde{\Sigma}$ is in equilibrium with external forces $\widetilde{\mathscr{F}}$, and if $\mathscr{C}(C), \Sigma$ denote, respectively, a field of displacements, rotations, and strains, and a field of stresses of an elastic equilibrium state, then

$$
\langle\widetilde{\mathscr{F}}, \mathscr{b}\rangle=2 W^{*}(\Sigma, \widetilde{\Sigma}) \text {. }
$$


These two propositions furnish an interesting interpretation of the functions $\mathcal{W}$ and $\mathscr{W}^{*}$.

It is not necessary to dwell on showing how the work and reciprocity theorems are derived. We leave their formulation as an exercise to the reader. It is a straightforward matter also to obtain the uniqueness theorem for regular problems.

To avoid a complicated notation and following the current usage, we will examine a problem of the following type (type III). Let $\Sigma_{1}$ and $\Sigma_{2}$ be two disjoint parts of the boundary $\partial S$ of $S$. The problem data are

(a) $f_{i}, C_{i j}, \Phi_{i j}$ in $S$,

(b) on $\Sigma_{1}, X_{i}=\bar{X}_{i}, \widetilde{\varphi}_{i}=\overline{\widetilde{\varphi}}_{i}, \varepsilon_{\underline{n} \underline{n}}=\bar{\varepsilon}_{\underline{n} \underline{n}}$,

(c) on $\Sigma_{2}, t_{i}=\bar{t}_{i}, \widetilde{m}_{i}=\overline{\widetilde{m}}_{i}, n=\bar{n}$, and $r_{i}=\bar{r}_{i}$ on $\Gamma \cap \Sigma_{2}$.

In other words, we are given in $S$ and on $\Sigma_{2}$ the external forces and on $\Sigma_{1}$ the displacements, the tangential rotation, and the unitary elongation in the normal direction. We then have (Mindlin and Eshel [2]):

Theorem 3. The problem thus posed has at most one nontrivial solution.

Indeed, applying (68) to the homogeneous problem associated with $\mathscr{C}^{*}=\mathscr{C}$, we have, by virtue of the data of this homogeneous problem, $\langle\mathscr{F}, \mathscr{C}\rangle=0$ and, therefore, $W(C)=0$, which implies that, at every point of $S, \mathrm{w}(c)=0$, since $\mathrm{w}(c)$ is nonnegative and continuous. Consequently, the displacement field is a twist, and the strain and stress fields vanish identically. These properties are precisely what characterizes a trivial solution. We can also give a variational formulation of the regular problems. We will so do in the case of the problem formulated above. To this end, let us propose the following definitions.

Definition 2. A field $\mathscr{C}^{\prime}$, determined by the field $X_{i}^{\prime}\left(x_{1}, x_{2}, x_{3}\right)$, assumed to be defined and twice differentiable in the closure of $S$, is said to be kinematically admissible for the problem under consideration if it satisfies the kinematic boundary conditions on $\Sigma_{1}$ (condition (b)). ${ }^{*}$ We will denote by $C^{\prime}$ the strain field defined by $\mathscr{b}$.

Definition 3. A field $\Sigma^{\prime}$ determined by the tensor fields $\sigma_{i j}^{\prime}, \mu_{i j}^{\prime}\left(\mu_{i i}^{\prime}=0\right), \mu_{i j k}^{\prime}$ defined and twice differentiable in the closure of $S$ is said to be statically admissible for the problem under consideration if it satisfies the equilibrium equations (35) and (36) in the interior of $S$ and the boundary conditions (38) and (40) on $\Sigma_{2}$ and on $\Gamma \cap \Sigma_{2}$.

Let us remark that if we denote by $\overline{\mathscr{F}}$ the data of external forces defined in $S$ and on $\Sigma_{2}$, by $\overline{\mathscr{C}}$ the kinematic data on $\Sigma_{1}$, and by $\mathscr{F}^{\prime}$ the system of external forces

${ }^{*}$ The original has $\Sigma_{2}$ instead of $\Sigma_{1} \cdot-(\mathrm{TN})$ 
in equilibrium with $\Sigma^{\prime}$, we can write

$$
\left\langle\mathscr{F}^{\prime}, \mathscr{C}^{\prime}\right\rangle=\left\{\overline{\mathscr{F}}, \mathscr{C}^{\prime}\right\}+\left(\mathscr{F}^{\prime}, \overline{\mathscr{C}}\right),
$$

where \{\} collects in (68) the integrals taken on $S$ and on $\Sigma_{2}$, while () collects the integrals on $\Sigma_{1}$.

We can still formulate the following definitions.

Definition 4. We call the potential energy of a kinematically admissible field for the problem under consideration the function

$$
V\left(C^{\prime}\right)=W\left(C^{\prime}\right)-\left\{\overline{\mathscr{F}}, C^{\prime}\right\},
$$

and the potential energy of a statically admissible field for the problem under consideration the function

$$
V^{*}\left(\Sigma^{\prime}\right)=-W\left(\Sigma^{\prime}\right)+\left(\mathscr{F}^{\prime}, \overline{\mathscr{C}}\right) .
$$

We can then prove the following:

Theorem 4. Suppose that the problem under consideration admits a solution. If $C$ and $\Sigma$ denote the strain and stress fields defining this solution, we have that for every field of admissible $\mathscr{C}^{\prime}$ and $\sigma^{\prime}$

$$
V^{*}\left(\Sigma^{\prime}\right) \leq V^{*}(\Sigma)=V(C) \leq V\left(C^{\prime}\right) .
$$

Moreover, it is not possible to have the equality $V\left(C^{\prime}\right)=V(C)$ except if $C$ and $C^{\prime}$ define the same strain tensor fields, and it is not possible to have $V^{*}\left(\Sigma^{\prime}\right)=V(\Sigma)$ except if $\Sigma^{\prime}$ and $\Sigma$ define the same intrinsic stress fields.

The proof is easy and classic. Let us set $\mathscr{C}^{\prime}=\mathscr{C}+\mathscr{C}^{*}$. Then, $C^{\prime}=C+C^{*}$ and, with obvious notation,

$$
V\left(C^{\prime}\right)-V(C)=W\left(C+C^{*}\right)=W(C)-\left\{\overline{\mathscr{F}}, \mathscr{b}^{*}\right\} .
$$

By virtue of (62) and (63),

$$
W\left(C+C^{*}\right)-W(C)=2 W\left(C, C^{*}\right)+W\left(C^{*}\right) .
$$

On the other hand, according to (71), for every $\mathscr{F}^{\prime}$, and in particular for $\mathscr{F}$ corresponding to the solution,

$$
\left\langle\mathscr{F}^{\prime}, \mathscr{C}^{*}\right\rangle=\left\langle\mathscr{F}^{\prime}, \mathscr{C}^{\prime}\right\rangle-\left\langle\mathscr{F}^{\prime}, \mathscr{C}\right\rangle=\left\{\overline{\mathscr{F}}, \mathscr{C}^{*}\right\}=\left\langle\mathscr{F}, \mathscr{C}^{*}\right\rangle ;
$$

and finally, according to (69), $\mathscr{C}$ and $\mathscr{F}$ being associated with the solution,

$$
\left\langle\mathscr{F}, \mathscr{C}^{*}\right\rangle=2 \mathcal{W}\left(C, C^{*}\right) .
$$


Plugging these results into (75) yields

$$
V\left(C^{\prime}\right)-V(C)=W\left(C^{*}\right) .
$$

The right-hand side of (76) is always nonnegative, and it doesn't vanish except when the strain tensors defined by $C$ and $C^{\prime}$ are identical. Let us set $\Sigma^{\prime}=\Sigma+\widetilde{\Sigma}$ and, accordingly, $\mathscr{F}^{\prime}=\mathscr{F}+\widetilde{\mathscr{F}}$. Then

$$
V^{*}\left(\Sigma^{\prime}\right)-V^{*}(\Sigma)=W^{*}(\Sigma+\widetilde{\Sigma})-W^{*}(\Sigma)-(\widetilde{\mathscr{F}}, \overline{\mathscr{C}}) .
$$

But

$$
W^{*}(\Sigma+\widetilde{\Sigma})-W^{*}(\Sigma)=2^{*}(\Sigma, \widetilde{\Sigma})+W^{*}(\widetilde{\Sigma})
$$

Moreover, according to (71), for every admissible $\mathscr{C}^{\prime}$ and in particular for the solution $\mathscr{C}$,

$$
\left\langle\tilde{\mathscr{F}}^{*}, \mathscr{C}^{\prime}\right\rangle=\left\langle\mathscr{F}^{\prime}, \mathscr{C}^{\prime}\right\rangle-\left\langle\mathscr{F}^{\prime}, \mathscr{C}^{\prime}\right\rangle=(\tilde{\mathscr{F}}, \tilde{\mathscr{C}})=\langle\mathscr{F}, \mathscr{C}\rangle
$$

and finally, according to (70)

$$
\langle\widetilde{\mathscr{F}}, \mathscr{C}\rangle=2 \mathscr{W}^{*}(\Sigma, \widetilde{\Sigma}) .
$$

It follows then that

$$
V^{*}(\Sigma)-V^{*}\left(\Sigma^{\prime}\right)=W(\widetilde{\Sigma})
$$

which establishes the first inequality (74).

Finally, quite evidently by virtue of (69) or of (70) or, more directly, by the work theorem, we have for the solution $\mathscr{C}, \Sigma$

$$
V(C)=V^{*}(\Sigma) .
$$

This completes the proof of the energy theorem.

6.3. Hints on the existence theorems. It is not difficult to extend most of the theorems of existence established in the classical theory (Duvaut-Lions [1], Ch. 3) to the problems of linear elasticity formulated within the framework of the secondgradient theory. We will content ourselves here with the formulation of one of these theorems, always in the case of the problem of type III considered above, assuming, moreover, for the sake of simplicity, on the one hand, that the boundary $\partial S$ of $S$ is a twice continuously differentiable manifold (which, in fact, eliminates the edges) and, on the other hand, that the part $\Sigma_{1}$ contains at least three noncollinear points. We need then to specify the functional framework within which the problem must be formulated.

(1) The displacements $X_{i}$ are assumed ${ }^{2}$ to belong to $H^{2}(S)$. It follows that on $\partial S$ the displacements $X_{i}$ belong to $H^{3 / 2}(\partial S)$ and that the tangential rotation $\widetilde{\varphi}_{i}$

${ }^{2}$ The notation used for the Sobolev spaces is the classical one. See, for example, Lions-Magenes [8] or Duvaut-Lions [1]. 
and the doubly normal unitary elongation $\varepsilon_{n n}$ belong to $H^{1 / 2}(\partial S)$. This defines the functional space in which the data $\overline{\bar{C}}$ on $\Sigma_{1}$ must be taken.

(2) The given external volume forces $f_{i}, C_{i j}, \Phi_{i j}$ will be taken in $L^{2}(S)$. The external forces given on $\Sigma_{2}$ will be, as far as $T_{i}$ is concerned, in the restriction to $\Sigma_{2}$ of $H^{-3 / 2}(\partial S)$, and as far as $\widetilde{m}_{i}$ and $n$ are concerned, in the restriction to $\Sigma_{2}$ of $H^{-1 / 2}(\partial S)$.

(3) It will be assumed that the coefficients of the quadratic form $\mathrm{w}(c)$ belong to $L^{\infty}(S)$, that is, that they are essentially bounded.

Under these conditions, we can state:

Theorem 5. If the data $\overline{\mathscr{C}}$ and $\overline{\mathscr{F}}$ belong to the functional spaces introduced above, there exists a unique displacement field that minimizes the potential energy $V\left(C^{\prime}\right)$ among all the kinematically admissible fields (defined by $X_{i}^{\prime} \in\left(H^{2}(S)\right)$ and satisfying the boundary conditions $\overline{\mathscr{C}}$ on $\Sigma_{1}$ ).

The proof, which we will not carry out here, has been developed ${ }^{3}$ by Duvaut [12]. It relies on a generalized Korn inequality that can be deduced very simply from the classical inequality according to which the norm defined in $\left(H^{2}(\Omega)\right)^{3}$ by the inner product

$$
\left(\left(\mathscr{C}_{i}^{(1)}, \mathscr{C}_{i}^{(2)}\right)\right)=\int_{S}\left(X_{i}^{(1)} X_{i}^{(2)}+\varepsilon_{i j}^{(1)} \varepsilon_{i j}^{(2)}+\eta_{i j}^{(1)} \eta_{i j}^{(2)}+\eta_{i j k}^{(1)} \eta_{i j k}^{(2)}\right) d v
$$

is equivalent to the classical norm on $\left(H^{2}(\Omega)\right)^{3}$, and moreover, from the fact that if $\overline{\mathscr{C}}=0$, there exists a constant $M$ such that $\mathrm{w}(C) \geq M((\mathscr{C}, \mathscr{b}))$.

6.4. Possible generalizations. When extending the methods described in [3] to construct in the classical case the constitutive laws of viscoelastic or elastic-perfectly plastic media, it is not difficult to formulate, in the framework of the small perturbation hypothesis, theories of viscoelasticity or plasticity for a description of a continuous medium by means of a second-order theory. It will suffice, for example, to add to the function $\mathrm{w}(c)$ a conveniently chosen dissipation function. We will content ourselves here with this simple remark.

\section{Constitutive laws of a hyperelastic medium in a second-gradient theory}

In the preceding section, we have shown how the construction of the proposed second-gradient theory leads quite naturally to the formulation of constitutive laws in the case where the medium sustained only small perturbations. It remains for us to show how to proceed in the case of finite strains. We will content ourselves with considering the elastic case.

\footnotetext{
${ }^{3}$ Only the case of isotropic media is considered in [12]. But by means of appropriate conditions, the extension to the general case is straightforward.
} 
As is well known in the classical theory, the essential point is to obtain a representation of the stresses after convected transport from the configuration under study to the reference configuration. For the sake of simplicity, we will assume this configuration to be defined by the coordinates $a_{\alpha}(\alpha=1,2,3)$ in the orthonormal Cartesian frame used to describe the system $S$. We will denote by $F_{i \alpha}$ the elements of the gradient matrix and by a superposed dot the material derivative. If $U_{i}$ denotes the velocity vector, we can write

$$
U_{i, \alpha}=U_{i, j} F_{j \alpha}=\dot{F}_{i \alpha},
$$

where a Greek index such as $\alpha$ placed after a comma indicates a derivative with respect to $a_{\alpha}$, while the function being differentiated is expressed in terms of the Lagrangian variables $a_{1}, a_{2}, a_{3}, t$.

If we denote by $L_{\alpha \beta}$ the Green-Lagrange strain tensor

$$
2 L_{\alpha \beta}=F_{i \alpha} F_{i \beta}-\delta_{\alpha \beta},
$$

we easily obtain the classical formula

$$
\dot{L}_{\alpha \beta}=D_{i j} F_{i \alpha} F_{j \beta}
$$

whose interpretation would become clearer when using curvilinear coordinates, since it would make manifest that the material derivative of the Green-Lagrange tensor is nothing but the result of the convected transport of the rate of strain tensor to the reference configuration.

We deduce from (79) that

$$
\dot{L}_{\alpha \beta, \gamma}=D_{i j}\left(F_{i \alpha} F_{j \beta}\right)_{, \gamma}+D_{i j, k} F_{i \alpha} F_{j \beta} F_{k \gamma},
$$

which shows that the derivatives $D_{i j, k}$ (or, what amounts to the same, the $U_{i, j k}$ ) can be calculated by means of the gradient of $\dot{L}_{\alpha \beta}$. To utilize the representation of the second gradient that we have chosen, it is convenient to introduce the quantities

$$
\Lambda_{\alpha \beta \gamma}=\frac{1}{3}\left(L_{\alpha \beta, \gamma}+L_{\beta \gamma, \alpha}+L_{\gamma \alpha, \beta}\right), \quad \Lambda_{\alpha \beta}=-2 \varepsilon_{\alpha \rho \sigma} L_{\beta \rho, \sigma} .
$$

It should be noted that $\Lambda_{\alpha \beta \gamma}$ is invariant under any permutation of the indices $\alpha, \beta, \gamma$, and that $\Lambda_{\alpha \alpha}=0$. Indeed,

$$
2 \varepsilon_{\alpha \rho \sigma} L_{\beta \rho, \sigma}=\varepsilon_{\alpha \rho \sigma}\left(F_{i \beta} F_{i \rho}\right)_{, \sigma}=\varepsilon_{\alpha \rho \sigma} F_{i \beta, \sigma} F_{i \rho},
$$


since $F_{i \rho, \sigma}=\frac{\partial^{2} x_{i}}{\partial a_{\rho} \partial a_{\sigma}}$ is symmetric in $\rho$ and $\sigma$. For the same reason, $\varepsilon_{\alpha \rho \sigma} L_{\alpha \rho, \sigma}=0$. We have, therefore, in the first place

$$
\begin{aligned}
3 \dot{\Lambda}_{\alpha \beta \gamma}= & \dot{L}_{\alpha \beta, \gamma}+\dot{L}_{\beta \gamma, \alpha}+\dot{L}_{\gamma \alpha, \beta} \\
= & D_{i j}\left[\left(F_{i \alpha} F_{j \beta}\right)_{, \gamma}+\left(F_{i \beta} F_{j \gamma}\right)_{, \alpha}+\left(F_{i \gamma} F_{j \alpha}\right)_{, \beta}\right] \\
& \quad+D_{i j, k}\left[F_{i \alpha} F_{j \beta} F_{k \gamma}+F_{i \beta} F_{j \gamma} F_{k \alpha}+F_{i \gamma} F_{j \alpha} F_{k \beta}\right],
\end{aligned}
$$

or

$$
\dot{\Lambda}_{\alpha \beta \gamma}=P_{\alpha \beta \gamma i j} D_{i j}+K_{i j k} F_{i \alpha} F_{j \beta} F_{k \gamma} .
$$

Here, $K_{i j k}$ denotes the completely skew-symmetric part of $U_{i, j k}$, defined in (27), and $P_{\alpha \beta \gamma i j}$ are coefficients which remain invariant under permutations of the indices $\alpha, \beta, \gamma$, on the one hand, and the indices $i, j$, on the other. In fact, we may state

$$
P_{\alpha \beta \gamma i j}=\frac{1}{6}\left\{\left(F_{i \alpha} F_{j \beta}+F_{j \alpha} F_{i \beta}\right)_{, \gamma}+\left(F_{i \beta} F_{j \gamma}+F_{j \beta} F_{i \gamma}\right)_{, \alpha}+\left(F_{i \gamma} F_{j \alpha}+F_{i \alpha} F_{j \gamma}\right)_{, \beta}\right\} .
$$

Accordingly, we calculate

$$
\begin{aligned}
\dot{\Lambda}_{\alpha \beta} & =-2 \varepsilon_{\alpha \rho \sigma} \dot{L}_{\beta \rho, \sigma} \\
& =-2\left[\varepsilon_{\alpha \rho \sigma} D_{i j}\left(F_{i \beta} F_{j \rho}\right)_{, \sigma}+\varepsilon_{\alpha \rho \sigma} D_{i j, k} F_{i \beta} F_{j \rho} F_{k \sigma}\right] .
\end{aligned}
$$

It proves convenient to introduce the coefficients

$$
Q_{\alpha \beta i j}=-\varepsilon_{\alpha \rho \sigma}\left[\left(F_{i \beta} F_{j \rho}\right)_{, \sigma}+\left(F_{j \beta} F_{i \rho}\right)_{, \sigma}\right]=-\varepsilon_{\alpha \rho \sigma}\left[F_{i \beta, \sigma} F_{j \rho}+F_{j \beta, \sigma} F_{i \rho}\right] .
$$

The last equality is a result of the symmetry of $F_{j \rho, \sigma}$ with respect to the indices $\rho$ and $\sigma$. It should be noted, moreover, that

$$
\varepsilon_{\alpha \rho \sigma}\left[D_{i j, k} F_{j \rho} F_{k \sigma}+D_{i k, j} F_{j \rho} F_{k \sigma}\right]=\varepsilon_{\alpha \rho \sigma}\left[F_{j \rho} F_{k \sigma}\right]\left[D_{i j, k}+D_{i k, j}\right]=0,
$$

so that in (83) we can replace $D_{i j, k}$ by

$$
\frac{1}{2}\left(D_{i j, k}-D_{i k, j}\right)=\frac{1}{2} \Omega_{j k, i}=-\frac{1}{2} \varepsilon_{j k p} \omega_{p, i}=-\frac{1}{2} \varepsilon_{p j k} K_{p i},
$$

where the last equalities stem from (25) and (26).

Thus, (83) can be written as

$$
\dot{\Lambda}_{\alpha \beta}=Q_{\alpha \beta i j} D_{i j}+\varepsilon_{p j k} \varepsilon_{\alpha \rho \sigma} F_{i \beta} F_{j \rho} F_{k \sigma} K_{p i} .
$$

It should be noted that the right-hand side of (85) is actually a deviator. Indeed, $Q_{\alpha \alpha i j}=0$, since $\varepsilon_{\alpha \rho \sigma} F_{i \alpha, \sigma} F_{j \rho}=0$ by virtue of the symmetry of $F_{i \alpha, \sigma}$ in $\alpha$ and $\sigma$. Furthermore, if we denote, according to common usage,

$$
J=\operatorname{det}\left(F_{i \alpha}\right),
$$


we obtain

$$
\varepsilon_{p j k} \varepsilon_{\alpha \rho \sigma} F_{i \alpha} F_{j \rho} F_{k \sigma} K_{p i}=J \varepsilon_{p j k} \varepsilon_{i j k} K_{p i}=2 J K_{i i}=0,
$$

since $K_{i j}$ is a deviator.

These preliminary computations having been carried out, if we denote by $\rho_{0}$ the mass per unit volume in the reference state and by $\rho$ the mass per unit volume in the present state, it is clear that the energy per unit mass of the internal forces will be linear with respect to $L_{\alpha \beta}, \Lambda_{\alpha \beta}, D_{\alpha \beta \gamma}$. We thus write

$$
\frac{1}{\rho}\left(\sigma_{i j} D_{i j}+\mu_{i j} K_{i j}+\mu_{i j k} K_{i j k}\right)=\frac{1}{\rho_{0}}\left(s_{\alpha \beta} \dot{L}_{\alpha \beta}+\Pi_{\alpha \beta} \dot{\Lambda}_{\alpha \beta}+\Pi_{\alpha \beta \gamma} \dot{\Lambda}_{\alpha \beta \gamma}\right) .
$$

In this equation, we can assume, without loss of generality, that $s_{\alpha \beta}$ is symmetric, that $\Pi_{\alpha \beta}$ is a deviator, and that $\Pi_{\alpha \beta \gamma}$ is completely skew-symmetric in $\alpha, \beta, \gamma$. The tensors $s_{\alpha \beta}, \Pi_{\alpha \beta}, \Pi_{\alpha \beta \gamma}$ provide a representation of the intrinsic stresses in the reference configuration for a medium described in a second-gradient theory. Naturally, $s_{\alpha \beta}$ is the classical symmetric Piola-Kirchhoff tensor.

Using (79), (81), (85), (86), we can write (87) in the form

$$
\begin{aligned}
D_{i j}\left[J \sigma_{i j}-F_{i \alpha} F_{j \beta} s_{\alpha \beta}-Q_{\alpha \beta i j} \Pi_{\alpha \beta}\right. & \left.-P_{\alpha \beta \gamma i j} \Pi_{\alpha \beta \gamma}\right] \\
& +K_{i j}\left(J \mu_{i j}-\varepsilon_{i p q} \varepsilon_{\alpha \rho \sigma} F_{j \beta} F_{p \rho} F_{q \sigma} \Pi_{\alpha \beta}\right) \\
& +K_{i j k}\left(J \mu_{i j k}-F_{i \alpha} F_{j \beta} F_{k \gamma} \Pi_{\alpha \beta \gamma}\right)=0 .
\end{aligned}
$$

Since, by hypothesis, we are assuming that the medium is not subjected to any internal constraints, $D_{i j}, K_{i j}$, and $K_{i j k}$ can take arbitrary values. Moreover, the coefficient of $D_{i j}$ is symmetric in $i, j$; the coefficient of $K_{i j}$ represents the components of a deviator (since $\varepsilon_{\alpha \rho \sigma} \varepsilon_{\beta \rho \sigma} \Pi_{\alpha \beta}=0$ ); and the coefficient of $K_{i j k}$ is completely skew-symmetric in $i, j, k$. We obtain, therefore, that

$$
\left\{\begin{aligned}
J \sigma_{i j} & =F_{i \alpha} F_{j \beta} s_{\alpha \beta}+Q_{\alpha \beta i j} \Pi_{\alpha \beta}+P_{\alpha \beta \gamma i j} \Pi_{\alpha \beta \gamma}, \\
J \mu_{i j} & =\varepsilon_{i p q} \varepsilon_{\alpha \rho \sigma} F_{j \beta} F_{p \rho} F_{q \sigma} \Pi_{\alpha \beta}, \\
J \mu_{i j k} & =F_{i \alpha} F_{j \beta} F_{k \gamma} \Pi_{\alpha \beta \gamma},
\end{aligned}\right.
$$

identically. These equations express the intrinsic stress tensors in the present configuration as functions of the intrinsic stress tensors in the reference configuration. It is easy, moreover, to solve them in terms of $\Pi_{\alpha \beta \gamma}, \Pi_{\alpha \beta}, s_{\alpha \beta}$.

Let us assume that the medium is elastic and, more precisely, hyperelastic. The classical theory can be easily generalized (see, for example, Germain [3]) supposing that the specific free energy $\Psi$ is a function of the absolute temperature and of the variables $L_{\alpha \beta}, \Lambda_{\alpha \beta}, \Lambda_{\alpha \beta \gamma}$, it being understood that $L_{\alpha \beta}$ and $\Lambda_{\alpha \beta \gamma}$ make their contributions symmetrically and that $\Psi$ is invariant under the transformation $\Lambda_{\alpha \beta} \rightarrow \Lambda_{\alpha \beta}+C \delta_{\alpha \beta}$ for arbitrary values of the constant $C$. The differential of $\Psi$, 
while the temperature is kept constant, namely,

$$
\rho_{0}^{-1}\left(s_{\alpha \beta} d L_{\alpha \beta}+\Pi_{\alpha \beta} d \Lambda_{\alpha \beta}+\Pi_{\alpha \beta \gamma} d \Lambda_{\alpha \beta \gamma}\right),
$$

is identical to minus the infinitesimal work of the internal forces, so that we can write

$$
s_{\alpha \beta}=\rho_{0} \frac{\partial \Psi}{\partial L_{\alpha \beta}}, \quad \Pi_{\alpha \beta}=\rho_{0} \frac{\partial \Psi}{\partial \Lambda_{\alpha \beta}}, \quad \Pi_{\alpha \beta \gamma}=\rho_{0} \frac{\partial \Psi}{\partial \Lambda_{\alpha \beta \gamma}},
$$

which provides a simple first way to write the constitutive laws. Equations (89) lead directly to the expression of the stresses in the present configuration as

$$
\left\{\begin{aligned}
\sigma_{i j} & =\rho\left[\frac{\partial \Psi}{\partial L_{\alpha \beta}} F_{i \alpha} F_{j \beta}+\frac{\partial \Psi}{\partial \Lambda_{\alpha \beta}} Q_{\alpha \beta i j}+\frac{\partial \Psi}{\partial \Lambda_{\alpha \beta \gamma}} P_{\alpha \beta \gamma i j}\right], \\
\mu_{i j} & =\rho \varepsilon_{i p q} \varepsilon_{\alpha \rho \sigma} F_{j \beta} F_{p \rho} F_{q \sigma} \frac{\partial \Psi}{\partial \Lambda_{\alpha \beta}}, \\
\mu_{i j k} & =\rho \frac{\partial \Psi}{\partial \Lambda_{\alpha \beta \gamma}} F_{i \alpha} F_{j \beta} F_{k \gamma} .
\end{aligned}\right.
$$

Recall that the coefficients $P_{\alpha \beta \gamma i j}$ and $Q_{\alpha \beta i j}$ are expressed directly in terms of the gradient matrix and its derivatives according to (82) and (84).

It is to be noted that, under the hypothesis of small strains, we may use in (91)

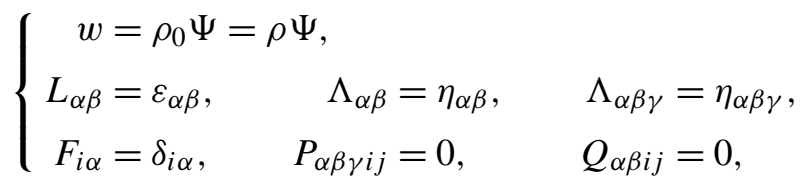

so that we still recover (59).

We have obtained, as we intended, the constitutive laws of a hyperelastic medium under finite strains in the framework of the second-gradient theory.

\section{Conclusion}

The aim of this first article - which develops a part of the results announced in a recent note [9] - has been to show how the method of virtual power provides a means at the same time powerful and natural to construct a theory of continuous media. The main results that emerge are the following:

(a) Construction of a first-gradient theory that offers itself as a very simple generalization of the classical theory and that exposes the distinction between the classical stress tensor and the intrinsic stress tensor (Theorem 1).

(b) Construction of a second-gradient-theory that generalizes the preceding one (Theorem 2). The fundamental formulas, which were already known in the case of elastic media, have a general scope. This appears to be a new result. 
(c) Statement of the main results of the second-gradient theory in the case of elastic media for infinitesimal strains. These results, without being essentially new, are collected in a systematic presentation.

Incidentally, a new introduction is put forward of the notion of screw (torsor) that perhaps better highlights its mechanical meaning. It was introduced by the author in an unpublished course [11]. See also [3] and [10].

Finally, we would like to remark that, in addition to the advantage of introducing an exact representation of the internal forces suited to the adopted description, this method allows us to obtain as naturally as possible and without new computations the notions of strain energy and the variational formulations that derive from it in the case where the medium is elastic. This notion is, more generally, perfectly adapted to the application of the principles of the thermodynamics of continuous media to obtain the constitutive laws of nonelastic media. We can also say that, without any new effort, this method permits to extend the results obtained in elasticity to the most general media by means of any formulation that takes as its point of departure a variational or Hamiltonian formulation.

\section{Appendix}

A.1. Preliminary formulas. It will be useful to introduce at each point of the surface $\partial \mathscr{D}$, the boundary of a connected domain $\mathscr{D}$, the operators of normal and tangential differentiation. Let $\varphi\left(x_{1}, x_{2}, x_{3}\right)$ be a continuous and continuously differentiable scalar-valued function defined on the closure $\mathscr{D}+\partial \mathscr{D}$ of $\mathscr{D}$. Its normal derivative (toward the exterior) is a scalar denoted by $D \varphi$ and its tangential derivative on the surface is the vector $D_{j} \varphi$ given by

$$
D \varphi=n_{l} \varphi_{, l}, \quad \varphi_{, j}=D_{j} \varphi+n_{j} D \varphi .
$$

We proceed in the same way with a vector-valued function $q_{i}\left(x_{1}, x_{2}, x_{3}\right)$. The normal derivative is the vector $D q_{i}$ and the tangential derivative is the tensor $D_{j} q_{i}$ given by the following expressions:

$$
D q_{i}=n_{l} q_{i, l}, \quad q_{i, j}=D_{j} q_{i}+n_{j} D q_{i} .
$$

Recall the statement of the divergence theorem on a surface. Let $\Sigma$ be a closed area, with continuous tangent plane and curvature, traced over the surface $\partial \mathscr{D}$, and let $\tau_{i}$ be the unit vector tangent to the boundary $\partial \Sigma$ oriented in the direct sense around the normal $n_{i}$ to $\Sigma$. Finally, let $v_{i}$ denote the exterior unit normal to $\partial \Sigma$ lying on the tangent plane to $\Sigma$, so that

$$
v_{j}=\varepsilon_{j m l} \tau_{m} n_{l}
$$


We can write

$$
\int_{\Sigma} D_{j} q_{j} d a=\int_{\Sigma} n_{j} q_{j}\left(D_{l} n_{l}\right) d a+\int_{\partial \Sigma \curvearrowleft} v_{j} q_{j} d s .
$$

Note that $D_{l} n_{l}$ is twice the mean curvature. We can verify this formula by noticing, for example, ${ }^{4}$ that

$$
D_{j} q_{j}=\left(D_{l} n_{l}\right) n_{j} q_{j}+\varepsilon_{s r m} \varepsilon_{m l j} n_{s}\left(n_{l} q_{j}\right)_{, r} .
$$

The last term is then $\boldsymbol{n} \cdot \operatorname{rot}(\boldsymbol{n} \wedge \boldsymbol{q})$, and the identity results from Stokes' formula.

Suppose now that the boundary $\partial \mathscr{D}$ is a closed surface with piecewise continuous tangent and curvature, and denote by $\Gamma$ the "edges" of $\partial \mathscr{D}$, along which there is a discontinuity of the tangent plane. We can write, according to (A-4),

$$
\int_{\partial \mathscr{D}} D_{j} q_{j} d a=\int_{\partial \mathscr{D}}\left(D_{p} n_{p}\right) n_{j} q_{j} d a+\int_{\Gamma \curvearrowleft} \llbracket v_{j} q_{j} \rrbracket d s,
$$

where [I \ denotes the jump of the enclosed quantity. Another form is obtained by applying (A-3):

$$
\int_{\partial \mathscr{D}} D_{j} q_{j} d a=\int_{\partial \mathscr{D}}\left(D_{p} n_{p}\right) n_{j} q_{j} d a+\int_{\Gamma \curvearrowleft} \varepsilon_{j m p} \tau_{m} \llbracket n_{p} q_{j} \rrbracket d s .
$$

We will need, furthermore, a canonical decomposition of the velocity gradient tensor different from that given by (A-2) and involving tangential derivatives of the velocities, the tangential components of the rate of rotation vector, and the doubly normal (to $\partial \mathscr{D}$ ) component ${ }^{5} D_{\underline{n} \underline{n}}$ of the rate of strain tensor. To this end it is sufficient to express the normal derivative of the velocity vector. But

$$
\begin{aligned}
D U_{k} & =n_{p}\left(D_{k p}+\Omega_{k p}\right) \\
& =n_{p} \Omega_{k p}+\frac{1}{2} n_{p} U_{k, p}+\frac{1}{2} n_{p} U_{p, k}=n_{p} \Omega_{k p}+\frac{1}{2} D U_{k}+\frac{1}{2} n_{p} U_{p, k} .
\end{aligned}
$$

We have, therefore,

$$
\begin{aligned}
D U_{k} & =2 n_{p} \Omega_{k p}+n_{p} U_{p, k}=2 n_{p} \Omega_{k p}+n_{p}\left(D_{k} U_{p}+n_{k} D U_{p}\right) \\
& =2 n_{p} \Omega_{k p}+n_{p} D_{k} U_{p}+n_{k} n_{p} n_{q} U_{p, q},
\end{aligned}
$$

and since $\Omega_{p q} n_{p} n_{q}=0$, we obtain

$$
D U_{k}=2 n_{p} \Omega_{k p}+n_{p} D_{k} U_{p}+n_{k} D_{\underline{n} \underline{n}} .
$$

Applying (A-2), we obtain the desired formula as

$$
U_{i, j}=D_{j} U_{i}+n_{j} n_{p} D_{i} U_{p}+2 n_{j} n_{p} \Omega_{i p}+n_{i} n_{j} D_{\underline{n} \underline{n}} .
$$

${ }^{4}$ We extend the definition of $\boldsymbol{n}$ to a neighborhood of $\partial \mathscr{D}$ by parallel transport along the normal.

${ }^{5}$ A repeated underlined index implies no summation. Here $D_{\underline{n} n}=D_{p q} n_{p} n_{q}$. 
The vector $n_{p} \Omega_{i p}$ is obviously situated on the tangent plane to $\partial \mathscr{D}$; moreover,

$$
n_{p} \Omega_{i p}=-\varepsilon_{i p k} \omega_{k} n_{p}=-\varepsilon_{i p k} \widetilde{\omega}_{k} n_{p},
$$

where $\widetilde{\omega}_{k}$ denotes the tangential component of the vector $\omega_{k}$. We can also write (A-7) in the form

$$
U_{i, j}=D_{j} U_{i}+n_{j} n_{p} D_{i} U_{p}-2 n_{j} \varepsilon_{i p k} \widetilde{\omega}_{k} n_{p}+n_{i} n_{j} D_{\underline{n} \underline{n}} .
$$

A.2. Transformation of Equation (30). Let us start with the term arising from the triply contracted product of two tensors of order 3 . We have, taking into consideration the symmetry of $\mu_{i j k}$,

$$
\begin{aligned}
\mu_{i j k} K_{i j k}=\mu_{i j k} U_{i, j k} & =\left(\mu_{i j k} U_{i, j}\right)_{, k}-\mu_{i j k, k} U_{i, j} \\
& =\left(\mu_{i j k} U_{i, j}\right)_{, k}-\left(\mu_{i j k, k} U_{i}\right)_{, j}+\mu_{i j k, j k} U_{i},
\end{aligned}
$$

and a subsequent integration and application of the divergence theorem yield

$$
\int_{\mathscr{D}} \mu_{i j k} K_{i j k} d v=\int_{\mathscr{D}} \mu_{i j k, j k} U_{i} d v-\int_{\partial \mathscr{D}} \mu_{i j k, k} n_{j} U_{i} d a+\int_{\partial \mathscr{D}} \mu_{i j k} U_{i, j} n_{k} d a .
$$

It remains to transform the last integral. To this end, we write the integrand, using (A-8), as

$\mu_{i j k} U_{i, j} n_{k}=D_{j} U_{i}\left(\mu_{i j k} n_{k}+\mu_{j p k} n_{i} n_{p} n_{k}\right)-2 \varepsilon_{i k q} \mu_{j k p} n_{j} n_{p} n_{q} \widetilde{\omega}_{i}+\mu_{i j k} n_{i} n_{j} n_{k} D_{\underline{n} \underline{n}}$.

We now integrate by noting that the first term is of the form $D_{j} q_{j}$ and applying (A-6). Finally, we obtain

$$
\begin{aligned}
\int_{\mathscr{D}} \mu_{i j k} K_{i j k} d v= & \int_{\mathscr{D}} \mu_{i j k, j k} U_{i} d v \\
& -\int_{\partial \mathscr{D}}\left\{\mu_{i j k, k} n_{j}+\left(D_{j}-n_{j}\left(D_{p} n_{p}\right)\right)\left(\mu_{i j k} n_{k}+\mu_{j l k} n_{i} n_{l} n_{k}\right)\right\} U_{i} d a \\
& +\int_{\partial \mathscr{D}}\left(\mu_{i j k} n_{i} n_{j} n_{k} D_{\underline{n} \underline{n}}-2 \varepsilon_{i k q} \mu_{k j p} n_{j} n_{p} n_{q} \widetilde{\omega}_{i}\right) d a \\
& +\int_{\Gamma \curvearrowleft} \varepsilon_{j m q} \tau_{m} \llbracket \mu_{i j k} n_{k} n_{q}+\mu_{j p k} n_{i} n_{p} n_{q} n_{k} \rrbracket U_{i} d s .
\end{aligned}
$$

We proceed in a similar fashion with the term arising from the doubly contracted product of the tensors $\mu_{i j}$ and $K_{i j}$ :

$$
\begin{aligned}
\mu_{i j} K_{i j} & =-\frac{1}{2} \varepsilon_{i p q} U_{p, q j} \mu_{i j}=-\frac{1}{2}\left(\varepsilon_{i p q} \mu_{i j} U_{p, q}\right)_{, j}+\frac{1}{2} \varepsilon_{i p q} \mu_{i j, j} U_{p, q} \\
& =-\frac{1}{2}\left(\varepsilon_{i p q} \mu_{i j} U_{p, q}\right)_{, j}+\frac{1}{2}\left(\varepsilon_{i p q} \mu_{i j, j} U_{p}\right)_{, q}-\frac{1}{2} \varepsilon_{i p q} \mu_{i j, j q} U_{p} .
\end{aligned}
$$

Integrating, we obtain

$$
\begin{aligned}
& \int_{\mathscr{D}} \mu_{i j} K_{i j} d v \\
& \quad=\frac{1}{2} \varepsilon_{i p q}\left\{\int_{\mathscr{D}} \mu_{p j, j q} U_{i} d v-\int_{\partial \mathscr{D}} \mu_{p j, j} n_{q} U_{i} d a-\int_{\partial \mathscr{D}} \mu_{i j} n_{j} U_{p, q} d a\right\} .
\end{aligned}
$$


It is convenient to write the last integral in a more suitable form. Let us set

$$
A_{i}=\mu_{i j} n_{j}, \quad \tilde{A}_{i}=A_{i}-n_{i} A_{k} n_{k},
$$

so that $\tilde{A}_{i}$ denotes the tangential component of $A_{i}$ and that we can write the integrand as

$$
-\frac{1}{2} \varepsilon_{i p q} U_{p, q} A_{i}=\omega_{i} A_{i}=\widetilde{\omega}_{i} \tilde{A}_{i}+n_{j} n_{k} \omega_{j} A_{k}
$$

Moreover,

$$
\tilde{A}_{i}=\left(\delta_{i k}-n_{i} n_{k}\right) A_{k}=\mu_{i q} n_{q}-n_{i} \mu_{k q} n_{k} n_{q}=\mu_{i q} n_{q}-n_{i} \mu_{\underline{n} \underline{n}},
$$

where $\mu_{\underline{n} \underline{n}}$ denotes the doubly normal component of $\mu_{i j}$. Furthermore,

$$
\begin{aligned}
n_{j} n_{k} \omega_{j} A_{k} & =-\frac{1}{2} \varepsilon_{i p q} U_{p, q} A_{k} n_{k} n_{i}=-\frac{1}{2} \varepsilon_{i p q} A_{k} n_{k} n_{i} D_{q} U_{p} \\
& =-\frac{1}{2} D_{q}\left(\varepsilon_{i p q} A_{k} n_{k} n_{i} U_{p}\right)+\frac{1}{2} D_{q}\left(\varepsilon_{i p q} A_{k} n_{k} n_{i}\right) U_{p} \\
& =-\frac{1}{2} D_{j}\left(\varepsilon_{i p j} n_{i} \mu_{\underline{n n}} U_{p}\right)+\frac{1}{2} D_{j}\left(\varepsilon_{i p j} n_{i} \mu_{\underline{n n}}\right) U_{p} .
\end{aligned}
$$

Invoking (A-5), we can write

$$
\begin{aligned}
\int_{\partial \mathscr{D}} n_{j} n_{k} \omega_{j} A_{k} d a & =\frac{1}{2} \int_{\mathscr{D}} D_{j}\left(\varepsilon_{i p j} n_{i} \mu_{\underline{n} \underline{n}}\right) U_{p} d a-\frac{1}{2} \int_{\Gamma \curvearrowleft} \llbracket v_{j} \varepsilon_{p i j} \mu_{\underline{n} \underline{n}} n_{p} \rrbracket U_{i} d s \\
& =\frac{1}{2} \int_{\partial \mathscr{D}} D_{j}\left(\varepsilon_{p i j} n_{p} \mu_{\underline{n} \underline{n}}\right) U_{i} d a+\frac{1}{2} \int_{\Gamma \curvearrowleft} \llbracket \mu_{\underline{n} \underline{n}} \rrbracket \tau_{i} U_{i} d s .
\end{aligned}
$$

Finally, collecting the various partial results, we obtain

$$
\begin{aligned}
\int_{\mathscr{D}} \mu_{i j} K_{i j} d v=\frac{1}{2} \int_{\mathscr{D}} & \varepsilon_{i p q} \mu_{p j, j q} U_{i} d v \\
& +\frac{1}{2} \int_{\partial \mathscr{D}}\left\{\varepsilon_{p i j} D_{j}\left(\mu_{\underline{n} \underline{n}} n_{p}\right)-\varepsilon_{i p q} \mu_{p j, j} n_{q}\right\} U_{i} d a \\
& +\int_{\partial \mathscr{D}}\left(\mu_{i q} n_{q}-n_{i} \mu_{\underline{n} \underline{n}}\right) \widetilde{\omega}_{i} d a+\frac{1}{2} \int_{\Gamma \curvearrowleft} \llbracket \mu_{\underline{n} \underline{n}} \rrbracket \tau_{i} U_{i} d s .
\end{aligned}
$$

Note that we can write

$$
\varepsilon_{p i j} D_{j}\left(\mu_{\underline{n} \underline{n}} n_{p}\right)=\varepsilon_{p i j} n_{p} \mu_{\underline{n} \underline{n}, j}
$$

since $D_{j} n_{p}=D_{p} n_{j}$. This permits us to give a different form to the surface integral that appears on the right-hand side of (A-11).

\section{References}

[1] G. Duvaut and J.-L. Lions, Les inéquations en mécanique et en physique, Travaux et Recherches Mathématiques 21, Dunod, Paris, 1972.

[2] R. D. Mindlin and N. N. Eshel, "On first strain-gradient theories in linear elasticity", Int. J. Solids Struct. 4:1 (1968), 109-124.

[3] P. Germain, Cours de mécanique des milieux continus, tome I: Théorie générale, ch. VII and VIII, Masson, Paris, 1973. 
[4] D. D. Lobdell, The role of nonsymmetric stress tensors and body torques in electromechanical phenomena in solids, Ph.D. thesis, Stanford University, 1970.

[5] R. D. Mindlin, "Micro-structure in linear elasticity", Arch. Rational Mech. Anal. 16 (1964), 51-78.

[6] R. A. Toupin, "Elastic materials with couple-stresses", Arch. Rational Mech. Anal. 11 (1962), $385-414$.

[7] P. Germain, Mécanique des milieux continus, tome 1, ch. VIII, Masson, Paris, 1962.

[8] J.-L. Lions and E. Magenes, Problèmes aux limites non homogènes et applications, vol. 1, Travaux et Recherches Mathématiques 17, Dunod, Paris, 1968.

[9] P. Germain, "Sur l'application de la méthode des puissances virtuelles en mécanique des milieux continus", C. R. Acad. Sci. A 274 (1972), 1051-1055.

[10] Y. Bamberger and J.-P. Bourguignon, "Torseurs sur un espace affine", technical report M25.0470, Centre de Mathématique de l'École Polytechnique, 1970.

[11] P. Germain, "Classical mechanics”, course notes, Stanford University, 1969.

[12] G. Duvaut, "Élasticité linéaire avec couples de constraintes: théorèmes d'existence", J. Mécanique 9 (1970), 325-333.

[13] P. Casal, "Capillarité interne en mécanique des milieux continus", C. R. Acad. Sci. 256 (1963), $3820-3822$.

[14] P. Casal, "La capillarité interne", Cahier du Groupe Français d'études de Rhéologie VI:3 (1961), 31-37.

[15] P. Casal, "La théorie du second gradient et la capillarité", C. R. Acad. Sci. A 274 (1972), 15711574.

[16] M. Epstein and R. E. Smelser, "An appreciation and analysis of Paul Germain's 'The method of virtual power in the mechanics of continuous media, I: Second-gradient theory',"', Math. Mech. Complex Systems 8:2 (2020), 191-199.

Received 16 Dec 2018. Revised 25 Jun 2019. Accepted 17 Aug 2019.

Paul Germain: Institut de Mécanique théorique et appliquée, Université de Paris VI, Paris, France

Deceased 26 Feb 2009 


\title{
AN APPRECIATION AND DISCUSSION OF PAUL GERMAIN'S "THE METHOD OF VIRTUAL POWER IN THE MECHANICS OF CONTINUOUS MEDIA I: SECOND-GRADIENT THEORY"
}

\author{
Marcelo Epstein AND Ronald E. SMElser
}

\begin{abstract}
Paul Germain's 1973 article on the method of virtual power in continuum mechanics has had an enormous impact on the modern development of the discipline. In this article we examine the historical context of the ideas it contains and discuss their continuing importance. Our English translation of the French original appears elsewhere in this volume (MEMOCS 8:2 (2020), 153-190).
\end{abstract}

\section{Introduction}

Among the many contributions of Paul Germain (1920-2009) to mechanics, this classical 1973 article [1973a] on the method of virtual power in continuum mechanics stands out for its enormous impact on the modern development of the discipline, as evidenced by hundreds of citations and by its direct or indirect influence in establishing a paradigm of thought for succeeding generations. In this article we examine the historical antecedents of the ideas contained in the article and discuss their continuing relevance.

The article was published in French in the Journal de Mécanique. It was soon followed by a sequel [Germain 1973b], written in English and dealing with media possessing microstructure. To the best of our knowledge, the original paper had not previously been translated into English, a gap we have sought to remedy by providing our own translation in this issue [Germain 2020].

This note is organized around three of the key ideas found in Germain's article: torsors, the rigidification axiom, and duality. We conclude with a brief discussion of the language in the paper and some of our terminological choices made in its translation.

\section{Communicated by Pierre Seppecher.}

MSC2010: primary 74A05, 74A10, 74A30; secondary 74B20.

Keywords: virtual power, gradient elasticity, duality, screws, functional analysis. 


\section{Screws or torsors}

It may be hard to believe these days, when a standard first-year course in engineering statics counts couples along with forces as fundamental pillars of the discipline, that the very notion of a couple was not introduced formally or otherwise until the year 1803. It was the genius of Louis Poinsot (1777-1859) that conceived, baptized, and formalized this idea at a young age in his Éleméns de Statique [Poinsot 1803] and extended it further to kinematical and dynamical theories in later works. ${ }^{1}$ Among Poinsot's important statements, we may mention the proof that every system of forces and couples in space can be reduced to a statically equivalent system consisting of a single resultant force through any given point and a single resultant couple. Moreover, a judicious choice of a particular line reduces the system to a force along it and a couple about it (that is, the couple can be represented as a pair of forces on a plane perpendicular to this line). In the English terminology, this contraption is known as a "wrench". Earlier, the Italian mathematician Giulio Giuseppe Mozzi (1730-1813) had proved [1763] that every rigid-body motion can be represented as a "twist", that is, a combination of a translation along a line and a rotation about this line, a result often attributed to Michel Chasles (1793-1880).

Although controversial at first, Poinsot's ideas slowly gained acceptance and were supported, expanded, and promulgated by figures such as Alfred Ferdinand Möbius (1790-1868), Julius Plücker (1801-1868), Felix Klein (1849-1925), Edward John Routh (1831-1907), Robert Stawell Ball (1840-1913), Eduard Study (1862-1930), and Richard von Mises (1883-1953), all of whom helped to generalize the original concept in various physical and mathematical directions. It may have been Plücker who first proposed to consider a single hybrid entity encompassing forces and moments, an entity which he called "dyname", a six-dimensional vector whose first three components represent the force, while the last three represent the couple. ${ }^{2}$ With the structure of $\mathbb{R}^{3}$ in the background, certain additional peculiar operations can be defined, inspired clearly by the original idea in statics. This concept entered the English language as "screw", a term used by Ball in the title of his original treatise [1900] dedicated exclusively to this topic. ${ }^{3}$ In his monumental five-volume treatise on rational mechanics, Paul Émile Appell (1855-1930),

\footnotetext{
${ }^{1}$ A delightful historical account of Poinsot and his times is given in [Grattan-Guinness 2014].

${ }^{2}$ Plücker introduced early on the concept of six coordinates (only four of which are independent) to describe the collection of lines in space. See, e.g., [Plücker 1846]. His mature views on analytic geometry are collected in a later treatise, which has been translated into English [Plücker 1868]. The introductory chapter makes reference to the dyname as an example of his geometric system, although a dyname requires two extra parameters to convey the magnitudes of the force and the moment. An English summary by Plücker himself on his approach can be found in [Plücker 1865; 1866].

${ }^{3}$ Published earlier as [Ball 1876]. In extending the ideas of Plücker, von Mises created his Motorrechnung or "motor calculus". A screw is a motor represented by two collinear vectors.
} 
following Ball, translates it into French as "torseur" [Appell 1902]. The English term "torsor" is reserved for a more abstract concept in algebraic geometry.

Definition. Let $\mathbb{E}^{3}$ denote the Cartesian affine space of $\mathbb{R}^{3}$. A screw (or torsor, or motor) is a vector field $\boldsymbol{v}$ over $\mathbb{E}^{3}$ such that there exists a fixed skew-symmetric matrix $\boldsymbol{W} \in \mathbb{R}^{3} \times \mathbb{R}^{3}$ with the property

$$
\boldsymbol{v}_{q}=\boldsymbol{v}_{p}+\boldsymbol{W}(q-p) \text { for all } p, q \in \mathbb{E}^{3} .
$$

Given a screw, its core matrix $\boldsymbol{W}$ is unique, as can be easily proved by assuming the contrary and using the definition. In $\mathbb{R}^{3}$ with the standard orientation every skewsymmetric matrix $\boldsymbol{W}$ can be equivalently represented by a vector, say $\boldsymbol{w}$, such that for all vectors $\boldsymbol{u}$ the identity

$$
\boldsymbol{W} \boldsymbol{u}=\boldsymbol{w} \wedge \boldsymbol{u}
$$

is satisfied, where, following the French engineering tradition, a wedge denotes the usual cross product of vectors. ${ }^{4}$ Thus, Equation (1) can be replaced by

$$
\boldsymbol{v}_{q}=\boldsymbol{v}_{p}+\boldsymbol{w} \wedge(q-p) .
$$

The relation between the components $W_{i j}$ of $\boldsymbol{W}$ and the components $w_{k}$ of the core vector $\boldsymbol{w}$ is

$$
w_{k}=-\frac{1}{2} \varepsilon_{k i j} W_{i j},
$$

where $\varepsilon_{k i j}$ are the components of the Levi-Civita alternating symbol.

Equiprojectivity. As a vector field, a screw satisfies the property of equiprojectivity. Its name derives from the fact that, due to the skew-symmetry of $\boldsymbol{W},(1)$ implies that

$$
\left(\boldsymbol{v}_{q}-\boldsymbol{v}_{p}\right) \cdot(q-p)=0,
$$

where a dot is used for the ordinary Cartesian inner product. It follows that any two vectors $\boldsymbol{v}_{p}$ and $\boldsymbol{v}_{q}$ have the same projection on the line determined by their points of application, namely, $\boldsymbol{v}_{q} \cdot(q-p)=\boldsymbol{v}_{p} \cdot(q-p)$. The converse of this statement is known as the theorem of Delassus. ${ }^{5}$ It states that every equiprojective vector field is a screw. From the viewpoint of equiprojectivity, it is a straightforward matter to formulate a theory of screws in infinite-dimensional Hilbert spaces, a generalization that so far does not seem to have a direct bearing on continuum mechanics.

\footnotetext{
${ }^{4}$ In $\mathbb{R}^{3}$ there is a definite relation between the cross product of vectors and the Grassmann or wedge product of multivectors.

${ }^{5}$ Named after Étienne Delassus (1868-1926), a French mathematician who made important contributions to the theory of partial differential equations. The "theorem" appears in the first few pages of his book [Delassus 1913]. Delassus uses the terminology of fields of moments for screws and special fields for equiprojective fields, and then proves their equivalence. The book contains interesting contributions to the theory and applications of nonholonomic constraints.
} 
The elements of reduction of a screw at a point. It follows from the definition that to completely describe a screw it is sufficient to specify its core vector $\boldsymbol{w}$ and its value $\boldsymbol{v}_{p}$ at an arbitrary point $p$. Put differently, a screw is completely defined by the pair $\left(\boldsymbol{w}, \boldsymbol{v}_{p}\right)$, denoted also by $\{\mathcal{W}\}_{p}$, whose entries are the elements of reduction of the screw $\boldsymbol{v}$ at the point $p$. This observation should suffice to convince ourselves that the collection $\mathcal{W}$ of all possible screws constitutes a six-dimensional real vector space, where vector addition and multiplication by a scalar are defined in the obvious way. As such, we can define its dual space $\mathcal{W}^{*}$ consisting of all the scalar-valued linear operators (or forms) on $\mathcal{W}$. An element of reduction in the dual space will be denoted with square (rather than curly) brackets, such as $[\mathcal{T}]_{p}$.

The two fundamental examples. The first fundamental example of a screw space (or torsor space) in mechanics is the space of twists (or kinematic torsors, or distributors), namely, the space $\mathcal{C}$ of rigid-body velocity fields. Indeed, choosing any point $p \in \mathbb{E}^{3}$ such a field is represented by

$$
\boldsymbol{v}_{x}=\boldsymbol{v}_{p}+\boldsymbol{\omega} \wedge(x-p) \text { for all } x \in \mathbb{E}^{3},
$$

where $\omega$ is the angular velocity vector, which, incidentally, is the core vector of the twist.

The second fundamental example of relevance to mechanics is the space $\mathcal{T}$ of wrenches (or static torsors), each of whose elements is a field of moments of any system of forces and couples. We know, since Poinsot's pioneering work, that this field can be represented as

$$
\boldsymbol{m}_{x}=\boldsymbol{m}_{p}+\boldsymbol{f} \wedge(x-p),
$$

where $f$ is the force resultant of the system, which turns out to be the core of the wrench.

Duality and inner product. In a Lagrangian mechanics framework, generalized forces at a configuration are elements of the dual of the tangent space of the configuration manifold at the point representing the configuration. The evaluation of a force (a covector) on a tangent vector ("virtual velocity") is interpreted as a virtual power. According to this mental paradigm, therefore, the space of wrenches should be regarded as the dual space of the space of twists. If the space of twists were to be endowed with an inner product, each covector (a wrench) would be naturally identified with a vector (a twist), and the action of the former on the latter would consist of their inner product.

In the case of a rigid body, however, we know exactly what the natural inner product should be. Indeed, the virtual power $\mathcal{P}$ of a system of forces (defined, say, 
by $[\mathcal{T}]_{p}$ ) on a field of rigid-body virtual velocities (defined by $\{\mathcal{C}\}_{p}$ ) is given by

$$
\mathcal{P}=[\mathcal{T}]_{p} \cdot\{\mathcal{C}\}_{p}=\boldsymbol{f} \cdot \boldsymbol{v}_{p}+\boldsymbol{m}_{p} \cdot \boldsymbol{\omega} .
$$

On the right-hand side of this equation, we are using the ordinary dot product of $\mathbb{R}^{3}$, while the middle part of the equation introduces the desired inner product in the sixdimensional space of twists. We remark that this definition is consistent in the sense that, as can be easily verified, the result is independent of the point of reduction $p$ chosen. Note that the core of one screw acts on the field element of the other, and vice versa. This operation was formalized by von Mises, who call it a scalar product. He also introduced a generalized cross product, called the motor product, and discussed the physical meaning of both operations [von Mises 1924a]. ${ }^{6}$

\section{The rigidification axiom}

Another important concept found in Germain's work is the axiom of rigidification, which, just as the concept of a wrench, echoes back to older traditions. Its earliest manifestation is the principle of solidification usually attributed to the Flemish scientist Simon Stevin (circa 1548-1620). In general terms, this principle establishes that the state of equilibrium of a (deformable) body is not altered if any part of it is replaced by a rigid body of the same geometry. Without providing an explicit statement of the principle, Stevin used it in his work on the equilibrium of fluids at rest [1586]..$^{7}$ Clairaut, Euler, Poinsot, and other important scientists made use of this principle in their treatment of equilibrium of continuous media [Truesdell 1968]. ${ }^{8}$

Within the framework of the principle of virtual power, a crucial role is played by the axiom of rigidification in the following form. The virtual power of the internal forces vanishes for all rigid-body virtual velocity fields. When this axiom is attached to the principle of virtual power, the equations of equilibrium (or motion) of a continuum, including the balance of angular momentum, are obtained directly. An interesting parallel can be drawn between this axiom and Walter Noll's axiom of objectivity [1963] or the equivalent derivation of the laws of continuum mechanics from the invariance of an energy equation under superposed rigid-body motions [Green and Rivlin 1964].

Since the principle of virtual power postulates an identity, valid for all possible virtual velocity fields, ${ }^{9}$ all conclusions obtained from the application of this

${ }^{6} \mathrm{~A}$ follow-up paper appeared in the same volume, and has been translated into English [von Mises 1924b].

${ }^{7}$ For a thorough historical treatment, see [Casey 1992].

${ }^{8} \mathrm{~A}$ historical account in connection with virtual power can be found in [Capecchi 2012].

${ }^{9}$ The admissible virtual velocity fields in a particular theory are, naturally, restricted to a given functional space. Nevertheless, it is always assumed that this space contains the space of twists $\mathcal{C}$. 
principle are legitimate for the system under consideration. ${ }^{10}$ Consider, for example, the wrench $\left[\mathcal{T}_{\text {int }}\right]$ of the internal forces integrated over any subbody. Since, by the axiom of rigidification, the virtual power of this wrench must vanish identically for all twists, we conclude that $\left[\mathcal{T}_{\text {int }}\right]$ must vanish identically, whether the body be deformable or not. This fact is usually interpreted as a manifestation of Newton's third law (of action and reaction). The condition of equilibrium is obtained, therefore, as the vanishing of the wrench $\left[\mathcal{T}_{\text {ext }}\right]$ of the external forces.

\section{Duality}

The mystical, religious, and philosophical appeal of the notion of duality is as old as recorded history and need not be considered here. It is, however, interesting to note that Isaac Newton (1642-1727) himself devoted much of his creative energies to hermetic writings, including a translation of the so-called Emerald Tablet in which Newton finds that, "That which is below is like that which is above and that which is above is like that which is below to do the miracles of one only thing." 11 In short, the idea that there is some kind of automatic correspondence of concepts at two complementary levels of discourse entered more or less explicitly into a scientific (and prescientific) description of the universe. Already Archimedes (circa 287-212 BCE) envisioned the law of the lever as some kind of compensating effect between forces and virtual displacements to produce the vanishing of the "one only thing", which is virtual work.

In mathematical terms, a finite-dimensional vector space $U$ automatically implies the existence of a dual space $U^{*}$ of the same dimension, consisting of all the scalar-valued linear functions on $U$. Moreover, there exists a canonical isomorphism between the dual $\left(U^{*}\right)^{*}$ of $U^{*}$ and the original $U$, as can be shown without difficulty. We have, in fact, already considered above the example of the duality between the six-dimensional vector spaces of wrenches and twists mutually involved in the production of virtual power.

In the Lagrangian conception of classical mechanics, the configuration space of a system with a finite number of degrees of freedom is a finite-dimensional differentiable manifold $Q$. At each point $q \in Q$, namely at every configuration of the system, the tangent space $T_{q} \mathrm{Q}$ is a vector space, interpreted physically as the carrier of all possible virtual velocities $\boldsymbol{v}_{q}$ away from this configuration. A force at $q$ is, therefore, an element $f_{q}$ of the dual space $T_{q}^{*} Q$, that is, a real-valued

\footnotetext{
${ }^{10}$ See, for example, [Salençon 2016].

${ }^{11}$ Newton's manuscript, in his always refreshingly legible handwriting, is housed in King's College Library of Cambridge University and cataloged under the identifier Keynes MS. 28. It is also digitally available [Newton 2010]. Considering that the date of publication of the first edition of the Principia is 1687, it is rather interesting to remark that this and other alchemy-related manuscripts by Newton have been dated approximately to the decade of the 1680 s.
} 
linear function on $T_{q} \mathcal{Q}$. The evaluation $\boldsymbol{f}_{q}\left(\boldsymbol{v}_{q}\right)$ is, therefore, interpreted as the virtual power produced by the force on the velocity. The crux of Germain's article consists of the extension of these ideas to the infinite dimensional realm.

Following Segev [1986], if a continuous medium $\mathcal{B}$ is regarded as a differentiable manifold with boundary, its configuration space $Q$ can be regarded as the set of all $C^{p}$ embeddings in the physical space (for some $p \geq 1$ ). This set is known to sustain the structure of an infinite-dimensional Banach manifold. Its tangent space at a configuration $q \in Q$ is a Banach space $T_{q} Q$ representing the collection of all virtual velocity fields at $q$. Its dual space can, therefore, be interpreted as a generalized force in continuum mechanics, a concept that embraces both external forces and stresses in their full generality. To make his point crystal clear, however, Germain restricts the configuration manifold $Q$ by effectively identifying it with a Hilbert space $\mathcal{V}$, a Banach space whose norm is induced by an inner product. As explained by Germain, this assumption affords a description of forces and stresses completely analogous to the finite-dimensional counterpart, avoiding the important technicalities of measures and distributions.

\section{Some terminological remarks}

Quite apart from the ordinary stylistic difficulties involved in literary or scientific translation, Germain's paper offers an additional challenge even for those familiar with the French language. It arises from the theoretical framework within which the discipline of mechanics is taught in France and other European countries, a framework that involves not only terminological but also conceptual differences with the prevailing tradition in English-speaking countries. These and other matters of general interest pertinent to the background and its history were the motivation for this short article.

We turn now to some specific vocabulary choices:

- The mechanics literature in French (and other romance languages) uses the term effort to designate quite generally any mechanical interaction. Thus, an effort may be an external force or an internal stress. There is no such simple equivalent in English. In Germain's English abstract of the paper under consideration, the term "effort" was rendered as "strength". In his second paper, however, written this time in English, Germain occasionally uses the term "force", placing it between quotation marks. He never uses the term "strength". Our policy has been to use the word "force" for all occurrences of effort, since both the context and the presence of an adjective are sufficient to make the meaning clear.

- We have rendered "produit scalaire" as "inner product", to avoid any possible ambiguity. 
- The French term "déformation" is translated as "strain" and must not be confused with the English "deformation".

- The expression "lois de comportement" is rendered as "constitutive laws".

- Germain often uses the terms "energy" and "power" interchangeably. We have respected this slight inaccuracy.

\section{References}

[Appell 1902] P. Appell, Traité de mécanique rationnelle, tome I: Statique, dynamique du point, 2nd ed., Gauthier-Villars, Paris, 1902.

[Ball 1876] R. S. Ball, "The theory of screws: a study in the dynamics of a rigid body", Math. Ann. 9:4 (1876), 541-553.

[Ball 1900] R. S. Ball, A treatise on the theory of screws, Cambridge University, 1900.

[Capecchi 2012] D. Capecchi, History of virtual work laws: a history of mechanics prospective, Science Networks, Historical Studies 42, Springer, 2012.

[Casey 1992] J. Casey, "The principle of rigidification”, Arch. Hist. Exact Sci. 43:4 (1992), 329-383.

[Delassus 1913] E. Delassus, Leçons sur la dynamique des systèmes matériels, Hermann et Fils, Paris, 1913.

[Germain 1973a] P. Germain, "La méthode des puissances virtuelles en mécanique des milieux continus, I: Théorie du second gradient”, J. Mécanique 12:2 (1973), 235-274.

[Germain 1973b] P. Germain, "The method of virtual power in continuum mechanics, II: Microstructure", SIAM J. Appl. Math. 25:3 (1973), 556-575.

[Germain 2020] P. Germain, "he method of virtual power in the mechanics of continuous media, I: Second-gradient theory”, Math. Mech. Complex Systems 8:2 (2020), 153-190. Translated by M. Epstein and R. E. Smelser.

[Grattan-Guinness 2014] I. Grattan-Guinness, "From anomaly to fundament: Louis Poinsot's theories of the couple in mechanics", Historia Math. 41:1 (2014), 82-102.

[Green and Rivlin 1964] A. E. Green and R. S. Rivlin, "On Cauchy's equations of motion”, Z. Angew. Math. Phys. 15 (1964), 290-292.

[von Mises 1924a] R. von Mises, "Motorrechnung, ein neues Hilfsmittel der Mechanik", Z. Angew. Math. Mech. 4:2 (1924), 155-181.

[von Mises 1924b] R. von Mises, "Anwendungen der Motorrechnung”, Z. Angew. Math. Mech. 4:3 (1924), 193-213. A translation by D. H. Delphenich can be found at www.neo-classical-physics.info/ uploads/3/4/3/6/34363841/von_mises_-_motor_algebra_-_ii.pdf.

[Mozzi 1763] G. Mozzi, Discorso matematico sopra il rotamento momentaneo dei corpi, Campo, Napoli, 1763.

[Newton 2010] I. Newton, "Keynes MS. 28”, in The Chymistry of Isaac Newton, edited by W. R. Newman, Indiana University, 2010.

[Noll 1963] W. Noll, "La mécanique classique basée sur un axiome d'objectivité", pp. 47-56 in La Méthode axiomatique dans les mécaniques classiques et nouvelles, edited by A. Châtelet and J.-L. Destouches, Gauthier-Villars, Paris, 1963.

[Plücker 1846] J. Plücker, System der Geometrie des Raumes in neuer analytischer Behandlungsweise, insbesondere die Theorie der Flächen zweiter Ordnung und Classe enthaltend, Scheller, Düsseldorf, 1846. 
[Plücker 1865] J. Plücker, “On a new geometry of space”, Philos. T. R. Soc. Lond. 155 (1865), 725791.

[Plücker 1866] J. Plücker, "Fundamental views regarding mechanics", Philos. T. R. Soc. Lond. 156 (1866), 361-380.

[Plücker 1868] J. Plücker, Neue Geometrie des Raumes gegründet auf die Betrachtung der geraden Linie als Raumelement, Teubner, Leipzig, 1868. A translation by D. H. Delphenich can be found at http://www.neo-classical-physics.info/uploads/3/4/3/6/34363841/pluecker_-_a_new_geometry_of_ space.pdf.

[Poinsot 1803] L. Poinsot, Élémens de statique, Volland, Paris, 1803.

[Salençon 2016] J. Salençon, Mécanique des milieux continus, tome I: Concepts généraux, École Polytechnique, Paris, 2016. Translated in Handbook of continuum mechanics: general concepts, thermoelasticity, Springer, 2001.

[Segev 1986] R. Segev, "Forces and the existence of stresses in invariant continuum mechanics", $J$. Math. Phys. 27:1 (1986), 163-170.

[Stevin 1586] S. Stevin, De beghinselen des waterwichts, Plantijn, Leiden, 1586. Translated in "The elements of hydrostatics", pp. 375-483 in The principal works of Simon Stevin, vol. 1: General introduction, mechanics, edited by E. J. Dijksterhuis, Swets, Amsterdam, 1955.

[Truesdell 1968] C. Truesdell, Essays in the history of mechanics, Springer, 1968.

Received 16 Dec 2018. Revised 25 Jun 2019. Accepted 17 Aug 2019.

MARCELO EPSTEIN: mepstein@ucalgary.ca

Department of Mechanical and Manufacturing Engineering, University of Calgary, Calgary, Canada

RONALD E. SMELSER: rsmelser@uncc.edu

Mechanical Engineering and Engineering Science, University of North Carolina at Charlotte,

Charlotte, NC, United States 



\section{Guidelines for Authors}

Authors may submit manuscripts in PDF format on-line at the submission page.

Originality. Submission of a manuscript acknowledges that the manuscript is original and and is not, in whole or in part, published or under consideration for publication elsewhere. It is understood also that the manuscript will not be submitted elsewhere while under consideration for publication in this journal.

Language. Articles in MEMOCS are usually in English, but articles written in other languages are welcome.

Required items. A brief abstract of about 150 words or less must be included. It should be selfcontained and not make any reference to the bibliography. If the article is not in English, two versions of the abstract must be included, one in the language of the article and one in English. Also required are keywords and a Mathematics Subject Classification or a Physics and Astronomy Classification Scheme code for the article, and, for each author, postal address, affiliation (if appropriate), and email address if available. A home-page URL is optional.

Format. Authors are encouraged to use $\mathrm{IAT}_{\mathrm{E} X}$ and the standard amsart class, but submissions in other varieties of $\mathrm{T}_{\mathrm{E}} \mathrm{X}$, and exceptionally in other formats, are acceptable. Initial uploads should normally be in PDF format; after the refereeing process we will ask you to submit all source material.

References. Bibliographical references should be complete, including article titles and page ranges. All references in the bibliography should be cited in the text. The use of $\mathrm{BIBT}_{\mathrm{E}} \mathrm{X}$ is preferred but not required. Tags will be converted to the house format, however, for submission you may use the format of your choice. Links will be provided to all literature with known web locations and authors are encouraged to provide their own links in addition to those supplied in the editorial process.

Figures. Figures must be of publication quality. After acceptance, you will need to submit the original source files in vector graphics format for all diagrams in your manuscript: vector EPS or vector PDF files are the most useful.

Most drawing and graphing packages - Mathematica, Adobe Illustrator, Corel Draw, MATLAB, etc. - allow the user to save files in one of these formats. Make sure that what you are saving is vector graphics and not a bitmap. If you need help, please write to graphics@msp.org with as many details as you can about how your graphics were generated.

Bundle your figure files into a single archive (using zip, tar, rar or other format of your choice) and upload on the link you been provided at acceptance time. Each figure should be captioned and numbered so that it can float. Small figures occupying no more than three lines of vertical space can be kept in the text ("the curve looks like this:"). It is acceptable to submit a manuscript with all figures at the end, if their placement is specified in the text by means of comments such as "Place Figure 1 here". The same considerations apply to tables.

White Space. Forced line breaks or page breaks should not be inserted in the document. There is no point in your trying to optimize line and page breaks in the original manuscript. The manuscript will be reformatted to use the journal's preferred fonts and layout.

Proofs. Page proofs will be made available to authors (or to the designated corresponding author) at a Web site in PDF format. Failure to acknowledge the receipt of proofs or to return corrections within the requested deadline may cause publication to be postponed. 
Mathematics and Mechanics of Complex Systems vol. 8 no. 2

Genotype-dependent virus distribution and competition of 101 virus strains

Nikolai Bessonov, Gennady A. Bocharov, Cristina Leon, Vladimir Popov and Vitaly Volpert

Modeling the linear dynamics of continuous viscoelastic systems on their infinite-dimensional central subspace

Angelo Luongo and Francesco D'Annibale

The method of virtual power in the mechanics of continuous media, I: Second-gradient theory

\section{Paul Germain}

An appreciation and discussion of Paul Germain's "The method of virtual power in the mechanics of continuous media, I: Second-gradient theory"

Marcelo Epstein and Ronald E. Smelser

MEMOCS is a journal of the International Research Center for the Mathematics and Mechanics of Complex Systems at the Università dell' Aquila, Italy.

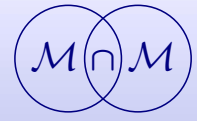

